\title{
The Impact of Geopolitical Risks on Travel and Leisure Stocks
}

\section{Sercan Demiralay}

The University of Sheffield International Faculty - CITY College

Department of Business Administration and Economics

Leontos Sofou 3

Thessaloniki 546 26, Greece

Email: sdemiralay@ citycollege.sheffield.eu

Erhan Kilincarslan

University of Huddersfield

Huddersfield Business School

Department of Accounting, Finance and Economics

Queensgate, HD1 3DH

Huddersfield, UK

Email: e.kilincarslan@hud.ac.uk

\section{Corresponding Author:}

Sercan Demiralay

Accepted on $2^{\text {rd }}$ June 2019 by Tourism Management - ABS 4* 


\title{
The Impact of Geopolitical Risks on Travel and Leisure Stocks
}

\begin{abstract}
The impact of geopolitical risks on tourism has not received scholarly attention in the existing literature. Previous studies focus on the effects of terrorism, which is a subset of geopolitical risks. Our paper fills this gap and contributes to the literature by analyzing the vulnerability of four regional Travel and Leisure (T\&L) industry stock indices to geopolitical risks. Employing traditional and quantile regression techniques, our results suggest a greater sensitivity of T\&L stock returns to geopolitical risks at times the industry performs poor, except for Asia \& Pacific index that is the most resilient to geopolitical events. We find that the negative effects of geopolitical risks are mostly driven by the threat of adverse geopolitical events only during period of falling T\&L stock prices. However, the realization of adverse geopolitical events significantly influences $T \& \mathrm{~L}$ stocks at all times. The results provide potential insights and implications for investors and tourism stakeholders.
\end{abstract}

Keywords: Geopolitical risks, travel and leisure industry, stock returns, quantile regressions

\section{Introduction}

Corporate managers, policy makers and media often identify geopolitical risks as one of the most important determinants of consumption and investment decisions. Caldara and Iacoviello (2018) define geopolitical risk as "the risk associated with wars, terrorist acts, and tensions between states that affect the normal and peaceful course of international relations." Accordingly, increased geopolitical uncertainty might lead to a delay in consumption and give firms an incentive to postpone investments due to the precautionary savings motive (Cheng and Chiu, 2018). In this respect, "Travel and Leisure (hereafter T\&L) industry" is particularly known as a highly fragile industry that is greatly vulnerable to external shocks such as war acts, terrorist attacks and nuclear threats - in other words, geopolitical risks (Ioannides and Apostolopoulos, 1999; Seetanah, 2011; Kim and Marcouiller, 2015; Seraphin, 2017). For instance, the 2015 Paris terror attacks showed how the tourism industry is susceptible to geopolitical actions since European travel and hotel companies' shares experienced a huge loss of more than $€ 2$ billion in total just on the day right after the attack (Wearden and Allen, 2015). Considering the recent dramatic events (such as the escalation of the Islamic States in Iraq and Syria (ISIS), Syrian civil war, Crimea annexation and nuclear threats from North Korea), we therefore argue that geopolitical threats are a new type of catastrophic risk that corporate managers, investors and authorities in the tourism industry have to face over the last several decades.

The vast majority of studies in tourism literature examine the relationship between terrorism and inbound/outbound tourism, or international tourism development (see, among others, Enders et al, 1992; Sonmez, 1998; Sonmez et al., 1999; Pizam and Smith, 2000; Drakos and Kutan, 2003; Arana and Leon, 2008; Sandler and Enders, 2008; Rittichainuwat and Chakraborty, 2009; Bassil, 2014; Buigut and Amendah, 2016; Liu and Pratt, 2017; Lanouar and Goaied, 2019). After the tragedy of the 9/11 attacks that had significant economic repercussions on tourism industry across the world, researcher have also focused on the impacts of terrorism 
on tourism-related companies' performance. However, there are relatively few studies that have been devoted to analyze the response of tourism companies' stock returns to terror events and political tensions (see, for example, Drakos, 2004; Kim and Gu, 2004; Madanoglu et al., 2007; Chen, 2011; Chang and Zeng, 2011; Zopiatis, 2018). Overall conclusion of these studies is that terrorism causes poor performance of T\&L stock returns.

Although numerous studies examine the effects of terrorist activities on tourism in general, the impact of geopolitical risks is largely ignored due to the lack of a time-varying indicator. Also, most of the relevant research focuses on the influence of a single terrorist event by using a dummy variable or utilizing an event study approach that has certain weaknesses. ${ }^{1}$ In a recent study, however, Caldara and Iacoviello (2018) develop a global geopolitical risk (hereafter GPR) index by counting the number of occurrences in articles of the leading international newspapers that discusses geopolitical tensions, risks and events. Their index does not only capture terrorist acts and threats but also considers war risk, nuclear threats and military-related tensions. Therefore, it is a broader measure and represents a wide array of exogenous global uncertainty (Balcilar et al., 2018).

This paper analyzes the effect of global geopolitical risks on Travel and Leisure (T\&L) companies' stock returns at regional level. To the best of our knowledge, this is the first paper in the literature to investigate the vulnerability of T\&L industry stock returns to geopolitical uncertainty. More specifically, our objective is to enhance our understanding of how T\&L stock indices (namely, STOXX T\&L Global, STOXX T\&L Asia-Pacific, STOXX T\&L Europe and STOXX T\&L North America) react to geopolitical risks. We employ both traditional and quantile regression (QR) techniques. The usage of classical linear regression models (CLRM) has always been the norm in tourism literature. This study moves beyond these traditional models and extends the relevant literature. As suggested by Assaf and Tsionas (2018), QR modelling approach has recently emerged as an attractive alternative to CLRM in tourismrelated research. Unlike the conventional mean models, it enables us to assess the responses of a dependent variable at different quantiles, providing evidence beyond the central location of the data. Therefore, this paper does not only analyze the average response but also measures the effect over the whole conditional distribution of the T\&L stock returns. In other words, we quantify the impact of geopolitical risks during different market states, such as episodes of falling T\&L stock returns (bear market), expected market conditions (normal market) and increasing T\&L stock returns (bull market).

Our paper attempts to address the following research questions: i) How vulnerable are T\&L stock returns to geopolitical risks? ii) Does the sensitivity of T\&L companies' stock performance to geopolitical risks change across varying market states? iii) Do geopolitical threats or acts drive T\&L stock returns more? After controlling for investor sentiment (measured by the volatility index (VIX)), oil prices and 2007-2009 global financial crisis, the overall empirical findings suggest vulnerability of T\&L stocks to geopolitical risks across all the markets, except for Asia\&Pacific being the most resilient to geopolitical events. Moreover, the results provide evidence of state dependent sensitivity of T\&L stocks to geopolitical tensions; the sensitivity is stronger in times $\mathrm{T} \& \mathrm{~L}$ industry performs poor. We further analyze the effects of geopolitical acts and threats using the two indices decomposed into threats and

\footnotetext{
${ }^{1}$ Event studies are known to be very sensitive to the choice of the window size, which may cast doubt on reliability of the empirical results.
} 
acts components by Caldara and Iacoviello (2018). The results reveal that the negative effects of geopolitical risks on T\&L stocks are mostly driven by the threat of adverse geopolitical events only during bearish market times. However, the acts significantly influence T\&L stocks under all market states even if they produce smaller effects during bearish times compared to threats. The robustness of the results is assessed by including a potentially important omitted control variable; namely, economic policy uncertainty. The empirical findings report similar results, showing evidence of consistency and confirming the robustness of the main findings. Our paper provides important insights and implications for stakeholders, travel and leisure stock investors, tourism managers and policy makers.

The rest of the paper is organized as follows. Section 2 presents the theoretical perspective. Section 3 explains the methodology and the data. Section 4 reports the empirical findings, whereas Section 5 discusses the potential implications. Section 6 concludes the paper.

\section{Theoretical Framework}

The analysis of stock price valuation can help us to understand how geopolitical risks affect T\&L stock prices. The price of a stock is determined by the future earnings (or dividend streams) and the discount rate (or rate of return required by stockholders) based on the standard dividend discount model. Since geopolitical uncertainty is an exogenous shock that hits the economies, fears and concerns about terror attacks, war risks and political uprisings lead to a downward revision of growth prospects for companies. The increased uncertainty lowers the dividend growth rate and raises the required return on equity investments. Mathematically, the fundamental stock pricing formula can be shown as below (Ross et al., 2008; Chen, 2011):

$P_{i, t}=\sum_{t=1}^{T} \frac{\operatorname{Div}_{t}}{(1+r)^{t}}$

where $\operatorname{Div}_{t}=\mathrm{p} x$ Net Income $t$

Net Income ${ }_{t}=\mathrm{EBIT}_{t}-\mathrm{I}_{t}-\mathrm{T}_{t}$

$\mathrm{EBIT}_{t}=\mathrm{R}_{t}-\mathrm{COS}_{t}-\mathrm{OE}_{t}$

In Equation (1), $\mathrm{P}_{i, t}$ is the price of the stock $i$ at time $t$; $\operatorname{Div}_{t}$ is the dividend generated by the stock; $r$ is the discount rate; EBIT $_{t}$ represents earnings before interest and taxes; $\mathrm{p}$ is the dividend payout ratio; $\mathrm{R}_{t}$ is the total revene that includes both sales and other types of revenue, $\operatorname{COS}_{t}$ denotes cost of sales; $\mathrm{I}_{t}$ is interest payments on the firm's debt; $\mathrm{T}_{t}$ stands for the tax payment and $\mathrm{OE}_{t}$ is the operational expenses. As can be seen, the pricing formula suggests that a stock price is determined by changes in corporate earnings and discount rate (or required rate of return). ${ }^{2}$

\footnotetext{
${ }^{2}$ In equation (1), we present a model of stock valuation where the price of a share of common stock to the investor is equal to the present value of all of the expected future dividends. It is possible to show stock pricing formula with different versions of valuation for different types of stocks such as stocks with constant dividend growth and with differential growth. In Gordon growth model, the value of a stock can be calculated as $\mathrm{P}_{i}=\mathrm{Div}_{1} /(\mathrm{r}-\mathrm{g})$, where $\mathrm{P}_{i}$ represents the price of the stock $i, \mathrm{D}_{1}$ is the next year's expected annual dividend per share, $\mathrm{g}$ denotes the expected dividend growth rate and $r$ is the investor's discount rate or required rate of return. The discount rate can be calculated from the Gordon model as $r=\left(\operatorname{Div}_{1} / P_{i}\right)+\mathrm{g}$. Note that the required return or discount rate represent cost of equity for companies and can also be estimated using the Capital Asset Pricing Model (CAPM).
} 
Heightened geopolitical risks can lead to a decline in the earnings of T\&L companies through two main channels. As illustrated by Equation (1), the first channel is the dividend channel. In an environment of heightened uncertainty due to geopolitical risks, individuals can be reluctant to travel abroad due to safety and security reasons (Dragouni et al., 2016; Demir and Gozgor, 2018). This results in a delay or cancelation of travel plans, drops in hotel occupancy and a decline in tourism demand (Enders and Sandler, 1991; Drakos and Kutan, 2003; Chen and Noriega, 2004). Furthermore, geopolitical risks may give rise to higher cost of doing business for tourism companies through increased security costs, higher insurance premiums and at-risk compensation costs (Kunreuther and Michel-Kerjan, 2004; Samatas, 2007). The decreased demand and increased costs are expected to lower company earnings in T\&L industry, which in turn leads to reduced dividends and stock prices.

The second channel is the discount rate channel. Total risk of an asset can be decomposed into two parts: systematic and idiosyncratic (Lintner, 1965). Geopolitical risks represent systematic risks that are unpredictable and non-diversifiable. Systematic risk is the only type of risks priced by rational investors in equilibrium since investors must be rewarded for bearing it (Drakos, 2004). Investors require higher return as compensation by changing their perceptions about the riskiness of T\&L stocks during the periods of high geopolitical uncertainty. Thus, geopolitical risks rise return required by $\mathrm{T} \& \mathrm{~L}$ stock investors (or discount rate), causing lower stock prices.

\section{Methodology and Data Analysis}

\subsection{Methodology}

We investigate the effects of geopolitical risks on T\&L stock returns based on the estimation of both classical linear regression models (CLRM) and quantile regression (QR) models. The CLRM model is a mean model that estimates the average response of dependent variable to explanatory variables. However, it only detects the central tendency of the data and thus does not allow any distinction between large and small stock price fluctuations or bull and bear markets. In this regard, the QR model gives a more comprehensive picture of the T\&L indices behavior as it allows the effect of each independent variable to change over the whole spectrum of the T\&L indices' return distributions. More specifically, the QR model enables us to have distinctions among bearish, normal and bullish market states. Furthermore, the QR model is more robust to heteroskedasticity, outliers and non-normality than the CLRM model (Brooks, 2013). Therefore, the QR functions provide more accurate results of the effects of regressors on the regressand (Koenker, 2005; Mensi et al., 2014).

We employ the CLRM to measure the average sensitivity of T\&L stock returns to geopolitical risks after controlling for other explanatory variables. The CLRM equation is specified as follows:

$r_{t}=\mu_{i}+\alpha y_{t}+\beta X_{t}+\varepsilon_{t}, \quad \varepsilon_{t} \sim\left(0, \sigma^{2}\right)$

where $\mathrm{r}_{t}$ represents the $\mathrm{T} \& \mathrm{~L}$ stock index return in month $t, \mathrm{y}_{t}$ is the change in geopolitical risk index in month $t, \varepsilon_{t}$ is the error term and $\mathrm{X}$ is a matrix of control variables. $\mu_{i}$ is the constant term, $\alpha$ and $\beta$ are the parameters to be estimated; the coefficient $\alpha$ captures the effects of geopolitical risks and the parameter $\beta$ measures the impact of the control variables. As mentioned before, we use the geopolitical risk index (GPR) constructed by Caldara and Iacoviello (2018). The GPR index is computed each month by counting the number of articles 
related to geopolitical risk in eleven newspaper as a share of the total number of news articles. ${ }^{3}$ Following the relevant literature, control variables consist of changes in CBOE Volatility index (VIX), oil prices and a dummy variable that takes value of 1 during the global financial crisis of 2007-2009 and 0 otherwise. ${ }^{4}$

In our study, we use the QR model to examine the effects of geopolitical risks, conditional on the various quantiles of the T\&L stock returns, capturing different market phases, such as bearish, normal and bullish market conditions. In the QR setting, we estimate the following model:

$Q_{r_{t}}\left(\tau \mid y_{t}, X_{t}\right)=\mu_{i}^{(\tau)}+\alpha^{(\tau)} y_{t}+\beta^{(\tau)} X_{t}+\varepsilon_{t}^{(\tau)}, \varepsilon_{t} \sim\left(0, \sigma^{2,(\tau)}\right)$

where $Q_{r_{\tau \& L, t}}\left(\tau \mid y_{t}, X_{t}\right)$ is the $\tau$ th quantile of the distribution of $\mathrm{r}_{t}$ given $\mathrm{y}$ and X. $\alpha^{(\tau)}$ determines the effect of GPR at the $\tau$ th conditional quantile of T\&L stock returns. The effect may be i) constant if $\alpha^{(\tau)}$ values do not change across quantiles, ii) increasing (decreasing) if $\alpha^{(\tau)}$ values increase (decrease) monotonically across quantiles and iii) asymmetric (symmetric) if $\alpha^{(\tau)}$ values are different (similar) for high and low quantiles. We estimate the quantile regressions at twenty different quantiles from 0.05 to 0.95 to have a broader perspective.

For a given $\tau$, the $\mathrm{QR}$ estimator solves the following problem:

$\hat{\beta}(\tau)=\underset{\beta \in R}{\arg \min } \sum_{t=1}^{T} \rho_{t}\left(r_{t}-\mu_{i}^{(\tau)}-\alpha^{(\tau)} y_{t}-\beta^{(\tau)} X_{t t}\right)$

where $\rho(\mathrm{z})=\mathrm{z}(\rho-\mathrm{I}(\mathrm{z}<0))$ is the check function and $I($.$) is an indicator function that takes$ value of 1 if $z \leq 0$ and 0 otherwise.

The CLRM regressions are performed with Heteroskedasticity and Autocorrelation Consistent (HAC) covariance matrix estimators to have a valid inference. Different diagnostic tests are applied to check the violation of the CLRM assumptions. The residual diagnostics are checked by the Durbin-Watson autocorrelation and White's heteroskedasticity tests. In QR estimations, the pairs bootstrapping procedure of Buchinsky (1995) is used to calculate standard errors as the errors computed with this method are asymptotically valid under misspecification and heteroscedasticity. ${ }^{5} \mathrm{We}$ also evaluate the validity of the quantile regressions based on different

\footnotetext{
${ }^{3}$ For constructing the indices, Caldara and Iacoviello (2018) code the sample of articles as GPR=1, GPR=0 and $\mathrm{GPR}=-1$, as follows: $1=$ the article has references to high or rising geopolitical risks. $0=$ the article has no references to geopolitical risks, or is uninformative whether geopolitical risks are rising or falling. $-1=$ the article has references to low or declining geopolitical risks. For more information about the construction of the GPR indices, visit https://www2.bc.edu/matteo-iacoviello/gpr.htm.

${ }^{4}$ The VIX, oil prices and financial crises are important determinants of outbound tourism, tourism development and hence tourism-related stock returns as recently found in Wang (2013), Dragouni et al. (2016) and Ersan et al. (2018). Since our study is at regional-level, it is not possible to include additional control variables as other macroeconomic or non-macroeconomic data are country-specific.
}

5 The Monte Carlo simulation results by Buchinsky (1995) show that the pairs-bootstrap estimator produces the best results. 
statistical tests, such as Quasi Likelihood ratio (QLR) and the sloe equality tests by Koenker and Bassett (1982), and further assess the goodness-of-fit by Pseudo R-squared. ${ }^{6}$

\subsection{Data analysis}

As stated earlier, our objective is to econometrically quantify the impact of geopolitical risks on travel and leisure stock indices. Specifically, four T\&L indices are selected from different regions - namely, STOXX Travel \& Leisure Global, STOXX Travel \& Leisure Asia-Pacific, STOXX Travel \& Leisure Europe and STOXX Travel \& Leisure North America. The data is retrieved from Bloomberg at monthly frequency.

Travel \& Leisure index is comprised of several different sub-industries and sectors, including airlines, gambling, hotels, recreational services, restaurants \& bars and travel \& tourism. It includes the vast majority of currently traded travel, leisure and hospitality companies' stocks around the globe, providing a broader perspective. It is worth noting that STOXX Travel \& Leisure indices categorize companies based on free-float market capitalization. Therefore, it includes the biggest T\&L companies across the globe and the region, such as Delta Airlines, Marriot International, Accor Hotels and Lufthansa Airlines. ${ }^{7}$ T\&L industry indices summarize tourism-related companies' stock performance and allow us to benchmark, analyze and compare the behavior of the industry. Using industry-level data is appropriate because it enables researchers and investors to quantitatively analyze the T\&L industry responses to events and compare among different regions (Zopiatis et al., 2018).

We use monthly T\&L stock indices from January 1992 to August 2018. ${ }^{8}$ We compute stock returns as the first difference of the natural $\log$ of each T\&L index. Table 1 presents the summary statistics and unit-root test results. ${ }^{9}$ The average return of each index is positive with North America having the highest value, followed by the global index. The unconditional risk, measured by standard deviations, is larger for Europe followed by North America. Skewness and kurtosis values suggest leptokurtic distribution of the index returns. The values of skewness are all negative, which indicates that having a larger negative return is more probable than having a larger positive return in all T\&L indices. The kurtosis values are greater than three, indicating a high likelihood of observing extreme negative values in the return distribution of

\footnotetext{
${ }^{6}$ It is worth noting that, as described by Koenker and Machado (1999), a goodness-of-fit statistic for quantile regression is analogous to the R-squared from traditional regression analysis. Pseudo R-squared for a given quantile measures the relative success of the model in fitting the data. Quasi Likelihood ratio (QLR) tests can be regarded as a variant of the F-test in CLRM with a joint hypothesis imposing restriction on regression coefficients. We did not include the test results in the paper to save space; however, all the test results show that the QR models fit the data and they are well-specified. For additional details about the testing procedures, see Koenker and Machado (1999).

7 The countries (and their weightings) included in STOXX Travel \& Leisure Global index are United States (60.2\%), Japan (17.3\%), Great Britain (9.3\%), Hong Kong (3.4\%), Australia (2.9\%), France (2.1\%), Ireland (1.7\%), Canada (1.2\%), Singapore (1.1\%) and Germany (0.4\%). The countries (and their weightings) in STOXX Travel \& Leisure Asia-Pacific index are Japan (66.6\%), Hong Kong (17.5\%), Australia (11.2\%), Singapore (4.1\%) and New Zealand $(0.6 \%)$. The countries (and their weightings) in STOXX Travel \& Leisure Europe index are France (55.2\%), Ireland (34.4\%) and Germany (13.4\%). The countries (and their weightings) in STOXX Travel $\&$ Leisure North America index are United States (98\%) and Canada (2\%).

${ }^{8}$ The selection of research period is based on data availability.

${ }^{9}$ For the sake of brevity, we do not report the descriptive statistics of the control variables and GPR index. The first difference of the log control variables is stationary and suitable for regression analyses. Test results are available upon request.
} 
the T\&L indices. The Augmented Dickey-Fuller (ADF) test results show the rejection of a unit root; therefore, $T \& \mathrm{~L}$ index returns are stationary and appropriate for further analysis.

Table 1. Summary Statistics of T\&L Index Returns

\begin{tabular}{lcccc}
\hline & Global & Asia-Pacific & Europe & North America \\
\hline Mean & 0.005099 & 0.001227 & 0.003531 & 0.007409 \\
Median & 0.010956 & 0.002713 & 0.010409 & 0.010312 \\
Maximum & 0.118183 & 0.180414 & 0.16812 & 0.16453 \\
Minimum & -0.211249 & -0.246468 & -0.21498 & -0.20152 \\
Std. Dev. & 0.044572 & 0.049426 & 0.054241 & 0.051044 \\
Skewness & -0.973764 & -0.449418 & -0.917925 & -0.677909 \\
Kurtosis & 5.65289 & 5.286058 & 5.082131 & 4.506613 \\
ADF & $-15.61491^{\mathrm{a}}$ & $-16.28627^{\mathrm{a}}$ & $-15.29543^{\mathrm{a}}$ & $-16.6515^{\mathrm{a}}$ \\
\hline
\end{tabular}

Notes. (a) represents the statistical significance at the $1 \%$ level. ADF is the Augmented Dickey-Fuller test for unit-roots.

The monthly geopolitical risk data is extracted from the work of Caldara and Iacoviello (2018). They construct a monthly index of Geopolitical Risk by counting the occurrence of words related to geopolitical tensions in eleven leading national and international newspapers. ${ }^{10}$ The index is constructed by searching articles including references to six groups of words. The authors explain their technique as follows: "Group 1 includes words associated with explicit mentions of geopolitical risk, as well as mentions of military-related tensions involving large regions of the world and a U.S. involvement. Group 2 includes words directly related to nuclear tensions. Groups 3 and 4 include mentions related to war threats and terrorist threats, respectively. Finally, Groups 5 and 6 aim at capturing press coverage of actual adverse geopolitical events (as opposed to just risks) which can be reasonably expected to lead to increases in geopolitical uncertainty, such as terrorist acts or the beginning of a war. The authors further disentangle the direct effect of adverse geopolitical events from the effect of pure geopolitical risks by constructing two indexes. The Geopolitical Threats (GPT) index only includes words belonging to Search Groups 1 to 4 above. The Geopolitical Acts (GPA) index only includes words belonging to Search Groups 5 and 6."

Figure 1 exhibits the GPR index during the study period. As the figure shows, the index tends to rise sharply during dramatic events, such as $9 / 11$ terror attack, the Iraq invasion, ISIS escalation and nuclear tests. Therefore, the GPR index is highly responsive to important political events and captures all significant geopolitical risk factors. In other words, the GPR index is a very good proxy for geopolitical risks.

\footnotetext{
${ }^{10}$ The newspapers are The Boston Globe, Chicago Tribune, The Daily Telegraph, Financial Times, The Globe and Mail, The Guardian, Los Angeles Times, The New York Times, The Times, The Wall Street Journal, and The Washington Post.
} 
Figure 1. Geopolitical risk (GPR) index over the study period

GPR

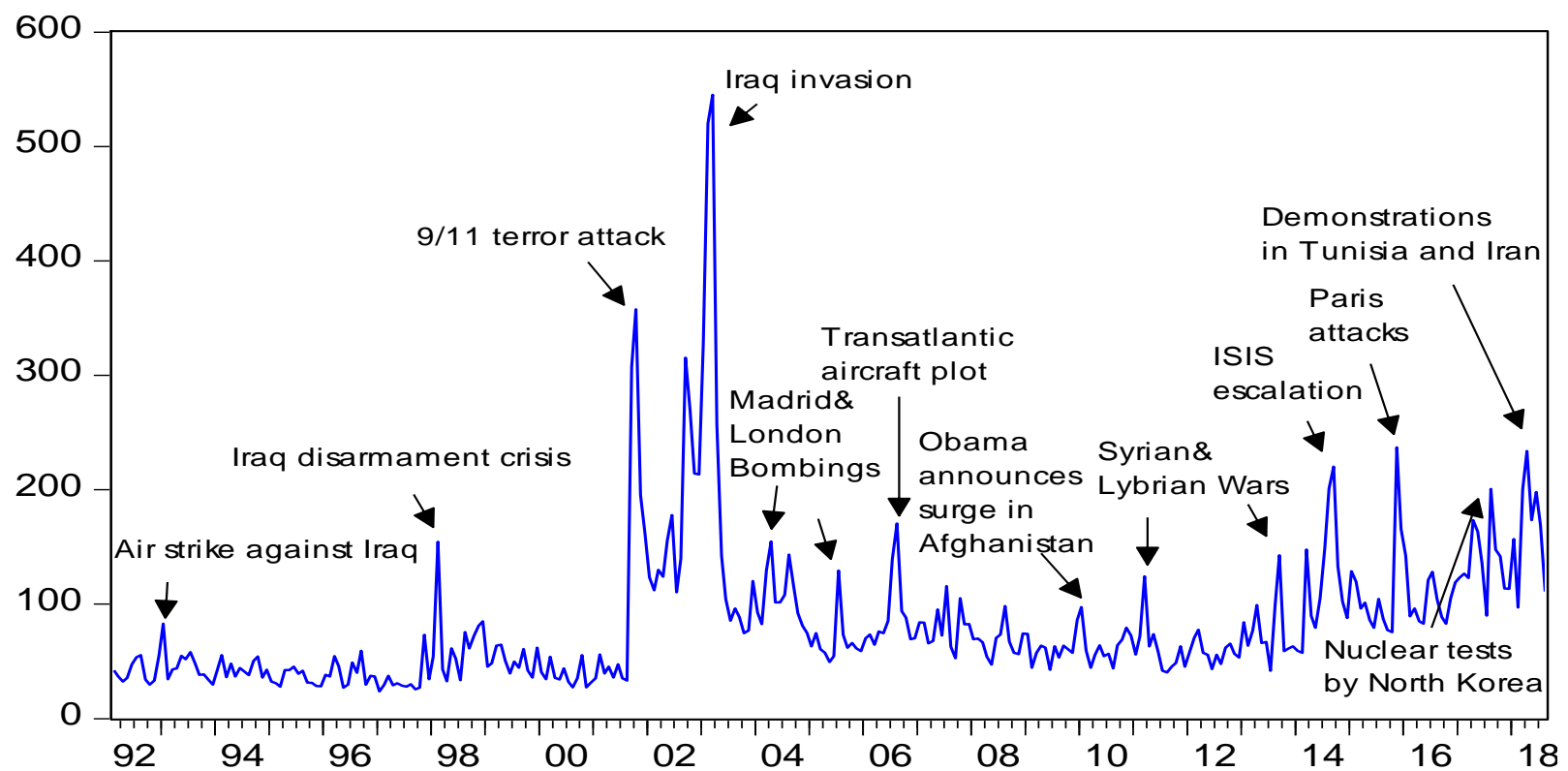

\section{Empirical Results}

\subsection{Global geopolitical risks and $T \& L$ stock index returns}

The empirical results are documented in Table 2. Panel A, B, C and D show the estimation results for Global, Asia\&Pacific, Europe and North America T\&L index returns, respectively. First, we estimate the parameters by applying conventional CLRM to assess the overall impact of geopolitical risks on T\&L stock returns. ${ }^{11}$ As stated earlier, the standard errors are computed based on heteroskedasticity consistent (HAC) estimation due to the presence of non-constant error variance in the data, which is a typical property of financial returns. The ordinary least squares (OLS) results are given in the first row of each panel. The parameters measuring the effects of GPR are all statistically significant at the $1 \%$ significance level, except for Asia\&Pacific region. The findings reveal a negative effect of changes in geopolitical risks on the index returns after controlling for the changes in VIX, oil prices and the crisis effect. More specifically, the OLS models suggest that a $1 \%$ increase in monthly geopolitical risk changes results in an approximate $0.02 \%$ decrease in Global, Europe and North America T\&L index returns.

As previously noted, the conventional OLS model enables us to find the average relationship between explained variables and a set of explanatory variables based on the conditional mean function, providing only a partial view of the response. Compared to a standard OLS model, QR technique provides a richer characterization of the data by measuring the response across different quantiles of the conditional distribution of the output variable. Looking at Panel A for

\footnotetext{
${ }^{11}$ We check the diagnostics of the OLS models by serial correlation test of Durbin-Watson and heteroscedasticity test of White. Based on Durbin-Watson tests, we fail to reject the null hypothesis of no autocorrelation. White test results show that we cannot reject the null hypothesis of homoscedasticity. Therefore, our OLS regressions seem to be well specified.
} 
the Global T\&L index, our results show that the quantile parameters measuring the sensitivity of the industry returns to geopolitical risks are negative and statistically significant in the lower quantiles $\left(\tau_{0.05}, \tau_{0.15}, \tau_{0.2}, \tau_{0.25}, \tau_{0.3}, \tau_{0.35}\right)$. Regarding the impact of geopolitical risks on the Asia\&Pacific T\&L index presented in Panel B, our findings do not document any significant effects. The results shown in Panel $\mathrm{C}$ suggest a negative and significant effect of geopolitical risks on European T\&L index in the lower quantiles $\left(\tau_{0.05}, \tau_{0.10}, \tau_{0.15}\right)$. As evidenced in Panel D, we find significantly negative coefficients for North America $T \& L$ index across lower parts of the conditional distribution $\left(\tau_{0.05}, \tau_{0.2}, \tau_{0.25}, \tau_{0.3}, \tau_{0.35}\right)$.

Figure 2 displays the quantile processes for each region. The horizontal axis exhibits the quantiles and the vertical axis shows the magnitude of the response. The red lines represent the $90 \%$ confidence intervals and the blue line draws the size of the reaction. It is worth noting that where the lower and upper bounds of the confidence intervals are in the same domain (below or above the zero line) - the estimated quantile coefficient is statistically significant. For example, the reaction of the Europe T\&L stock returns to changes in geopolitical risks is negative and statistically significant between 5 th and 15 th quantiles, but not significant between 20th and 95th quantiles. The plots also provide a visual representation of an asymmetric response. They demonstrate that the effect of geopolitical risks is higher in magnitude at the left tail of the return distribution where the $T \& L$ returns are negative.

Table 2 also provides evidence of significant effects of the control variables on the T\&L indices. A rise in VIX is found to be strongly and negatively affecting stock returns of T\&L companies, suggesting it is an important determinant of tourism sector returns across all regions. VIX is an implied volatility index which reflects expected future market volatility over the next month and widely used as an important proxy for investors' fear. As suggested by Dragouni et al. (2016), an increase in VIX adversely affects consumers' mood and their spending behavior; particularly, toward luxury goods, such as tourism. This naturally translates into a decline in returns of listed T\&L companies. The associated quantile coefficients are all statistically significant at the conventional levels and high in magnitude across all regions.

The CLRM results report significant and negative impacts of oil price changes, only on the Europe T\&L index. However, when we analyze the impact over the whole spectrum of the distribution, we find significant impacts in a few intermediate quantiles in case of Global and North America T\&L indices and in low, medium and high quantiles in Europe T\&L index. Oil price changes do not have any significant impact on Asia \& Pacific T\&L stock returns. The results reveal that European T\&L companies are particularly sensitive to oil price fluctuations regardless of the market state while the companies in North America and the Global index have an exposure under normal market conditions. This result suggests that European T\&L companies should use hedging strategies against undesirable oil price movements.

The linear model demonstrates that the crisis dummy variable, measuring the influence of 20072009 global financial crisis, is statistically significant and negative for the T\&L indices, except for the Asia\&Pacific index. The QR model shows significant effects across different quantiles. The crisis dummy variable is negative and statistically significant across almost all quantiles, except for the highest quantiles, in global and Europe T\&L indices. The impact is mostly concentrated in lower and intermediate quantiles for North American T\&L index and only in higher quantiles for Asia\&Pacific T\&L index. This suggests that tourism sector companies experienced a decline in their stock returns during the crisis, which is in line with large negative 
growth for service industries in the crisis period (Ender and Demir, 2018; Rao and Reddy, 2015).

We can assess whether the CLRM gives an adequate picture of the central tendencies featured in the data by comparing it with the median estimator $\left(\tau_{0.5}\right)$ (Marrocu et al., 2015). The median estimator is more robust to the existence of outliers and heteroskedasticity potentially present in the data. As can be seen, none of the median parameters, measuring the exposure of T\&L stocks to geopolitical risks, is statistically significant. This shows that geopolitical events do not drive T\&L stocks under normal market circumstances. However, the CLRM models suggest significant effects of geopolitical risks on T\&L indices, except for Asia\&Pacific region, at the central location of the data. Therefore, analyzing the sensitivity of T\&L industry to geopolitical events by using the linear mean models can be misleading as the comparison gives different conclusions.

Examining the empirical findings across the regional tourism indices, the results suggest that geopolitical risks do not affect T\&L stock returns in a uniform way as the response is found to be heterogeneous across the regions. We find that geopolitical risks have no impact on the distribution of Asia\&Pacific T\&L stock returns, which makes the region most resilient to geopolitical events. For the other T\&L indices, the significant effects are found to be concentrated in lower quantiles. The highest impact in magnitude is reported for Europe T\&L index (0.056) when the market is in bearish state $\left(\tau_{0.1}\right)$. The magnitudes of quantile coefficients at lower quantiles are much higher than those at higher quantiles, suggesting an asymmetric effect of geopolitical risks on the T\&L index returns. Therefore, the exposure of tourism sector to geopolitical risks intensifies during bear market conditions in which T\&L stock prices fall. ${ }^{12}$ The results and potential implications are further discussed in Section 5.

\footnotetext{
${ }^{12} \mathrm{We}$ also form joint hypotheses using parameters for more than one quantile. Accordingly, we use the slope equality tests by Koenker and Bassett (1982) across different quantiles. More specifically, we run pair-wise tests for each regressor and form a Wald test with a null hypothesis of equal coefficients; hence, we test whether a coefficient at a specific quantile is statistically different from another coefficient at any other quantile. Therefore, the higher the number of rejections of the null hypothesis of coefficient equality, the stronger the evidence supporting the hypothesis that the impact of geopolitical risks on T\&L stocks differ with respect to the quantile levels. The untabulated test findings suggest that the test results verify the findings from the QR regressions. We do not present the test results in the paper but they are available upon request.
} 
Table 2. CLRM and QR models results (Geopolitical Risks)

Panel A. Global Travel \& Leisure Index

\begin{tabular}{|c|c|c|c|c|c|c|c|c|c|c|}
\hline & $\mathrm{c}$ & t-ratio & GPR & t-ratio & VIX & t-ratio & OIL & t-ratio & CRISIS & t-ratio \\
\hline OLS & $0.007^{\mathrm{a}}$ & 3.294 & $-0.018^{\mathrm{a}}$ & -3.037 & $-0.117^{\mathrm{a}}$ & -10.080 & -0.016 & -0.599 & $-0.033^{a}$ & -3.782 \\
\hline 0.05 & $-0.057^{\mathrm{a}}$ & -9.365 & $-0.046^{\mathrm{a}}$ & -3.628 & $-0.146^{\mathrm{a}}$ & -3.964 & -0.022 & -0.345 & $-0.063^{b}$ & -1.966 \\
\hline 0.1 & $-0.038^{a}$ & -6.969 & -0.022 & -1.003 & $-0.137^{\mathrm{a}}$ & -4.241 & 0.029 & 0.506 & $-0.054^{\mathrm{a}}$ & -2.626 \\
\hline 0.15 & $-0.025^{\mathrm{a}}$ & -5.900 & $-0.030^{\mathrm{b}}$ & -2.393 & $-0.136^{\mathrm{a}}$ & -6.098 & 0.006 & 0.095 & $-0.067^{\mathrm{b}}$ & -2.581 \\
\hline 0.2 & $-0.017^{\mathrm{a}}$ & -5.360 & $-0.019^{b}$ & -2.441 & $-0.137^{a}$ & -7.009 & -0.031 & -0.797 & $-0.030^{\mathrm{a}}$ & -2.599 \\
\hline 0.25 & $-0.013^{a}$ & -4.380 & $-0.020^{\mathrm{a}}$ & -2.624 & $-0.127^{a}$ & -7.148 & -0.021 & -0.555 & $-0.028^{a}$ & -2.987 \\
\hline 0.3 & $-0.008^{a}$ & -3.020 & $-0.020^{b}$ & -2.577 & $-0.116^{\mathrm{a}}$ & -7.088 & -0.039 & -1.093 & $-0.034^{\mathrm{a}}$ & -3.184 \\
\hline 0.35 & -0.003 & -1.086 & $-0.013^{c}$ & -1.695 & $-0.116^{\mathrm{a}}$ & -7.556 & $-0.069^{b}$ & -2.117 & $-0.036^{\mathrm{a}}$ & -2.950 \\
\hline 0.4 & 0.002 & 0.685 & -0.012 & -1.576 & $-0.118^{a}$ & -8.231 & $-0.066^{\mathrm{b}}$ & -2.059 & $-0.033^{\mathrm{a}}$ & -3.051 \\
\hline 0.45 & $0.005^{\mathrm{c}}$ & 1.800 & -0.010 & -1.216 & $-0.122^{\mathrm{a}}$ & -8.346 & -0.051 & -1.556 & $-0.034^{\mathrm{a}}$ & -3.154 \\
\hline 0.5 & $0.008^{a}$ & 3.059 & -0.010 & -1.200 & $-0.114^{\mathrm{a}}$ & -8.033 & -0.043 & -1.323 & $-0.034^{\mathrm{a}}$ & -3.037 \\
\hline 0.55 & $0.014^{\mathrm{a}}$ & 5.728 & -0.010 & -1.244 & $-0.106^{\mathrm{a}}$ & -8.376 & -0.048 & -1.547 & $-0.030^{\mathrm{a}}$ & -2.613 \\
\hline 0.6 & $0.017^{\mathrm{a}}$ & 7.235 & -0.013 & -1.496 & $-0.108^{a}$ & -8.382 & $-0.059^{b}$ & -1.880 & $-0.031^{\mathrm{a}}$ & -2.809 \\
\hline 0.65 & $0.022^{\mathrm{a}}$ & 9.045 & -0.010 & -1.176 & $-0.102^{\mathrm{a}}$ & -7.641 & -0.031 & -0.996 & $-0.031^{\mathrm{a}}$ & -2.893 \\
\hline 0.7 & $0.026^{\mathrm{a}}$ & 10.492 & -0.010 & -1.046 & $-0.106^{\mathrm{a}}$ & -8.061 & -0.029 & -0.878 & $-0.032^{\mathrm{a}}$ & -3.121 \\
\hline 0.75 & $0.029^{\mathrm{a}}$ & 11.773 & -0.008 & -0.691 & $-0.108^{a}$ & -8.379 & -0.021 & -0.559 & $-0.032^{\mathrm{a}}$ & -3.200 \\
\hline 0.8 & $0.034^{\mathrm{a}}$ & 13.027 & -0.010 & -0.806 & $-0.106^{\mathrm{a}}$ & -8.786 & -0.004 & -0.122 & $-0.034^{\mathrm{a}}$ & -3.523 \\
\hline 0.85 & $0.041^{\mathrm{a}}$ & 13.075 & -0.001 & -0.091 & $-0.095^{\mathrm{a}}$ & -6.443 & 0.002 & 0.049 & -0.027 & -1.569 \\
\hline 0.9 & $0.051^{\mathrm{a}}$ & 14.222 & 0.006 & 0.584 & $-0.083^{\mathrm{a}}$ & -5.046 & -0.022 & -0.740 & $-0.027^{\mathrm{b}}$ & -2.204 \\
\hline 0.95 & $0.061^{\mathrm{a}}$ & 17.523 & 0.002 & 0.144 & $-0.082^{\mathrm{a}}$ & -4.529 & 0.013 & 0.324 & -0.014 & -0.697 \\
\hline \multicolumn{11}{|c|}{ Panel B. Asia-Pacific Travel \& Leisure Index } \\
\hline OLS & 0.002 & 0.831 & -0.008 & -1.060 & $-0.091^{\mathrm{a}}$ & -6.346 & 0.008 & 0.245 & -0.018 & -1.640 \\
\hline 0.05 & $-0.077^{\mathrm{a}}$ & -10.061 & -0.006 & -0.464 & $-0.094^{c}$ & -1.810 & -0.042 & -0.476 & 0.004 & 0.188 \\
\hline 0.1 & $-0.056^{\mathrm{a}}$ & -9.237 & -0.019 & -1.025 & $-0.084^{b}$ & -2.166 & -0.059 & -0.714 & -0.015 & -0.618 \\
\hline 0.15 & $-0.041^{a}$ & -8.385 & -0.021 & -1.124 & $-0.076^{a}$ & -2.725 & 0.006 & 0.087 & -0.014 & -0.713 \\
\hline 0.2 & $-0.029^{a}$ & -7.155 & -0.006 & -0.497 & $-0.073^{\mathrm{a}}$ & -3.529 & 0.040 & 0.747 & -0.014 & -1.105 \\
\hline
\end{tabular}




\begin{tabular}{|c|c|c|c|c|c|c|c|c|c|c|}
\hline 0.25 & $-0.022^{a}$ & -6.272 & -0.005 & -0.513 & $-0.085^{a}$ & -4.780 & 0.051 & 1.148 & -0.012 & -1.020 \\
\hline 0.3 & $-0.014^{a}$ & -4.620 & -0.004 & -0.567 & $-0.083^{a}$ & -6.562 & 0.039 & 1.066 & -0.015 & -1.365 \\
\hline 0.35 & $-0.009^{a}$ & -3.139 & -0.004 & -0.541 & $-0.091^{a}$ & -7.755 & 0.017 & 0.485 & -0.019 & -1.625 \\
\hline 0.4 & $-0.005^{\mathrm{c}}$ & -1.799 & -0.004 & -0.454 & $-0.092^{a}$ & -8.040 & 0.006 & 0.175 & -0.016 & -1.319 \\
\hline 0.45 & -0.001 & -0.354 & -0.002 & -0.287 & $-0.088^{a}$ & -7.534 & 0.030 & 0.807 & -0.010 & -0.992 \\
\hline 0.5 & 0.004 & 1.289 & 0.001 & 0.096 & $-0.093^{a}$ & -7.909 & 0.018 & 0.504 & -0.011 & -1.076 \\
\hline 0.55 & $0.008^{\mathrm{a}}$ & 2.948 & 0.006 & 0.744 & $-0.097^{\mathrm{a}}$ & -7.913 & -0.005 & -0.144 & -0.014 & -1.242 \\
\hline 0.6 & $0.011^{\mathrm{a}}$ & 3.921 & 0.007 & 0.755 & $-0.092^{a}$ & -6.865 & -0.011 & -0.313 & -0.016 & -1.489 \\
\hline 0.65 & $0.017^{\mathrm{a}}$ & 5.913 & 0.008 & 0.922 & $-0.093^{a}$ & -5.682 & 0.012 & 0.323 & -0.010 & -0.978 \\
\hline 0.7 & $0.023^{\mathrm{a}}$ & 7.598 & 0.009 & 0.921 & $-0.094^{a}$ & -4.891 & -0.004 & -0.099 & -0.013 & -1.425 \\
\hline 0.75 & $0.031^{\mathrm{a}}$ & 8.887 & 0.003 & 0.263 & $-0.101^{\mathrm{a}}$ & -3.900 & -0.030 & -0.734 & $-0.017^{\mathrm{c}}$ & -1.852 \\
\hline 0.8 & $0.037^{\mathrm{a}}$ & 9.889 & 0.013 & 1.036 & $-0.101^{a}$ & -3.744 & -0.010 & -0.236 & $-0.019^{b}$ & -2.065 \\
\hline 0.85 & $0.045^{\mathrm{a}}$ & 11.364 & 0.016 & 1.430 & $-0.077^{a}$ & -2.634 & -0.018 & -0.445 & $-0.023^{b}$ & -2.382 \\
\hline 0.9 & $0.056^{\mathrm{a}}$ & 12.208 & 0.016 & 1.448 & $-0.086^{a}$ & -2.966 & -0.013 & -0.323 & $-0.033^{\mathrm{a}}$ & -3.841 \\
\hline 0.95 & $0.078^{\mathrm{a}}$ & 11.402 & 0.000 & 0.000 & $-0.077^{\mathrm{b}}$ & -2.278 & 0.000 & -0.005 & $-0.046^{a}$ & -3.033 \\
\hline \multicolumn{11}{|c|}{ Panel C. Europe Travel \& Leisure Index } \\
\hline OLS & $0.007^{\mathrm{b}}$ & 2.579 & $-0.019^{b}$ & -2.568 & $-0.122^{\mathrm{a}}$ & -8.420 & $-0.079^{\mathrm{b}}$ & -2.405 & $-0.052^{\mathrm{a}}$ & -4.756 \\
\hline 0.05 & $-0.077^{\mathrm{a}}$ & -10.932 & $-0.042^{\mathrm{a}}$ & -5.082 & $-0.208^{a}$ & -8.399 & $-0.161^{b}$ & -2.198 & $-0.072^{\mathrm{b}}$ & -1.801 \\
\hline 0.1 & $-0.054^{a}$ & -9.290 & $-0.056^{\mathrm{a}}$ & -6.855 & $-0.163^{a}$ & -7.101 & $-0.087^{\mathrm{c}}$ & -1.773 & $-0.053^{\mathrm{a}}$ & -3.901 \\
\hline 0.15 & $-0.034^{a}$ & -5.829 & $-0.028^{c}$ & -1.728 & $-0.150^{a}$ & -5.149 & -0.052 & -1.036 & $-0.064^{\mathrm{a}}$ & -4.573 \\
\hline 0.2 & $-0.023^{a}$ & -5.722 & -0.020 & -1.455 & $-0.126^{\mathrm{a}}$ & -5.979 & -0.047 & -1.030 & $-0.068^{a}$ & -3.620 \\
\hline 0.25 & $-0.015^{a}$ & -4.407 & -0.015 & -1.237 & $-0.118^{a}$ & -7.040 & $-0.110^{\mathrm{b}}$ & -2.176 & $-0.067^{\mathrm{a}}$ & -3.018 \\
\hline 0.3 & $-0.011^{a}$ & -3.325 & -0.012 & -1.169 & $-0.127^{a}$ & -7.891 & $-0.111^{b}$ & -2.495 & $-0.053^{a}$ & -3.515 \\
\hline 0.35 & -0.004 & -1.440 & -0.006 & -0.695 & $-0.131^{a}$ & -8.560 & $-0.111^{\mathrm{a}}$ & -2.702 & $-0.055^{\mathrm{a}}$ & -3.854 \\
\hline 0.4 & -0.001 & -0.404 & -0.006 & -0.698 & $-0.126^{a}$ & -8.188 & $-0.118^{a}$ & -2.923 & $-0.056^{a}$ & -4.046 \\
\hline 0.45 & $0.005^{\mathrm{c}}$ & 1.872 & -0.010 & -1.134 & $-0.117^{a}$ & -7.567 & $-0.103^{b}$ & -2.576 & $-0.058^{a}$ & -4.263 \\
\hline 0.5 & $0.009^{\mathrm{a}}$ & 3.100 & -0.012 & -1.384 & $-0.114^{a}$ & -7.427 & $-0.095^{\mathrm{b}}$ & -2.450 & $-0.055^{\mathrm{a}}$ & -4.488 \\
\hline 0.55 & $0.014^{\mathrm{a}}$ & 4.803 & -0.010 & -1.145 & $-0.104^{a}$ & -6.965 & $-0.101^{\mathrm{a}}$ & -2.668 & $-0.055^{\mathrm{a}}$ & -4.247 \\
\hline 0.6 & $0.019^{\mathrm{a}}$ & 6.341 & -0.006 & -0.664 & $-0.099^{a}$ & -6.336 & $-0.093^{b}$ & -2.506 & $-0.053^{a}$ & -4.397 \\
\hline
\end{tabular}




\begin{tabular}{|c|c|c|c|c|c|c|c|c|c|c|}
\hline 0.65 & $0.024^{\mathrm{a}}$ & 7.799 & -0.005 & -0.559 & $-0.099^{a}$ & -5.950 & $-0.064^{\mathrm{c}}$ & -1.695 & $-0.049^{a}$ & -3.828 \\
\hline 0.7 & $0.029^{\mathrm{a}}$ & 9.509 & -0.008 & -0.937 & $-0.098^{\mathrm{a}}$ & -5.738 & $-0.063^{c}$ & -1.665 & $-0.053^{a}$ & -4.348 \\
\hline 0.75 & $0.036^{\mathrm{a}}$ & 11.193 & -0.002 & -0.255 & $-0.074^{\mathrm{a}}$ & -3.360 & -0.057 & -1.556 & $-0.056^{\mathrm{a}}$ & -4.605 \\
\hline 0.8 & $0.042^{\mathrm{a}}$ & 12.947 & 0.000 & -0.038 & $-0.066^{\mathrm{a}}$ & -2.964 & $-0.070^{b}$ & -1.988 & $-0.060^{a}$ & -5.001 \\
\hline 0.85 & $0.049^{\mathrm{a}}$ & 15.305 & -0.007 & -0.511 & $-0.078^{a}$ & -3.078 & -0.060 & -1.455 & $-0.052^{a}$ & -2.748 \\
\hline 0.9 & $0.059^{\mathrm{a}}$ & 15.422 & -0.003 & -0.204 & $-0.073^{\mathrm{a}}$ & -2.750 & -0.014 & -0.318 & -0.038 & -1.456 \\
\hline 0.95 & $0.071^{\mathrm{a}}$ & 15.420 & 0.017 & 1.179 & $-0.059^{\mathrm{b}}$ & -2.229 & -0.026 & -0.630 & -0.028 & -1.217 \\
\hline \multicolumn{11}{|c|}{ Panel D. North America Travel \& Leisure Index } \\
\hline OLS & $0.010^{\mathrm{a}}$ & 3.847 & $-0.020^{\mathrm{a}}$ & -2.864 & $-0.141^{\mathrm{a}}$ & -10.777 & -0.037 & -1.229 & $-0.033^{\mathrm{a}}$ & -3.294 \\
\hline 0.05 & $-0.065^{\mathrm{a}}$ & -10.307 & $-0.043^{a}$ & -5.352 & $-0.152^{\mathrm{a}}$ & -5.383 & 0.004 & 0.063 & $-0.050^{\mathrm{b}}$ & -2.551 \\
\hline 0.1 & $-0.041^{\mathrm{a}}$ & -7.862 & -0.021 & -1.402 & $-0.151^{\mathrm{a}}$ & -5.405 & -0.032 & -0.457 & $-0.065^{\mathrm{a}}$ & -3.458 \\
\hline 0.15 & $-0.029^{a}$ & -7.829 & -0.017 & -1.540 & $-0.149^{a}$ & -6.277 & -0.059 & -1.263 & $-0.077^{\mathrm{a}}$ & -4.022 \\
\hline 0.2 & $-0.023^{a}$ & -6.337 & $-0.021^{b}$ & -2.013 & $-0.162^{\mathrm{a}}$ & -6.904 & -0.054 & -1.203 & $-0.082^{a}$ & -3.553 \\
\hline 0.25 & $-0.017^{\mathrm{a}}$ & -4.670 & $-0.025^{\mathrm{b}}$ & -2.329 & $-0.166^{\mathrm{a}}$ & -6.541 & -0.074 & -1.580 & -0.062 & -1.071 \\
\hline 0.3 & $-0.010^{\mathrm{a}}$ & -2.965 & $-0.022^{b}$ & -2.087 & $-0.147^{\mathrm{a}}$ & -6.337 & -0.072 & -1.621 & -0.044 & -1.262 \\
\hline 0.35 & 0.000 & -0.102 & $-0.018^{c}$ & -1.667 & $-0.124^{\mathrm{a}}$ & -5.850 & -0.065 & -1.455 & $-0.043^{\mathrm{b}}$ & -1.997 \\
\hline 0.4 & 0.004 & 1.408 & -0.014 & -1.374 & $-0.124^{\mathrm{a}}$ & -6.215 & $-0.091^{\mathrm{b}}$ & -2.132 & -0.031 & -1.478 \\
\hline 0.45 & $0.008^{\mathrm{a}}$ & 2.653 & -0.012 & -1.184 & $-0.128^{a}$ & -6.361 & -0.069 & -1.597 & $-0.035^{c}$ & -1.750 \\
\hline 0.5 & $0.011^{\mathrm{a}}$ & 3.968 & -0.007 & -0.689 & $-0.133^{\mathrm{a}}$ & -6.907 & $-0.085^{b}$ & -2.064 & $-0.033^{c}$ & -1.737 \\
\hline 0.55 & $0.015^{\mathrm{a}}$ & 5.207 & -0.005 & -0.531 & $-0.138^{\mathrm{a}}$ & -7.903 & $-0.077^{\mathrm{b}}$ & -2.026 & -0.019 & -1.091 \\
\hline 0.6 & $0.020^{\mathrm{a}}$ & 7.277 & -0.007 & -0.742 & $-0.128^{\mathrm{a}}$ & -8.625 & $-0.096^{\mathrm{a}}$ & -2.777 & -0.020 & -1.293 \\
\hline 0.65 & $0.026^{\mathrm{a}}$ & 9.011 & -0.012 & -1.177 & $-0.132^{\mathrm{a}}$ & -9.026 & $-0.074^{b}$ & -2.076 & -0.018 & -1.120 \\
\hline 0.7 & $0.030^{\mathrm{a}}$ & 10.247 & -0.009 & -0.797 & $-0.122^{\mathrm{a}}$ & -9.170 & -0.052 & -1.390 & -0.020 & -1.149 \\
\hline 0.75 & $0.036^{\mathrm{a}}$ & 11.476 & -0.016 & -1.451 & $-0.126^{\mathrm{a}}$ & -9.082 & -0.030 & -0.841 & -0.007 & -0.498 \\
\hline 0.8 & $0.043^{\mathrm{a}}$ & 13.490 & -0.013 & -1.161 & $-0.124^{\mathrm{a}}$ & -9.042 & -0.041 & -1.149 & -0.013 & -0.943 \\
\hline 0.85 & $0.050^{\mathrm{a}}$ & 14.788 & -0.005 & -0.450 & $-0.128^{a}$ & -9.452 & -0.051 & -1.453 & -0.015 & -1.283 \\
\hline 0.9 & $0.060^{\mathrm{a}}$ & 15.971 & -0.012 & -0.837 & $-0.128^{a}$ & -8.520 & 0.015 & 0.453 & -0.001 & -0.035 \\
\hline 0.95 & $0.073^{\mathrm{a}}$ & 17.149 & -0.019 & -1.459 & $-0.096^{\mathrm{a}}$ & -3.548 & 0.013 & 0.272 & -0.003 & -0.172 \\
\hline
\end{tabular}


Figure 2. Plots of quantile regression estimates (Geopolitical risks)

GLOBAL

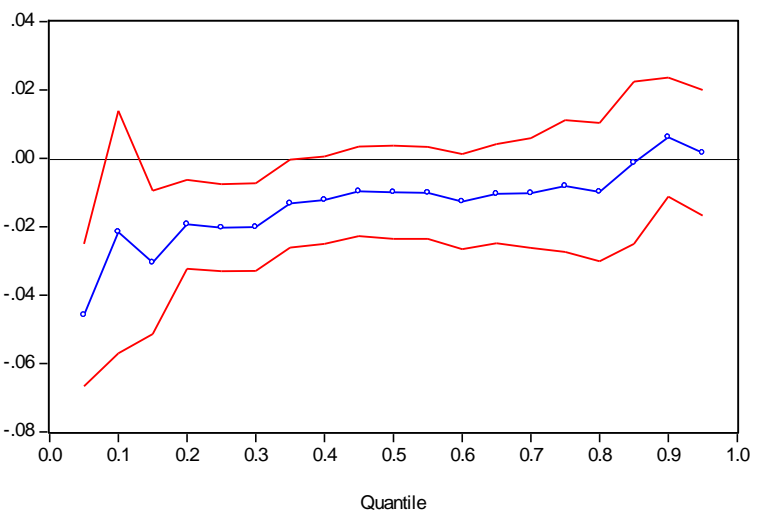

EUROPE

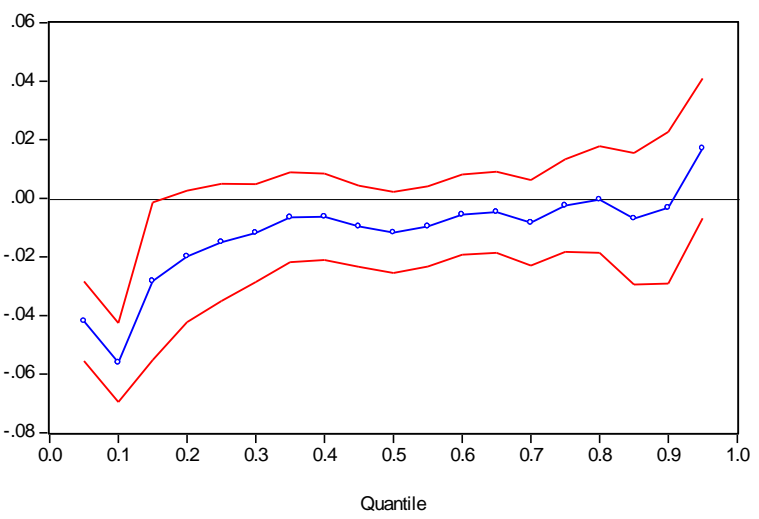

ASIA\&PACIFIC

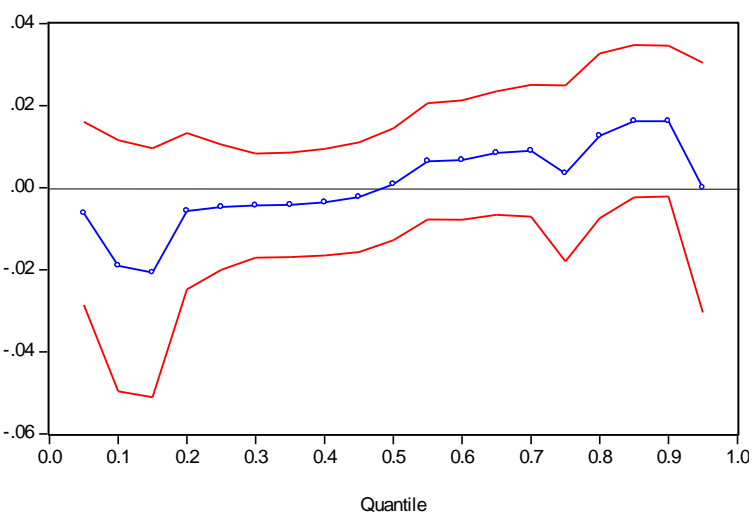

NORTH AMERICA

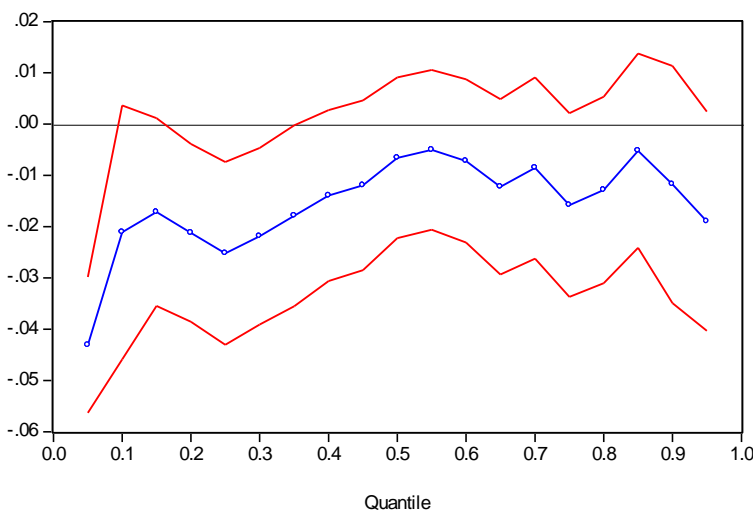

\subsection{Global geopolitical threats, acts and T\&L stock index returns}

In this section, we analyze whether the impacts of geopolitical risks are due to threats of adverse events or to their realizations. For this, two sub-indexes constructed by Caldara and Iacoviello (2018) are used, enabling us to distinguish between geopolitical threats (GPT) and acts (GPA). The GPT index represents the realization of adverse geopolitical events, while the GPA index captures geopolitical threats, such as tensions increasing after terror incidences or before wars.

Table 3 presents the estimation results for the model analyzing the effects of threats. The CLRM coefficients suggest that the Asia\&Pacific tourism index is resilient to GPR threats, while the other index returns are negatively affected. The linear regression parameters are statistically significant at the 5\% level for Europe and North America and at the 1\% level for the Global tourism index - the results indicate that a $1 \%$ increase in monthly geopolitical risk changes will result in an around $0.016 \%$ decrease in these indices' returns.

The estimation results in Table 3 and quantile processes in Figure 3 suggest no impact of the GPR threats on the distribution of the Asia\&Pacific tourism index returns. ${ }^{13}$ The effects of geopolitical threats vary across quantiles for the other T\&L indices. The negative and significant impact is evident for lower quantiles; $\tau_{0.05}, \tau_{0.15}, \tau_{0.2}, \tau_{0.25}, \tau_{0.3}$ for the Global T\&L index, $\tau_{0.05}, \tau_{0.1}, \tau_{0.15}$ for the European $T \& L$ index and $\tau_{0.05}, \tau_{0.2}, \tau_{0.25}, \tau_{0.3}$ for the North America T\&L index. The quantile coefficients are statistically significant and higher in magnitude for

\footnotetext{
${ }^{13}$ The results regarding the impacts of control variables on T\&L stock returns are similar under different models.
} 
lower quantiles, showing that the impacts of geopolitical threats intensify when the T\&L stocks are under bearish market state. Therefore, the empirical results imply the presence of a statedependent effect of geopolitical threats on T\&L stock performance.

Table 4 documents the estimation results for the model measuring the impacts of geopolitical acts. The CLRM parameters are all statically significant at the $1 \%$ level, with the exception of the Asia\&Pacific index. The coefficients are all negative showing that actual geopolitical events lead to a reduction in tourism stock returns. Regarding the impacts of geopolitical acts across different quantiles depicted in Table 4 and Figure 4, we find insignificant effects for the Asia\&Pacific index. For the other indices, the findings demonstrate significant and negative impacts conditional on the location of the return distribution. The quantile parameters are statistically significant and negative over the quantile range of $\tau_{0.05^{-}} \tau_{0.65}$, except for $\tau_{0.1}$, for the Global T\&L index. The Europe and North American T\&L index returns exhibit significant drops at $\tau_{0.1}, \tau_{0.2}, \tau_{0.5}, \tau_{0.55}, \tau_{0.6}, \tau_{0.65}, \tau_{0.7}, \tau_{0.75}$ and $\tau_{0.05}, \tau_{0.1}, \tau_{1.5}, \tau_{0.25}, \tau_{0.6}, \tau_{0.65}, \tau_{0.7}, \tau_{0.75}$, respectively. The results reveal that the negative impacts of geopolitical acts remain significant regardless of bearish, bullish or normal market circumstances. In other words, the materialization of the geopolitical risks adversely influences T\&L industry in all market states.

When we compare the results in terms of magnitude of the quantile coefficients, we observe a greater sensitivity of T\&L stock returns to geopolitical threats compared to actual events only at lower quantiles. This finding suggests that response of T\&L stocks to geopolitical threats is strong only during periods of falling T\&L stock prices. The realization of adverse geopolitical events is found to produce relatively smaller effects on the index returns at lower quantiles; however, the effect is still strong at intermediate and higher quantiles. This shows that the adverse effects of geopolitical risk on T\&L stocks are driven more by the threat of geopolitical events when the T\&L stocks are falling. The realization of geopolitical events significantly affects T\&L stocks under all market states, even though it produces relatively small effects during bearish times. 
Table 3. CLRM and QR models results (Geopolitical Threats)

\begin{tabular}{|c|c|c|c|c|c|c|c|c|c|c|}
\hline & $\mathrm{c}$ & t-ratio & GPR-Threats & t-ratio & VIX & t-ratio & OIL & t-ratio & CRISIS & t-ratio \\
\hline OLS & $0.007^{\mathrm{a}}$ & 3.272 & $-0.015^{\mathrm{a}}$ & -2.637 & $-0.116^{\mathrm{a}}$ & -10.033 & -0.017 & -0.634 & $-0.033^{\mathrm{a}}$ & -3.754 \\
\hline 0.05 & $-0.059^{a}$ & -8.573 & $-0.038^{b}$ & -2.145 & $-0.160^{\mathrm{a}}$ & -5.036 & -0.007 & -0.124 & $-0.050^{b}$ & -2.289 \\
\hline 0.1 & $-0.038^{a}$ & -6.712 & -0.017 & -0.842 & $-0.141^{\mathrm{a}}$ & -3.928 & 0.017 & 0.279 & $-0.057^{a}$ & -2.777 \\
\hline 0.15 & $-0.023^{\mathrm{a}}$ & -6.787 & $-0.021^{b}$ & -2.341 & $-0.150^{\mathrm{a}}$ & -6.698 & -0.013 & -0.282 & $-0.072^{\mathrm{a}}$ & -3.490 \\
\hline 0.2 & $-0.018^{a}$ & -5.629 & $-0.017^{b}$ & -2.236 & $-0.138^{a}$ & -7.216 & -0.012 & -0.316 & $-0.030^{a}$ & -2.739 \\
\hline 0.25 & $-0.013^{\mathrm{a}}$ & -4.228 & $-0.016^{\mathrm{b}}$ & -2.199 & $-0.120^{\mathrm{a}}$ & -6.648 & -0.037 & -0.965 & $-0.034^{\mathrm{a}}$ & -2.968 \\
\hline 0.3 & $-0.008^{a}$ & -3.005 & $-0.016^{b}$ & -2.081 & $-0.113^{a}$ & -6.674 & -0.037 & -1.027 & $-0.036^{a}$ & -3.212 \\
\hline 0.35 & -0.003 & -1.102 & -0.012 & -1.621 & $-0.115^{\mathrm{a}}$ & -7.372 & $-0.062^{c}$ & -1.897 & $-0.036^{a}$ & -2.972 \\
\hline 0.4 & 0.001 & 0.463 & -0.009 & -1.337 & $-0.122^{a}$ & -8.228 & $-0.064^{c}$ & -1.910 & $-0.032^{\mathrm{a}}$ & -2.905 \\
\hline 0.45 & $0.005^{c}$ & 1.833 & -0.008 & -1.033 & $-0.120^{\mathrm{a}}$ & -8.171 & -0.050 & -1.489 & $-0.033^{a}$ & -3.036 \\
\hline 0.5 & $0.008^{\mathrm{a}}$ & 3.300 & -0.008 & -1.091 & $-0.114^{\mathrm{a}}$ & -8.180 & -0.048 & -1.471 & $-0.034^{\mathrm{a}}$ & -2.998 \\
\hline 0.55 & $0.014^{\mathrm{a}}$ & 5.756 & -0.009 & -1.207 & $-0.107^{\mathrm{a}}$ & -8.433 & -0.046 & -1.478 & $-0.030^{\mathrm{a}}$ & -2.666 \\
\hline 0.6 & $0.018^{\mathrm{a}}$ & 7.357 & -0.006 & -0.738 & $-0.101^{a}$ & -7.570 & $-0.054^{c}$ & -1.694 & $-0.031^{b}$ & -2.560 \\
\hline 0.65 & $0.022^{\mathrm{a}}$ & 8.976 & -0.007 & -0.876 & $-0.103^{a}$ & -7.591 & -0.040 & -1.253 & $-0.026^{b}$ & -2.373 \\
\hline 0.7 & $0.026^{\mathrm{a}}$ & 10.574 & -0.005 & -0.476 & $-0.108^{\mathrm{a}}$ & -7.731 & -0.042 & -1.149 & $-0.031^{\mathrm{a}}$ & -2.884 \\
\hline 0.75 & $0.030^{\mathrm{a}}$ & 11.782 & -0.006 & -0.509 & $-0.108^{\mathrm{a}}$ & -8.263 & -0.024 & -0.671 & $-0.033^{\mathrm{a}}$ & -3.222 \\
\hline 0.8 & $0.033^{\mathrm{a}}$ & 12.843 & -0.004 & -0.383 & -0.109 & -8.853 & -0.004 & -0.117 & $-0.034^{\mathrm{a}}$ & -3.296 \\
\hline 0.85 & $0.041^{\mathrm{a}}$ & 13.091 & 0.001 & 0.100 & $-0.093^{\mathrm{a}}$ & -5.508 & -0.013 & -0.345 & -0.026 & -1.523 \\
\hline 0.9 & $0.051^{\mathrm{a}}$ & 14.151 & 0.007 & 0.726 & $-0.086^{\mathrm{a}}$ & -5.209 & -0.026 & -0.914 & $-0.027^{b}$ & -2.295 \\
\hline 0.95 & $0.061^{\mathrm{a}}$ & 17.557 & 0.002 & 0.161 & $-0.082^{a}$ & -4.552 & 0.013 & 0.325 & -0.015 & -0.668 \\
\hline \multicolumn{11}{|c|}{ Panel B. Asia-Pacific Travel \& Leisure Index } \\
\hline OLS & 0.002 & 0.828 & -0.006 & -0.878 & $-0.091^{\mathrm{a}}$ & -6.339 & 0.007 & 0.231 & -0.018 & -1.633 \\
\hline 0.05 & $-0.077^{a}$ & -9.860 & -0.006 & -0.499 & $-0.097^{c}$ & -1.811 & -0.034 & -0.378 & 0.003 & 0.141 \\
\hline 0.1 & $-0.056^{a}$ & -8.915 & -0.011 & -0.580 & -0.072 & -1.634 & 0.000 & -0.001 & -0.001 & -0.051 \\
\hline 0.15 & $-0.039^{a}$ & -7.875 & -0.008 & -0.403 & $-0.078^{a}$ & -2.754 & 0.006 & 0.075 & -0.014 & -0.664 \\
\hline 0.2 & $-0.029^{a}$ & -7.163 & -0.005 & -0.502 & $-0.072^{a}$ & -3.622 & 0.038 & 0.727 & -0.013 & -1.084 \\
\hline
\end{tabular}




\begin{tabular}{|c|c|c|c|c|c|c|c|c|c|c|}
\hline 0.25 & $-0.021^{a}$ & -6.205 & -0.003 & -0.409 & $-0.087^{\mathrm{a}}$ & -5.030 & 0.047 & 1.081 & -0.014 & -1.103 \\
\hline 0.3 & $-0.014^{\mathrm{a}}$ & -4.667 & -0.004 & -0.529 & $-0.082^{a}$ & -6.382 & 0.042 & 1.122 & -0.015 & -1.332 \\
\hline 0.35 & $-0.008^{a}$ & -2.888 & -0.002 & -0.288 & $-0.096^{a}$ & -8.472 & 0.007 & 0.183 & -0.013 & -1.163 \\
\hline 0.4 & $-0.005^{b}$ & -1.778 & -0.003 & -0.448 & $-0.092^{a}$ & -8.099 & 0.006 & 0.160 & -0.009 & -0.830 \\
\hline 0.45 & -0.001 & -0.228 & 0.000 & -0.024 & $-0.089^{a}$ & -7.601 & 0.024 & 0.672 & -0.012 & -1.112 \\
\hline 0.5 & 0.004 & 1.381 & 0.003 & 0.387 & $-0.092^{a}$ & -7.691 & 0.012 & 0.322 & -0.012 & -1.110 \\
\hline 0.55 & $0.008^{\mathrm{a}}$ & 2.913 & 0.006 & 0.762 & $-0.097^{\mathrm{a}}$ & -7.920 & -0.008 & -0.235 & -0.013 & -1.240 \\
\hline 0.6 & $0.011^{\mathrm{a}}$ & 3.939 & 0.005 & 0.624 & $-0.096^{a}$ & -7.438 & -0.015 & -0.448 & -0.016 & -1.514 \\
\hline 0.65 & $0.017^{\mathrm{a}}$ & 5.887 & 0.007 & 0.861 & $-0.093^{a}$ & -5.602 & 0.009 & 0.247 & -0.010 & -0.946 \\
\hline 0.7 & $0.023^{\mathrm{a}}$ & 7.586 & 0.008 & 0.875 & $-0.095^{\mathrm{a}}$ & -5.067 & -0.003 & -0.082 & -0.015 & -1.565 \\
\hline 0.75 & $0.031^{\mathrm{a}}$ & 8.891 & 0.003 & 0.248 & $-0.101^{a}$ & -3.959 & -0.028 & -0.693 & $-0.017^{\mathrm{c}}$ & -1.814 \\
\hline 0.8 & $0.037^{\mathrm{a}}$ & 9.808 & 0.013 & 1.139 & $-0.099^{\mathrm{a}}$ & -3.772 & -0.006 & -0.151 & $-0.019^{\mathrm{b}}$ & -2.006 \\
\hline 0.85 & $0.044^{\mathrm{a}}$ & 11.472 & 0.008 & 0.711 & $-0.080^{a}$ & -2.949 & -0.031 & -0.871 & $-0.026^{a}$ & -3.235 \\
\hline 0.9 & $0.054^{\mathrm{a}}$ & 12.485 & 0.015 & 1.373 & $-0.080^{a}$ & -2.776 & -0.019 & -0.515 & $-0.031^{\mathrm{a}}$ & -3.599 \\
\hline 0.95 & $0.079^{\mathrm{a}}$ & 11.402 & 0.014 & 0.778 & $-0.062^{b}$ & -2.022 & -0.039 & -1.196 & $-0.055^{\mathrm{a}}$ & -6.115 \\
\hline \multicolumn{11}{|c|}{ Panel C. Europe Travel \& Leisure Index } \\
\hline OLS & $0.007^{\mathrm{a}}$ & 2.565 & $-0.016^{\mathrm{b}}$ & -2.179 & $-0.122^{\mathrm{a}}$ & -8.386 & $-0.080^{\mathrm{b}}$ & -2.430 & $-0.052^{\mathrm{a}}$ & -4.729 \\
\hline 0.05 & $-0.082^{a}$ & -10.844 & $-0.037^{\mathrm{a}}$ & -4.695 & $-0.214^{\mathrm{a}}$ & -8.509 & $-0.142^{c}$ & -1.792 & -0.061 & -1.428 \\
\hline 0.1 & $-0.051^{a}$ & -7.395 & $-0.048^{\mathrm{a}}$ & -5.005 & $-0.159^{a}$ & -5.473 & $-0.099^{b}$ & -2.006 & $-0.056^{\mathrm{a}}$ & -4.103 \\
\hline 0.15 & $-0.034^{\mathrm{a}}$ & -6.184 & $-0.026^{c}$ & -1.863 & $-0.149^{a}$ & -5.492 & -0.055 & -1.163 & $-0.064^{\mathrm{a}}$ & -4.521 \\
\hline 0.2 & $-0.024^{\mathrm{a}}$ & -5.871 & -0.020 & -1.610 & $-0.127^{a}$ & -6.213 & -0.051 & -1.122 & $-0.067^{\mathrm{a}}$ & -3.516 \\
\hline 0.25 & $-0.016^{\mathrm{a}}$ & -4.456 & -0.013 & -1.183 & $-0.119^{a}$ & -6.966 & $-0.108^{b}$ & -2.080 & $-0.067^{a}$ & -2.799 \\
\hline 0.3 & $-0.010^{a}$ & -3.086 & -0.010 & -1.074 & $-0.128^{a}$ & -8.068 & $-0.114^{\mathrm{a}}$ & -2.661 & $-0.053^{a}$ & -3.685 \\
\hline 0.35 & -0.004 & -1.364 & -0.004 & -0.511 & $-0.130^{a}$ & -8.517 & $-0.114^{a}$ & -2.808 & $-0.055^{\mathrm{a}}$ & -3.962 \\
\hline 0.4 & -0.001 & -0.275 & -0.005 & -0.640 & $-0.124^{a}$ & -8.099 & $-0.123^{a}$ & -3.122 & $-0.055^{a}$ & -4.196 \\
\hline 0.45 & $0.006^{b}$ & 2.119 & -0.007 & -0.903 & $-0.115^{a}$ & -7.564 & $-0.103^{a}$ & -2.631 & $-0.059^{a}$ & -4.364 \\
\hline 0.5 & $0.009^{\mathrm{a}}$ & 3.116 & -0.004 & -0.538 & $-0.115^{a}$ & -7.396 & $-0.103^{a}$ & -2.704 & $-0.054^{\mathrm{a}}$ & -4.371 \\
\hline 0.55 & $0.015^{\mathrm{a}}$ & 5.109 & -0.003 & -0.445 & $-0.102^{a}$ & -6.663 & $-0.114^{a}$ & -3.005 & $-0.056^{a}$ & -4.268 \\
\hline 0.6 & $0.019^{\mathrm{a}}$ & 6.287 & -0.003 & -0.435 & $-0.102^{a}$ & -6.503 & $-0.093^{b}$ & -2.495 & $-0.053^{a}$ & -4.366 \\
\hline
\end{tabular}




\begin{tabular}{|c|c|c|c|c|c|c|c|c|c|c|}
\hline 0.65 & $0.024^{\mathrm{a}}$ & 7.652 & -0.002 & -0.272 & $-0.097^{\mathrm{a}}$ & -5.895 & $-0.063^{c}$ & -1.657 & $-0.048^{a}$ & -3.735 \\
\hline 0.7 & $0.029^{a}$ & 9.537 & -0.004 & -0.490 & $-0.101^{a}$ & -5.932 & -0.053 & -1.408 & $-0.051^{a}$ & -4.075 \\
\hline 0.75 & $0.037^{a}$ & 11.386 & 0.003 & 0.266 & $-0.066^{\mathrm{a}}$ & -3.010 & $-0.069^{c}$ & -1.856 & $-0.055^{\mathrm{a}}$ & -4.310 \\
\hline 0.8 & $0.042^{\mathrm{a}}$ & 13.048 & 0.000 & 0.010 & $-0.066^{\mathrm{a}}$ & -2.989 & $-0.067^{\mathrm{c}}$ & -1.874 & $-0.060^{a}$ & -5.137 \\
\hline 0.85 & $0.049^{\mathrm{a}}$ & 15.103 & -0.008 & -0.559 & $-0.083^{a}$ & -3.256 & -0.064 & -1.483 & $-0.053^{a}$ & -2.772 \\
\hline 0.9 & $0.059^{a}$ & 15.691 & -0.003 & -0.172 & $-0.074^{\mathrm{a}}$ & -2.844 & -0.016 & -0.366 & -0.038 & -1.453 \\
\hline 0.95 & $0.072^{\mathrm{a}}$ & 15.114 & 0.019 & 1.407 & $-0.059^{b}$ & -2.169 & -0.016 & -0.395 & -0.030 & -1.266 \\
\hline \multicolumn{11}{|c|}{ Panel D. North America Travel \& Leisure Index } \\
\hline OLS & $0.010^{\mathrm{a}}$ & 3.829 & $-0.016^{\mathrm{b}}$ & -2.498 & $-0.141^{\mathrm{a}}$ & -10.730 & -0.038 & -1.257 & $-0.032^{\mathrm{a}}$ & -3.270 \\
\hline 0.05 & $-0.068^{a}$ & -10.393 & $-0.041^{a}$ & -4.985 & $-0.148^{a}$ & -4.829 & 0.014 & 0.222 & $-0.047^{\mathrm{b}}$ & -2.409 \\
\hline 0.1 & $-0.038^{\mathrm{a}}$ & -8.159 & -0.013 & -1.055 & $-0.139^{\mathrm{a}}$ & -5.539 & -0.029 & -0.508 & $-0.072^{\mathrm{a}}$ & -4.155 \\
\hline 0.15 & $-0.029^{a}$ & -8.006 & -0.017 & -1.583 & $-0.153^{\mathrm{a}}$ & -6.634 & -0.076 & -1.603 & $-0.080^{\mathrm{a}}$ & -3.582 \\
\hline 0.2 & $-0.022^{\mathrm{a}}$ & -6.313 & $-0.021^{\mathrm{b}}$ & -2.207 & $-0.167^{\mathrm{a}}$ & -6.903 & -0.064 & -1.345 & -0.058 & -0.987 \\
\hline 0.25 & $-0.017^{\mathrm{a}}$ & -4.740 & $-0.025^{\mathrm{b}}$ & -2.490 & $-0.163^{a}$ & -6.506 & -0.065 & -1.404 & -0.064 & -0.892 \\
\hline 0.3 & $-0.010^{a}$ & -3.054 & $-0.018^{c}$ & -1.867 & $-0.152^{\mathrm{a}}$ & -6.342 & -0.074 & -1.675 & -0.044 & -1.205 \\
\hline 0.35 & -0.001 & -0.198 & -0.015 & -1.553 & $-0.131^{a}$ & -5.949 & -0.073 & -1.630 & $-0.041^{\mathrm{c}}$ & -1.923 \\
\hline 0.4 & 0.004 & 1.421 & -0.011 & -1.187 & $-0.126^{\mathrm{a}}$ & -6.109 & $-0.080^{c}$ & -1.838 & -0.031 & -1.517 \\
\hline 0.45 & $0.008^{a}$ & 2.692 & -0.011 & -1.182 & $-0.128^{a}$ & -6.388 & $-0.080^{c}$ & -1.871 & $-0.034^{\mathrm{c}}$ & -1.667 \\
\hline 0.5 & $0.011^{\mathrm{a}}$ & 4.041 & -0.005 & -0.572 & $-0.130^{\mathrm{a}}$ & -6.825 & $-0.081^{b}$ & -1.978 & $-0.033^{c}$ & -1.720 \\
\hline 0.55 & $0.015^{\mathrm{a}}$ & 5.203 & -0.004 & -0.517 & $-0.137^{\mathrm{a}}$ & -7.834 & $-0.075^{b}$ & -1.970 & -0.019 & -1.061 \\
\hline 0.6 & $0.020^{\mathrm{a}}$ & 7.127 & -0.006 & -0.646 & $-0.129^{a}$ & -8.379 & $-0.089^{b}$ & -2.529 & -0.020 & -1.251 \\
\hline 0.65 & $0.026^{\mathrm{a}}$ & 9.043 & -0.009 & -1.001 & $-0.130^{a}$ & -9.070 & $-0.080^{\mathrm{b}}$ & -2.281 & -0.019 & -1.143 \\
\hline 0.7 & $0.030^{\mathrm{a}}$ & 10.215 & -0.008 & -0.776 & $-0.123^{a}$ & -9.214 & -0.054 & -1.441 & -0.019 & -1.151 \\
\hline 0.75 & $0.036^{\mathrm{a}}$ & 11.527 & -0.013 & -1.263 & $-0.127^{a}$ & -9.244 & -0.030 & -0.829 & -0.008 & -0.584 \\
\hline 0.8 & $0.044^{\mathrm{a}}$ & 13.646 & -0.006 & -0.576 & $-0.124^{\mathrm{a}}$ & -8.983 & $-0.063^{\mathrm{c}}$ & -1.763 & -0.011 & -0.837 \\
\hline 0.85 & $0.049^{\mathrm{a}}$ & 14.938 & -0.004 & -0.335 & $-0.126^{a}$ & -9.581 & -0.048 & -1.438 & -0.013 & -1.161 \\
\hline 0.9 & $0.060^{\mathrm{a}}$ & 15.791 & -0.012 & -0.803 & $-0.126^{a}$ & -8.407 & 0.021 & 0.608 & 0.002 & 0.111 \\
\hline 0.95 & $0.074^{\mathrm{a}}$ & 16.327 & -0.008 & -0.468 & $-0.097^{\mathrm{a}}$ & -3.297 & 0.012 & 0.252 & -0.007 & -0.343 \\
\hline
\end{tabular}


Table 4. CLRM and QR models results (Geopolitical Acts)

Panel A. Global Travel \& Leisure Index

\begin{tabular}{|c|c|c|c|c|c|c|c|c|c|c|}
\hline & $\mathrm{c}$ & t-ratio & GPR-Acts & t-ratio & VIX & t-ratio & OIL & t-ratio & CRISIS & t-ratio \\
\hline OLS & $0.007^{\mathrm{a}}$ & 3.304 & $-0.014^{\mathrm{a}}$ & -3.268 & $-0.118^{a}$ & -10.248 & -0.017 & -0.640 & $-0.033^{\mathrm{a}}$ & -3.777 \\
\hline 0.05 & $-0.056^{\mathrm{a}}$ & -10.568 & $-0.023^{\mathrm{a}}$ & -3.546 & $-0.137^{a}$ & -5.313 & -0.016 & -0.182 & -0.040 & -0.735 \\
\hline 0.1 & $-0.042^{\mathrm{a}}$ & -5.538 & -0.016 & -1.011 & $-0.135^{\mathrm{a}}$ & -3.625 & 0.045 & 0.819 & -0.039 & -1.022 \\
\hline 0.15 & $-0.023^{\mathrm{a}}$ & -6.113 & $-0.016^{b}$ & -1.809 & $-0.146^{\mathrm{a}}$ & -5.282 & -0.033 & -0.528 & -0.054 & -1.244 \\
\hline 0.2 & $-0.018^{a}$ & -5.338 & $-0.011^{\mathrm{c}}$ & -1.704 & $-0.149^{a}$ & -7.079 & -0.052 & -1.265 & $-0.030^{\mathrm{b}}$ & -2.109 \\
\hline 0.25 & $-0.012^{\mathrm{a}}$ & -4.076 & $-0.012^{\mathrm{c}}$ & -1.961 & $-0.132^{a}$ & -6.751 & -0.034 & -0.855 & $-0.029^{a}$ & -2.735 \\
\hline 0.3 & $-0.006^{\mathrm{b}}$ & -2.221 & $-0.011^{\mathrm{b}}$ & -2.062 & $-0.126^{a}$ & -7.233 & $-0.075^{\mathrm{b}}$ & -2.176 & $-0.035^{\mathrm{a}}$ & -2.936 \\
\hline 0.35 & -0.001 & -0.583 & $-0.010^{b}$ & -2.044 & $-0.122^{\mathrm{a}}$ & -7.894 & $-0.082^{b}$ & -2.478 & $-0.036^{\mathrm{a}}$ & -3.114 \\
\hline 0.4 & 0.001 & 0.399 & $-0.011^{b}$ & -2.038 & $-0.124^{\mathrm{a}}$ & -8.445 & $-0.076^{b}$ & -2.267 & $-0.029^{a}$ & -2.734 \\
\hline 0.45 & $0.005^{\mathrm{b}}$ & 2.209 & $-0.011^{b}$ & -2.069 & $-0.119^{a}$ & -8.215 & -0.052 & -1.614 & $-0.034^{\mathrm{a}}$ & -3.300 \\
\hline 0.5 & $0.010^{\mathrm{a}}$ & 4.205 & $-0.016^{a}$ & -2.959 & $-0.121^{\mathrm{a}}$ & -8.429 & -0.045 & -1.463 & $-0.038^{a}$ & -3.921 \\
\hline 0.55 & $0.013^{\mathrm{a}}$ & 5.719 & $-0.015^{a}$ & -2.712 & $-0.114^{\mathrm{a}}$ & -8.799 & -0.049 & -1.536 & $-0.031^{\mathrm{a}}$ & -2.818 \\
\hline 0.6 & $0.017^{\mathrm{a}}$ & 7.408 & $-0.014^{b}$ & -2.340 & $-0.105^{\mathrm{a}}$ & -8.147 & -0.041 & -1.326 & $-0.033^{\mathrm{a}}$ & -2.793 \\
\hline 0.65 & $0.021^{\mathrm{a}}$ & 8.723 & $-0.012^{c}$ & -1.955 & $-0.109^{a}$ & -8.438 & -0.017 & -0.559 & $-0.033^{\mathrm{a}}$ & -2.894 \\
\hline 0.7 & $0.024^{\mathrm{a}}$ & 10.193 & -0.008 & -1.187 & $-0.105^{\mathrm{a}}$ & -8.164 & -0.030 & -0.979 & $-0.031^{\mathrm{a}}$ & -2.921 \\
\hline 0.75 & $0.029^{\mathrm{a}}$ & 11.600 & -0.005 & -0.721 & $-0.104^{a}$ & -8.044 & -0.017 & -0.491 & $-0.031^{\mathrm{a}}$ & -2.970 \\
\hline 0.8 & $0.033^{\mathrm{a}}$ & 12.807 & -0.006 & -0.945 & $-0.109^{a}$ & -8.940 & -0.013 & -0.384 & $-0.033^{\mathrm{a}}$ & -3.207 \\
\hline 0.85 & $0.043^{\mathrm{a}}$ & 13.271 & -0.007 & -0.894 & $-0.100^{\mathrm{a}}$ & -7.199 & 0.004 & 0.118 & $-0.030^{c}$ & -1.864 \\
\hline 0.9 & $0.053^{\mathrm{a}}$ & 13.036 & -0.004 & -0.474 & $-0.081^{\mathrm{a}}$ & -4.184 & -0.024 & -0.719 & $-0.026^{c}$ & -1.684 \\
\hline 0.95 & $0.062^{\mathrm{a}}$ & 16.966 & -0.001 & -0.067 & $-0.081^{\mathrm{a}}$ & -4.289 & 0.014 & 0.349 & -0.014 & -0.807 \\
\hline \multicolumn{11}{|c|}{ Panel B. Asia-Pacific Travel \& Leisure Index } \\
\hline OLS & 0.002 & 0.828 & -0.005 & -0.927 & $-0.091^{\mathrm{a}}$ & -6.400 & 0.007 & 0.218 & -0.018 & -1.630 \\
\hline 0.05 & $-0.076^{\mathrm{a}}$ & -10.644 & -0.001 & -0.093 & -0.079 & -1.515 & -0.113 & -0.692 & -0.049 & -1.011 \\
\hline 0.1 & $-0.056^{\mathrm{a}}$ & -9.029 & -0.015 & -1.273 & $-0.094^{b}$ & -2.130 & -0.040 & -0.488 & 0.003 & 0.163 \\
\hline 0.15 & $-0.039^{a}$ & -8.852 & -0.015 & -1.288 & $-0.074^{\mathrm{a}}$ & -3.383 & -0.001 & -0.018 & -0.013 & -0.858 \\
\hline 0.2 & $-0.032^{\mathrm{a}}$ & -7.177 & -0.009 & -1.026 & $-0.075^{\mathrm{a}}$ & -3.370 & 0.030 & 0.516 & -0.010 & -0.779 \\
\hline
\end{tabular}




\begin{tabular}{|c|c|c|c|c|c|c|c|c|c|c|}
\hline 0.25 & $-0.022^{a}$ & -5.962 & -0.006 & -0.790 & $-0.093^{\mathrm{a}}$ & -5.196 & 0.040 & 0.920 & -0.009 & -0.846 \\
\hline 0.3 & $-0.015^{\mathrm{a}}$ & -4.661 & -0.002 & -0.313 & $-0.081^{\mathrm{a}}$ & -6.164 & 0.042 & 1.097 & -0.014 & -1.313 \\
\hline 0.35 & $-0.009^{a}$ & -3.057 & -0.001 & -0.266 & $-0.093^{\mathrm{a}}$ & -7.873 & 0.014 & 0.386 & -0.019 & -1.628 \\
\hline 0.4 & $-0.005^{c}$ & -1.682 & 0.001 & 0.142 & $-0.094^{\mathrm{a}}$ & -8.214 & 0.008 & 0.219 & -0.018 & -1.493 \\
\hline 0.45 & -0.001 & -0.251 & -0.001 & -0.207 & $-0.088^{\mathrm{a}}$ & -7.554 & 0.028 & 0.762 & -0.012 & -1.094 \\
\hline 0.5 & 0.004 & 1.336 & 0.001 & 0.275 & $-0.093^{\mathrm{a}}$ & -7.990 & 0.018 & 0.519 & -0.011 & -1.071 \\
\hline 0.55 & $0.008^{\mathrm{a}}$ & 2.891 & 0.005 & 0.956 & $-0.096^{\mathrm{a}}$ & -7.789 & -0.003 & -0.090 & -0.013 & -1.226 \\
\hline 0.6 & $0.011^{\mathrm{a}}$ & 3.936 & 0.004 & 0.754 & $-0.092^{\mathrm{a}}$ & -6.859 & -0.004 & -0.108 & -0.016 & -1.561 \\
\hline 0.65 & $0.017^{\mathrm{a}}$ & 5.895 & 0.006 & 0.950 & $-0.092^{\mathrm{a}}$ & -5.557 & 0.014 & 0.357 & -0.011 & -1.030 \\
\hline 0.7 & $0.022^{\mathrm{a}}$ & 7.437 & 0.005 & 0.812 & $-0.092^{\mathrm{a}}$ & -4.756 & 0.005 & 0.123 & -0.013 & -1.277 \\
\hline 0.75 & $0.031^{\mathrm{a}}$ & 9.029 & 0.001 & 0.180 & $-0.101^{\mathrm{a}}$ & -4.254 & -0.020 & -0.516 & $-0.018^{b}$ & -1.973 \\
\hline 0.8 & $0.035^{\mathrm{a}}$ & 9.710 & 0.003 & 0.517 & $-0.099^{\mathrm{a}}$ & -3.547 & -0.037 & -0.927 & $-0.021^{b}$ & -2.436 \\
\hline 0.85 & $0.044^{\mathrm{a}}$ & 11.398 & 0.003 & 0.431 & $-0.076^{\mathrm{a}}$ & -2.832 & -0.047 & -1.374 & $-0.031^{\mathrm{a}}$ & -4.166 \\
\hline 0.9 & $0.056^{\mathrm{a}}$ & 11.888 & 0.011 & 1.138 & $-0.088^{\mathrm{a}}$ & -3.023 & -0.017 & -0.415 & $-0.032^{\mathrm{a}}$ & -3.363 \\
\hline 0.95 & $0.079^{\mathrm{a}}$ & 12.556 & -0.012 & -1.021 & $-0.082^{\mathrm{a}}$ & -2.932 & -0.010 & -0.278 & $-0.046^{a}$ & -3.944 \\
\hline \multicolumn{11}{|c|}{ Panel C. Europe Travel \& Leisure Index } \\
\hline OLS & $0.007^{\mathrm{a}}$ & 2.593 & $-0.016^{\mathrm{a}}$ & -3.080 & $-0.124^{\mathrm{a}}$ & -8.591 & $-0.080^{\mathrm{b}}$ & -2.459 & $-0.052^{\mathrm{a}}$ & -4.771 \\
\hline 0.05 & $-0.078^{\mathrm{a}}$ & -5.808 & -0.025 & -1.166 & $-0.223^{\mathrm{a}}$ & -4.012 & -0.159 & -1.643 & -0.095 & -1.302 \\
\hline 0.1 & $-0.051^{\mathrm{a}}$ & -7.235 & $-0.023^{\mathrm{c}}$ & -1.725 & $-0.155^{\mathrm{a}}$ & -5.021 & -0.090 & -0.713 & -0.076 & -1.281 \\
\hline 0.15 & $-0.034^{a}$ & -6.065 & -0.017 & -1.265 & $-0.150^{\mathrm{a}}$ & -4.634 & -0.067 & -0.950 & $-0.062^{\mathrm{a}}$ & -3.710 \\
\hline 0.2 & $-0.019^{a}$ & -5.420 & $-0.015^{\mathrm{c}}$ & -1.771 & $-0.123^{\mathrm{a}}$ & -6.365 & -0.070 & -1.356 & $-0.069^{a}$ & -3.685 \\
\hline 0.25 & $-0.016^{a}$ & -4.415 & -0.014 & -1.649 & $-0.130^{\mathrm{a}}$ & -6.455 & -0.071 & -1.360 & $-0.073^{\mathrm{a}}$ & -3.398 \\
\hline 0.3 & $-0.011^{\mathrm{a}}$ & -3.504 & -0.011 & -1.527 & $-0.125^{\mathrm{a}}$ & -7.616 & -0.079 & -1.607 & $-0.053^{\mathrm{a}}$ & -3.151 \\
\hline 0.35 & -0.004 & -1.408 & -0.009 & -1.317 & $-0.128^{\mathrm{a}}$ & -8.501 & $-0.103^{b}$ & -2.321 & $-0.057^{a}$ & -3.601 \\
\hline 0.4 & 0.001 & 0.257 & -0.005 & -0.886 & $-0.126^{\mathrm{a}}$ & -8.357 & $-0.119^{a}$ & -2.907 & $-0.060^{\mathrm{a}}$ & -4.174 \\
\hline 0.45 & $0.006^{\mathrm{b}}$ & 2.104 & -0.009 & -1.514 & $-0.124^{\mathrm{a}}$ & -7.888 & $-0.098^{b}$ & -2.494 & $-0.057^{\mathrm{a}}$ & -4.009 \\
\hline 0.5 & $0.010^{\mathrm{a}}$ & 3.316 & $-0.011^{\mathrm{c}}$ & -1.963 & $-0.120^{\mathrm{a}}$ & -7.644 & $-0.104^{\mathrm{a}}$ & -2.718 & $-0.056^{\mathrm{a}}$ & -4.128 \\
\hline 0.55 & $0.014^{\mathrm{a}}$ & 4.914 & $-0.013^{\mathrm{b}}$ & -2.134 & $-0.109^{a}$ & -7.455 & $-0.108^{a}$ & -2.912 & $-0.054^{\mathrm{a}}$ & -3.888 \\
\hline 0.6 & $0.019^{\mathrm{a}}$ & 6.519 & $-0.014^{\mathrm{b}}$ & -2.342 & $-0.099^{\mathrm{a}}$ & -6.617 & $-0.081^{b}$ & -2.322 & $-0.044^{\mathrm{a}}$ & -3.375 \\
\hline
\end{tabular}




\begin{tabular}{|c|c|c|c|c|c|c|c|c|c|c|}
\hline 0.65 & $0.023^{\mathrm{a}}$ & 8.132 & $-0.014^{\mathrm{b}}$ & -2.361 & $-0.100^{\mathrm{a}}$ & -6.652 & $-0.076^{b}$ & -2.170 & $-0.048^{\mathrm{a}}$ & -3.673 \\
\hline 0.7 & $0.027^{\mathrm{a}}$ & 9.637 & $-0.013^{\mathrm{b}}$ & -2.181 & $-0.095^{\mathrm{a}}$ & -6.198 & $-0.066^{c}$ & -1.881 & $-0.051^{\mathrm{a}}$ & -4.234 \\
\hline 0.75 & $0.035^{\mathrm{a}}$ & 11.477 & $-0.013^{\mathrm{b}}$ & -2.073 & $-0.080^{\mathrm{a}}$ & -3.952 & -0.059 & -1.603 & $-0.053^{\mathrm{a}}$ & -4.530 \\
\hline 0.8 & $0.040^{\mathrm{a}}$ & 12.853 & -0.009 & -1.403 & $-0.071^{\mathrm{a}}$ & -3.365 & $-0.057^{\mathrm{c}}$ & -1.666 & $-0.058^{\mathrm{a}}$ & -5.208 \\
\hline 0.85 & $0.048^{\mathrm{a}}$ & 14.464 & -0.009 & -1.093 & $-0.079^{\mathrm{a}}$ & -2.999 & -0.060 & -1.436 & $-0.050^{\mathrm{a}}$ & -2.634 \\
\hline 0.9 & $0.060^{\mathrm{a}}$ & 14.255 & -0.003 & -0.268 & $-0.073^{\mathrm{b}}$ & -2.519 & -0.017 & -0.362 & -0.040 & -1.565 \\
\hline 0.95 & $0.071^{\mathrm{a}}$ & 13.463 & 0.000 & -0.032 & -0.045 & -1.145 & -0.048 & -0.926 & -0.028 & -0.933 \\
\hline \multicolumn{11}{|c|}{ Panel D. North America Travel \& Leisure Index } \\
\hline OLS & $0.010^{\mathrm{a}}$ & 3.858 & $-0.015^{\mathrm{a}}$ & -3.167 & $-0.143^{\mathrm{a}}$ & -10.957 & -0.038 & -1.287 & $-0.032^{\mathrm{a}}$ & -3.291 \\
\hline 0.05 & $-0.063^{\mathrm{a}}$ & -8.743 & $-0.023^{\mathrm{b}}$ & -2.372 & $-0.167^{\mathrm{a}}$ & -5.372 & -0.030 & -0.269 & -0.056 & -1.508 \\
\hline 0.1 & $-0.044^{\mathrm{a}}$ & -7.811 & $-0.015^{\mathrm{c}}$ & -1.683 & $-0.173^{\mathrm{a}}$ & -5.355 & 0.015 & 0.237 & $-0.064^{\mathrm{a}}$ & -3.091 \\
\hline 0.15 & $-0.031^{\mathrm{a}}$ & -6.838 & $-0.014^{\mathrm{c}}$ & -1.772 & $-0.165^{\mathrm{a}}$ & -5.691 & -0.033 & -0.572 & $-0.062^{\mathrm{a}}$ & -2.949 \\
\hline 0.2 & $-0.024^{a}$ & -6.208 & -0.009 & -1.274 & $-0.166^{\mathrm{a}}$ & -6.530 & -0.063 & -1.389 & $-0.073^{\mathrm{a}}$ & -3.564 \\
\hline 0.25 & $-0.015^{\mathrm{a}}$ & -4.243 & $-0.014^{\mathrm{c}}$ & -1.768 & $-0.161^{a}$ & -6.398 & $-0.122^{b}$ & -2.535 & -0.068 & -1.624 \\
\hline 0.3 & $-0.009^{a}$ & -2.699 & -0.012 & -1.614 & $-0.159^{a}$ & -6.510 & $-0.081^{\mathrm{c}}$ & -1.675 & -0.044 & -1.242 \\
\hline 0.35 & -0.003 & -0.874 & -0.009 & -1.323 & $-0.139^{a}$ & -5.978 & -0.072 & -1.560 & $-0.037^{c}$ & -1.650 \\
\hline 0.4 & 0.004 & 1.357 & -0.009 & -1.468 & $-0.135^{\mathrm{a}}$ & -6.414 & $-0.073^{\mathrm{c}}$ & -1.663 & $-0.034^{\mathrm{c}}$ & -1.703 \\
\hline 0.45 & $0.008^{\mathrm{a}}$ & 2.776 & -0.008 & -1.298 & $-0.134^{\mathrm{a}}$ & -6.836 & $-0.085^{\mathrm{b}}$ & -2.114 & $-0.029^{c}$ & -1.668 \\
\hline 0.5 & $0.011^{\mathrm{a}}$ & 3.917 & -0.006 & -1.021 & $-0.134^{\mathrm{a}}$ & -7.020 & $-0.078^{c}$ & -1.931 & $-0.032^{\mathrm{c}}$ & -1.792 \\
\hline 0.55 & $0.016^{\mathrm{a}}$ & 5.540 & -0.008 & -1.422 & $-0.135^{\mathrm{a}}$ & -8.158 & $-0.083^{b}$ & -2.390 & -0.019 & -1.241 \\
\hline 0.6 & $0.020^{\mathrm{a}}$ & 7.139 & $-0.011^{\mathrm{c}}$ & -1.778 & $-0.132^{\mathrm{a}}$ & -8.519 & $-0.077^{\mathrm{b}}$ & -2.246 & -0.023 & -1.528 \\
\hline 0.65 & $0.025^{\mathrm{a}}$ & 8.858 & $-0.011^{\mathrm{c}}$ & -1.756 & $-0.130^{\mathrm{a}}$ & -9.269 & $-0.071^{\mathrm{b}}$ & -2.151 & -0.021 & -1.275 \\
\hline 0.7 & $0.030^{\mathrm{a}}$ & 10.442 & $-0.013^{b}$ & -2.081 & $-0.133^{\mathrm{a}}$ & -10.028 & $-0.073^{b}$ & -2.162 & $-0.027^{\mathrm{c}}$ & -1.721 \\
\hline 0.75 & $0.037^{\mathrm{a}}$ & 11.848 & $-0.01^{\mathrm{b}}$ & -2.144 & $-0.124^{\mathrm{a}}$ & -9.023 & -0.043 & -1.345 & -0.005 & -0.356 \\
\hline 0.8 & $0.042^{\mathrm{a}}$ & 13.176 & -0.010 & -1.378 & $-0.123^{\mathrm{a}}$ & -9.229 & -0.030 & -0.873 & -0.007 & -0.518 \\
\hline 0.85 & $0.050^{\mathrm{a}}$ & 14.777 & -0.011 & -1.384 & $-0.128^{\mathrm{a}}$ & -9.757 & -0.038 & -1.089 & -0.015 & -1.241 \\
\hline 0.9 & $0.060^{\mathrm{a}}$ & 16.468 & -0.010 & -1.104 & $-0.131^{\mathrm{a}}$ & -9.119 & 0.004 & 0.102 & -0.012 & -0.915 \\
\hline 0.95 & $0.073^{\mathrm{a}}$ & 17.110 & -0.010 & -0.972 & $-0.105^{\mathrm{a}}$ & -3.958 & 0.028 & 0.630 & -0.008 & -0.425 \\
\hline
\end{tabular}


Figure 3. Plots of quantile regression estimates (Geopolitical threats)

GLOBAL

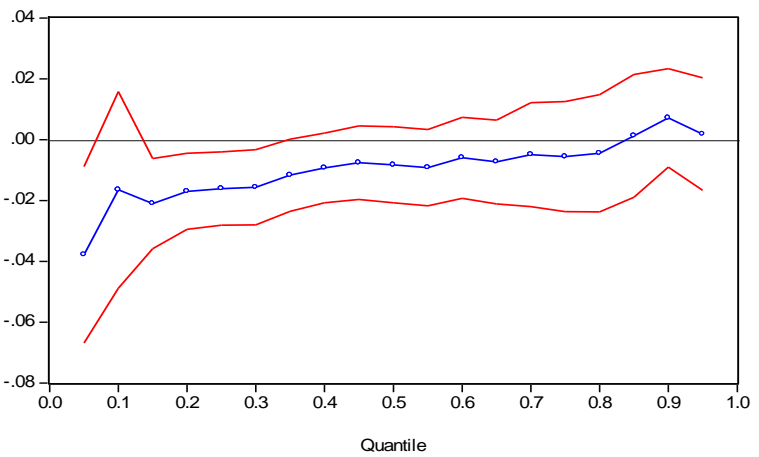

EUROPE

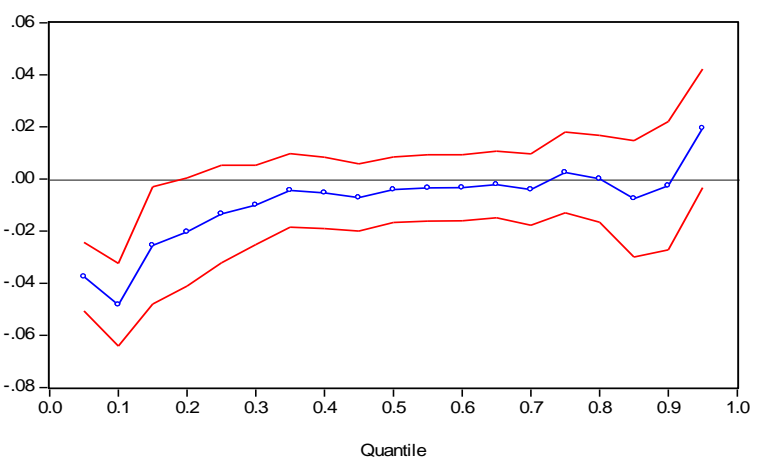

ASIA\&PACIFIC

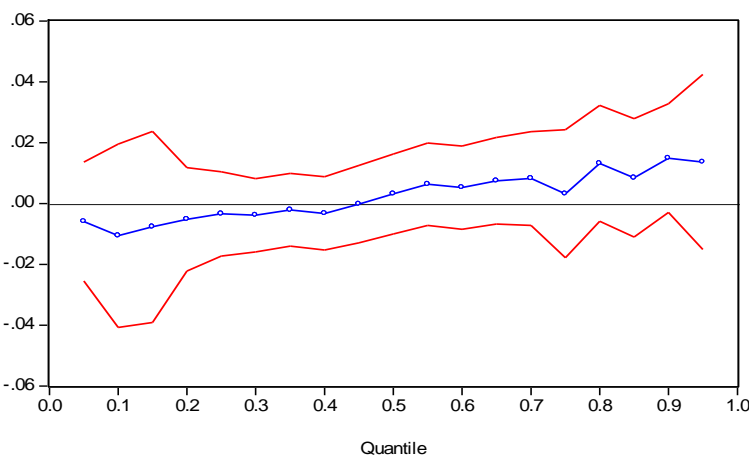

NORTH AMERICA

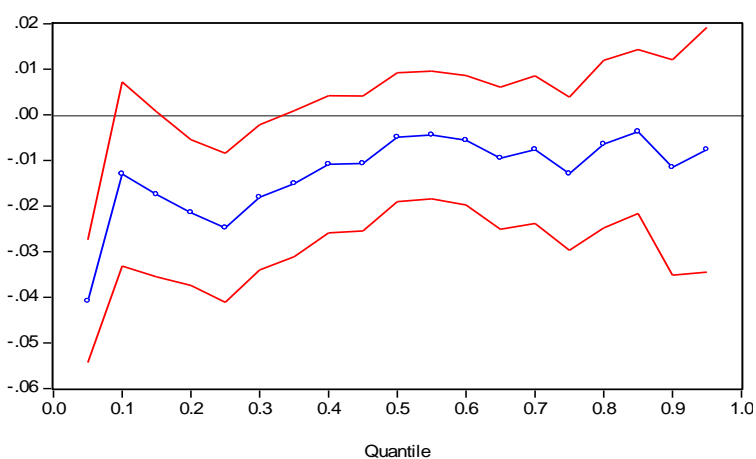

Figure 4. Plots of quantile regression estimates (Geopolitical acts)

GLOBAL

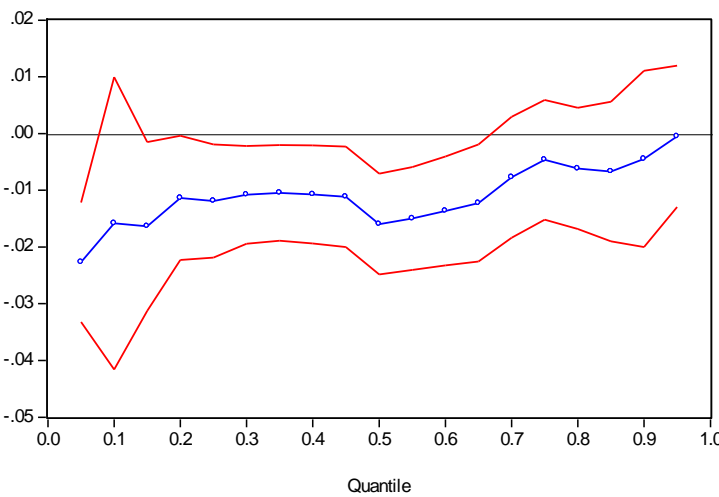

EUROPE

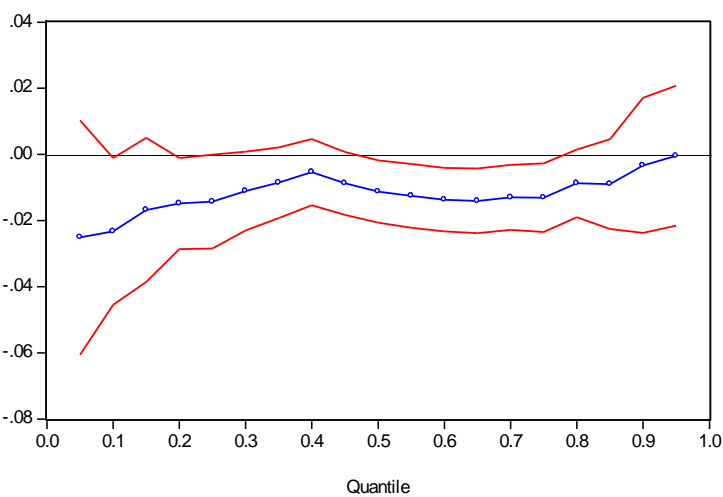

ASIA\&PACIFIC

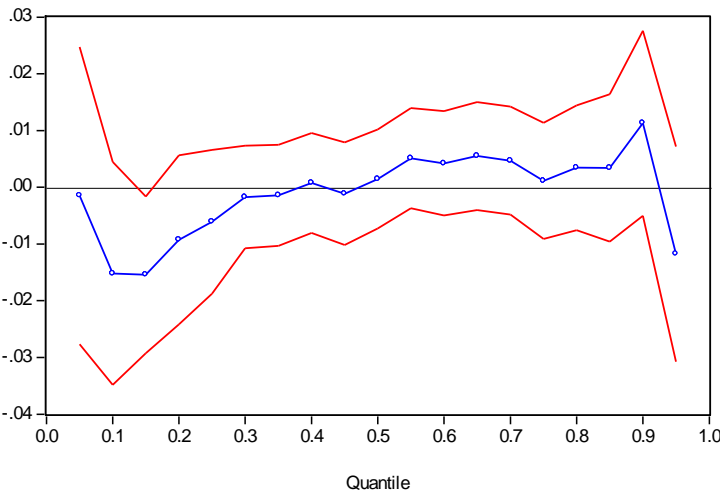

NORTH AMERICA

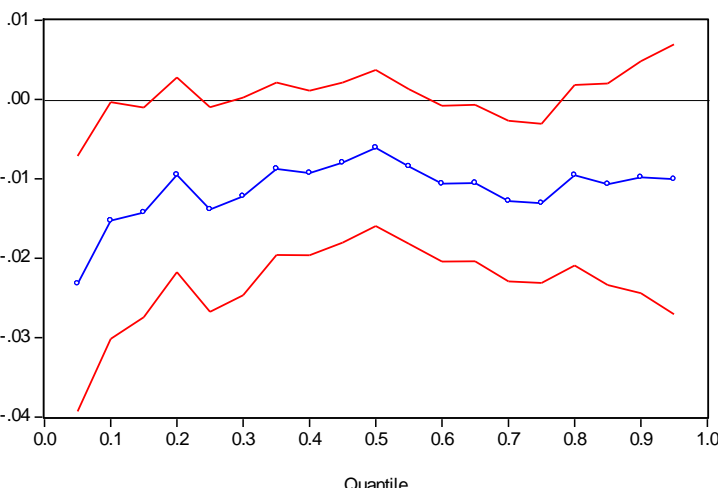




\subsection{The inclusion of economic policy uncertainty}

We also perform additional tests to check the robustness of our main findings. More specifically, we attempt to find out whether the above results hold after controlling for a potentially important omitted variable - namely, economic policy uncertainty (EPU) which refers to non-zero probability of economic policy change (Arouri et al., 2016). A very limited number of recent studies investigate the role of EPU in tourism industry and report that it has a predictive power for tourism stocks. In fact, EPU may significantly affect T\&L stock returns through similar channels to geopolitical risks. As suggested by Baker et al. (2016), a rise in EPU may make households and firms postpone consumption expenditure and investment. During the periods characterized by high uncertainty, demand for non-essential goods decreases and individuals can be reluctant to spend for holidays and recreational activities (Dragouni et al., 2016; Demir and Gozgor, 2018). A decrease or delay in tourism-related expenditures naturally translates into a decline in expected cash flows of T\&L companies, depressing their stock prices. Furthermore, when uncertainty is high, firms hold more cash and decrease their capital investments, which in turn leads to a higher cost of capital (Ersan et al., 2018).

We use U.S. EPU index as a proxy for global economic policy uncertainty. ${ }^{14}$ The EPU index developed by Baker et al. (2016) is a news-based index constructed from newspaper archives from the News Bank Access World News Database. ${ }^{15}$ The findings reported in Appendix 1, Appendix 2 and Appendix 3 show that EPU is an important determinant of the T\&L stock returns. The effect changes across quantiles and regions. Both the OLS and QR coefficients are negative, suggesting a rise in EPU results in a drop in index returns. The greater effect is found at lower and higher quantiles for the Global (over the quantile range of $\tau_{0.1}$ and and $\tau_{0.6}$ ) and Europe $T \& L$ indices (over the quantile range of $\tau_{0.05}$ and and $\tau_{0.55}$ ). The significant effects are found only at lower quantiles for the Asia\&Pacific $\left(\tau_{0.05}\right.$ and $\left.\tau_{0.1}\right)$ and North America indices ( $\tau_{0.1}$ and $\tau_{0.15}$ ). The negative impact of EPU is larger in magnitude for lower quantiles across all regions, providing evidence of asymmetric effects of policy uncertainty on T\&L indices. In other words, the negative effect of EPU is more pronounced at times T\&L stocks perform poor.

Overall, the results are similar after controlling for EPU, showing the robustness of our empirical findings. The empirical results from the regressions including EPU suggest that the effects of geopolitical risks on the Global, Europe and North American T\&L indices are more substantial during bear markets in which T\&L stock prices decline (see Appendix 1). The effects of geopolitical risks are mostly due to threats rather than acts; however, the impact is significant only for lower quantiles (see Appendix 2 and Appendix 3). The realization of events has significant effects at lower, intermediate and upper quantiles even if the effect is small. The results reveal insignificant effect for the Asia\&Pacific index. After re-running all regressions with the inclusion of EPU, we see that no qualitative result is changed by the variable. Therefore, the findings after controlling for EPU are consistent with the previous results.

\footnotetext{
${ }^{14}$ Baker et al. (2016) also constructed global EPU index. However, it is available since 1997. Due to data availability and given the fact that US economic variables partially reflect global ones, we use US EPU. We further check whether our results change using Global EPU and do the analyses accordingly. For this, the sample covers the period from January 1997 to August 2018. The empirical findings remain similar and are available upon request.

15 For more details about the methodology and construction of the index, visit the webpage at http://www.policyuncertainty.com/ or see Baker et al. (2016).
} 


\section{Discussions and Implications}

Although scholars have examined the effects of terrorism on tourism, the impact of geopolitical risks on T\&L companies' stock returns is still an untouched subject. Our study investigates this issue at regional level. After controlling for investor sentiment (VIX), oil prices and 2007-2009 global financial crisis, the results suggest negative impacts of geopolitical risks on the Global, Europe and North America T\&L stock returns. The findings from quantile regressions provide evidence of state dependence in the response of T\&L stock performance to geopolitical risks. The negative effects of geopolitical risks are more substantial at times T\&L sector performs poor.

Our findings do not provide any statistically significant evidence for the Asia\&Pacific region. It is worth noting that the Asia\&Pacific index includes Japan, Hong Kong, Australia, Singapore and New Zealand. Therefore, the index does not represent the whole region. Although our sample covers different terror attacks in the region, such as Bali bombings on 12 October 2002 and the bombing of the J. W. Marriott Hotel in Jakarta in August 2003, these incidences seem to have local consequences and do not have any adverse effect on stock returns of the largest $\mathrm{T} \& \mathrm{~L}$ companies in the region. However, these results provide support for the findings of some previous studies. A recent work of Zopiatis et al. (2018) shows the resilience of Asia\&Pacific hospitality and tourism stock index to terror events and wars. They attribute this to the region's isolated geopolitical location and the presence of local risks, which makes it less sensitive to external shocks. Donadelli (2015) also argues that Asian markets are mostly driven by domestic factors.

Our empirical results further reveal that the Global, Europe and North America T\&L indices are vulnerable to geopolitical risks. These risks can be viewed as exogenous factors leading to negative demand-side shocks. Heightened geopolitical risks can threaten the normal operating activity and conduct of tourism-related businesses. Ceteris paribus, an adverse tourism demand shock results in lower expected future earnings and dividends, depressing stock prices. For example, according to the International Air Transport Association, global air passenger traffic decreased by $17 \%$ in September 2001 following 9/11 terror attack compared to September 2000. In one week after the attack, airline stocks fell by $40 \%$ on average. The airline industry experienced net losses of more than $\$ 35$ billion from 2001 to 2004. A more recent example is the Paris attack for which the ISIS claimed responsibility, on November 13, 2015. Next day, $€ 2.3$ billion had been knocked off the value of T\&L stocks in Europe. Syrian war escalation and the emergence of the ISIS are the most important geopolitical risk factors for T\&L industry in the last years. The ISIS has not only created terror and tension in Iraq and Syria but also led to fear and social unrest globally (Stephens and Barbarani, 2016).

The general finding that geopolitical risks, including terrorism, political risks and wars have adverse effects on tourism-related industries' stocks is in line with the previous literature. Kim and $\mathrm{Gu}$ (2004) and Drakos (2004) find a substantial increase in idiosyncratic risk of airline stocks in the aftermath of 9/11. Chen et al (2005) documents significant impacts of the 9/11 attacks and 2003 Iraqi war on hotel stock returns. Chesney et al. (2011) show that stocks in airline, travel and tourism sectors are particularly sensitive to terrorist events. Examining the terrorist assaults in Paris on 13 November 2015 and the bombings in Brussels on 22 March 2016, Kolaric and Schiereck (2016) provide evidence of a strong short term impact on the valuation of airline companies. 
As known, the geopolitical risk index does not only capture terror events. It also includes other forms of geopolitical tensions such as war risk, military threats, nuclear tensions and geopolitical concerns. Therefore, it captures a broad array of exogenous global uncertainty (Balcilar et al., 2018). Tourism economics literature suggests that political instability (e.g., military coups, political violence, wars and religious/ethnic tensions) harms tourism industry. Our analysis is complementary to several papers quantifying the effects of political instability on tourism (Teye, 1988; Poirier, 1997; Neumayer, 2004; Issa and Altinay, 2006; Saha and Yap, 2014; Ivanov et al., 2017). Saha and Yap (2014) claims that social unrest as a result of military coups and wars leads to more prolonged damages to tourism than terrorism. As stated by Fletcher and Morakabati (2008), political instability is detrimental to tourism directly or indirectly. It can directly jeopardize a country's reputation and make the country unattractive to tourists by creating an image of lawlessness. It can also indirectly hinder the maintenance or development of tourism-specific infrastructure. In either case, the impact of political instability on T\&L stock returns is likely to be negative due to dropped number of overnights/tourists, declined demand for T\&L activities, increased costs and decreased company earnings.

Our empirical findings give evidence of asymmetric response of T\&L stock performance to geopolitical risks, suggesting that the negative impact of geopolitical events is more pronounced in times T\&L stock prices fall. When T\&L companies' earnings plummet under bearish market conditions, heightened geopolitical risks are more likely to change investors' predictions of the companies' expected cash flows. Bad news due to increasing geopolitical risks cause a downward revision in expected earnings and an upward revision in the required rate of return by investors. Therefore, the negative effect of geopolitical risks on T\&L companies' stock returns is expected to be stronger during episodes of falling stock prices. The state dependent nature of tourism-related stocks is documented in several studies. Chen (2011) empirically demonstrates that the reaction of hotel stock returns to international tourism development depends on the state of the economy. Ersan et al. (2018) recently find that economic policy uncertainty is a major determinant of European T\&L stock returns at bearish market times.

Moreover, our results suggest that the negative impacts of geopolitical risks are mostly driven by the threat of adverse geopolitical events rather than their realizations at times T\&L industry performs poor. However, we find strong negative response of T\&L stocks to geopolitical acts at all times. The results show limited effect of geopolitical threats, suggesting that militaryrelated tensions, war or terrorist threats lead to financial panic among investors in T\&L industry only when the industry is in bearish state. T\&L stock returns are sensitive to actual adverse geopolitical events, such as beginning of a war or terrorist acts, at all market states.

The findings in this paper provide significant insights and implications for investors who are contemplating T\&L stock investments, tourism authorities and managers. The Asia\&Pacific T\&L industry seems to be immune to geopolitical risks, indicating that the market can provide diversification benefits for stock investors. Overall, our findings provide evidence of sensitivity of T\&L stocks to geopolitical risks, especially to the realization of adverse geopolitical events. The results imply that, during periods characterized by high geopolitical risk, investors and other financial market players can flee from sensitive T\&L stocks to safer investments, such as government bonds, currencies and/or commodities, to protect themselves from losses. As for companies in T\&L industry, even if it is not possible to control the occurrence of geopolitical events, they should hedge against relevant risks with different strategies to minimize the adverse effects. Geopolitical conflicts have a profound influence on oil prices and tourism industry is 
highly oil-sensitive. T\&L companies can engage in oil futures trading to hedge against increasing oil prices. Results call for the necessity and importance of risk management to create strategies against unfavorable price movements.

As suggested by Sonmez (1999), tourism heavily depends on positive image of the destination. Given the fact that geopolitical risks can be detrimental to tourism and jeopardize the image, the industry must develop a recovery plan. For example, recovery marketing can be an option. Governments and tourism authorities should make strategic tourism plans to foster international travelling at times of high global geopolitical risks. Thus, they can contribute to the performance of T\&L companies through tourism development. All tourism stakeholders, including host governments, travel establishments, enterprises and associations need to cooperate in developing and operationalizing their crisis management plans to restore the destination image and mitigate the adverse effects of geopolitical risks.

\section{Conclusion and Future Research}

Previous literature in tourism research mostly focuses on the effects of terrorism on tourism demand or international tourism development. The impact of broader geopolitical risks is still an untouched subject. However, all stakeholders in tourism industry closely watch geopolitical risks to act accordingly. Understanding the industry reaction to geopolitical events is of paramount importance for tourism managers, investors and policy makers to mitigate the exposure and vulnerability and to develop recovery plans.

This study extends the relevant literature by exploring the effect of geopolitical risks on four T\&L stock indices returns. Given the fact that these indices cover the vast majority of the biggest T\&L companies around the world, our paper provides a broader perspective. By employing traditional and quantile regression methods, main empirical findings can be summarized as follows: i) We find a negative effect of geopolitical risks on T\&L stock index returns, except for the Asia \& Pacific index. ii) QR model shows the adverse impacts of geopolitical events are asymmetric, with a significant effect in lower quantiles. iii) The negative effects of geopolitical risks are mostly due to the threat of adverse geopolitical events rather than their realizations only at times $\mathrm{T} \& \mathrm{~L}$ industry performs poor. iv) We find strong negative response of T\&L stocks to geopolitical acts at all times, suggesting the realization of adverse geopolitical events significantly affect T\&L industry regardless of market state.

There are still several possible paths to follow in future studies to enhance our understanding of the effects of geopolitical risks on tourism industry. Future researchers can analyze the sensitivity of tourism industry to geopolitical events using company-level data from different countries to draw a more specific conclusion. This can also provide evidence at country-level. Furthermore, future studies can extend the investigation by distinguishing between short-run and long-run impacts. Such a distinction might give different implications for tourism stakeholders. 


\section{References}

Araña, J. E., \& León, C. J. (2008). The impact of terrorism on tourism demand. Annals of Tourism Research, 35(2), 299-315.

Arouri, M., Estay, C., Rault, C., \& Roubaud, D. (2016). Economic policy uncertainty and stock markets: Long-run evidence from the US. Finance Research Letters, 18, 136-141.

Assaf, A. G., \& Tsionas, M. (2018). Changing The Basics: Toward More Use of Quantile Regressions in Hospitality and Tourism Research. International Journal of Hospitality Management, 72, 140-144.

Baker, S. R., Bloom, N., \& Davis, S. J. (2016). Measuring economic policy uncertainty. The Quarterly Journal of Economics, 131(4), 1593-1636.

Balcilar, M., Bonato, M., Demirer, R., \& Gupta, R. (2018). Geopolitical risks and stock market dynamics of the BRICS. Economic Systems.

Bassil, C. (2014). The effect of terrorism on tourism demand in the Middle East. Peace Economics, Peace Science and Public Policy, 20(4), 669-684.

Brooks, C. (2013), Introductory Econometrics for Finance, University Press Cambridge

Buchinsky, M. (1995). Estimating the asymptotic covariance matrix for quantile regression models a Monte Carlo study. Journal of Econometrics, 68(2), 303-338.

Buigut, S., \& Amendah, D. D. (2016). Effect of terrorism on demand for tourism in Kenya. Tourism Economics, 22(5), 928-938.

Caldara, D., \& Iacoviello, M. (2018). Measuring geopolitical risk. Working Paper. Board of Governors of the Federal Reserve Board.

Chang, C., \& Zeng, Y. Y. (2011). Impact of terrorism on hospitality stocks and the role of investor sentiment. Cornell Hospitality Quarterly, 52(2), 165-175.

Chen, M. H. (2011). The response of hotel performance to international tourism development and crisis events. International Journal of Hospitality Management, 30(1), 200-212.

Chen, M. H., Kim, W. G., \& Kim, H. J. (2005). The impact of macroeconomic and nonmacroeconomic forces on hotel stock returns. International Journal of Hospitality Management, 24(2), 243-258.

Chen, R.J.C., \& Noriega P. (2004). The impacts of terrorism: perceptions of faculty and students on safety and security in Tourism. Journal of Tourism and Travel Marketing, 15 (23), 81-97.

Cheng, C. H. J., \& Chiu, C. W. J. (2018). How important are global geopolitical risks to emerging countries? International Economics.

Chesney, M., Reshetar, G., \& Karaman, M. (2011). The impact of terrorism on financial markets: An empirical study. Journal of Banking \& Finance, 35(2), 253-267.

Demir, E., \& Gozgor, G. (2018). Does economic policy uncertainty affect Tourism? Annals of Tourism Research, 69, 15-17. 
Donadelli, M. (2015). Asian stock markets, US economic policy uncertainty and US macroshocks. New Zealand Economic Papers, 49(2), 103-133.

Dragouni, M., Filis, G., Gavriilidis, K., \& Santamaria, D. (2016). Sentiment, mood and outbound tourism demand. Annals of Tourism Research, 60, 80-96.

Drakos, K. (2004). Terrorism-induced structural shifts in financial risk: airline stocks in the aftermath of the September 11th terror attacks. European Journal of Political Economy, 20(2), 435-446.

Drakos, K., \& Kutan, A. M. (2003). Regional effects of terrorism on tourism in three Mediterranean countries. Journal of Conflict Resolution, 47(5), 621-641.

Enders, W., \& Sandler, T. (1991). Causality between transnational terrorism and tourism: The case of Spain. Studies in Conflict \& Terrorism, 14(1), 49-58.

Enders, W., Sandler, T., \& Parise, G. F. (1992). An econometric analysis of the impact of terrorism on tourism. Kyklos, 45(4), 531-554.

Ersan, O., Akron, S., \& Demir, E. (2019). The effect of European and global uncertainty on stock returns of travel and leisure companies. Tourism Economics, 25(1), 51-66.

Fletcher, J., \& Morakabati, Y. (2008). Tourism activity, terrorism and political instability within the commonwealth: The cases of Fiji and Kenya. International Journal of Tourism Research, 10(6), 537-556.

Ioannides, D., \& Apostolopoulos, Y. (1999). Political instability, war, and tourism in Cyprus: Effects, management, and prospects for recovery. Journal of Travel Research, 38(1), 51-56.

Issa, I. A., \& Altinay, L. (2006). Impacts of political instability on tourism planning and development: the case of Lebanon. Tourism Economics, 12(3), 361-381.

Khan, A., \& Estrada, M. A. R. (2016). The effects of terrorism on economic performance: the case of Islamic State in Iraq and Syria (ISIS). Quality \& Quantity, 50(4), 1645-1661.

Kim, H., \& Gu, Z. (2004). Impact of the 9/11 terrorist attacks on the return and risk of airline stocks. Tourism and Hospitality Research, 5(2), 150-163.

Kim, H., \& Marcouiller, D. W. (2015). Considering disaster vulnerability and resiliency: the case of hurricane effects on tourism-based economies. The Annals of Regional Science, 54(3), 945-971.

Koenker, R., 2005. Quantile regression. Econometric Society Monograph Series. Cambridge University Press, New York.

Koenker, R., \& Bassett Jr, G. (1982). Robust tests for heteroscedasticity based on regression quantiles. Econometrica: Journal of the Econometric Society, 43-61.

Koenker, R., \& Machado, J. A. (1999). Goodness of fit and related inference processes for quantile regression. Journal of the American Statistical Association, 94(448), 1296-1310. 
Kolaric, S., \& Schiereck, D. (2016). Are stock markets efficient in the face of fear? Evidence from the terrorist attacks in Paris and Brussels. Finance Research Letters, 18, 306-310.

Kunreuther, H., \& Michel-Kerjan, E. (2004). Policy watch: challenges for terrorism risk insurance in the United States. Journal of Economic Perspectives, 18(4), 201-214.

Lanouar, C., \& Goaied, M. (2019). Tourism, terrorism and political violence in Tunisia: Evidence from Markov-switching models. Tourism Management, 70, 404-418.

Lintner, J. (1965). Security prices, risk, and maximal gains from diversification. The Journal of Finance, 20(4), 587-615.

Liu, A., \& Pratt, S. (2017). Tourism's vulnerability and resilience to terrorism. Tourism Management, 60, 404-417.

Madanoglu, M., Olsen, M. D., \& Kwansa, F. A. (2007). The impact of terrorist bombings on the market values of hospitality and tourism enterprises: Global evidence from Turkey, Spain, and Indonesia. The Journal of Hospitality Financial Management, 15(2), 49-60.

Marrocu, E., Paci, R., \& Zara, A. (2015). Micro-economic determinants of tourist expenditure: A quantile regression approach. Tourism Management, 50, 13-30.

Mensi, W., Hammoudeh, S., Reboredo, J. C., Nguyen, D. K. (2014). Do Global Factors Impact BRICS Stock Markets? A Quantile Regression Approach. Emerging Markets Review, 19, 117

Neumayer, E., 2004. The impact of political violence on tourism: Dynamic crosssectional estimation. J. Conflict Resolut. 48, 259-281

Pizam, A., \& Smith, G. (2000). Tourism and terrorism: A quantitative analysis of major terrorist acts and their impact on tourism destinations. Tourism Economics, 6(2), 123-138.

Poirier, R. A. (1997). Political risk analysis and tourism. Annals of Tourism Research, 24(3), 675-686.

Rao NV and Reddy KS (2015) The impact of the global financial crisis on cross-border mergers and acquisitions: a continental and industry analysis. Eurasian Business Review 5(2): 309-341.

Rittichainuwat, B. N., \& Chakraborty, G. (2009). Perceived travel risks regarding terrorism and disease: The case of Thailand. Tourism Management, 30(3), 410-418.

Ross, S. A., Westerfield, R., \& Jordan, B. D. (2008). Fundamentals of corporate finance. Tata McGraw-Hill Education.

Saha, S., \& Yap, G. (2014). The moderation effects of political instability and terrorism on tourism development: A cross-country panel analysis. Journal of Travel Research, 53(4), 509521.

Samatas, M. (2007). Security and surveillance in the Athens 2004 Olympics: Some lessons from a troubled story. International Criminal Justice Review, 17(3), 220-238.

Sandler, T., \& Enders, W. (2008). Economic consequences of terrorism in developed and developing countries. Terrorism, economic development, and political openness, 17. 
Seetanah, B. (2011). Assessing the dynamic economic impact of tourism for island economies. Annals of Tourism Research, 38(1), 291-308.

Seraphin, H. (2017). Terrorism and tourism in France: the limitations of dark tourism. Worldwide Hospitality and Tourism Themes, 9(2), 187-195.

Sönmez, S. F. (1998). Tourism, terrorism, and political instability. Annals of Tourism Research, 25(2), 416-456.

Sönmez, S. F., Apostolopoulos, Y., \& Tarlow, P. (1999). Tourism in crisis: Managing the effects of terrorism. Journal of Travel Research, 38(1), 13-18.

Sönmez, S. F., Apostolopoulos, Y., \& Tarlow, P. (1999). Tourism in crisis: Managing the effects of terrorism. Journal of Travel Research, 38(1), 13-18.

Teye, V. B. (1988). Coups d'etat and African tourism: A study of Ghana. Annals of Tourism Research, 15(3), 329-356.

Wang, Y. S. (2013). The impact of crisis events on the stock returns volatility of international airlines. The Service Industries Journal, 33(12), 1206-1217.

Wearden, G., and K. Allen. 2015. "Billions Wiped off European Travel Shares after Paris Attacks." The Guardian, November 16. Available at: <https://www.theguardian.com/business/2015/nov/16/billions-wiped-off-european-travelshares-after-paris-attacks>

Zopiatis, A., Savva, C. S., Lambertides, N., \& McAleer, M. (2017). Tourism Stocks in Times of Crisis: An Econometric Investigation of Unexpected Nonmacroeconomic Factors. Journal of Travel Research, 0047287517753998. 
Appendix 1. CLRM and QR models results including EPU (Geopolitical Risks)

Panel A. Global Travel \& Leisure Index

\begin{tabular}{|c|c|c|c|c|c|c|c|c|c|c|c|c|}
\hline & $\mathrm{c}$ & t-ratio & GPR & t-ratio & VIX & t-ratio & EPU & t-ratio & OIL & t-ratio & CRISIS & t-ratio \\
\hline OLS & $0.007^{\mathrm{a}}$ & 3.260 & $-0.016^{\mathrm{a}}$ & -2.747 & $-0.110^{\mathrm{a}}$ & -9.423 & $-0.036^{a}$ & -2.810 & -0.018 & -0.688 & $-0.031^{\mathrm{a}}$ & -3.600 \\
\hline 0.05 & $-0.056^{\mathrm{a}}$ & -7.891 & $-0.039^{a}$ & -2.763 & $-0.145^{\mathrm{a}}$ & -4.186 & -0.040 & -1.065 & -0.006 & -0.078 & -0.056 & -1.638 \\
\hline 0.1 & $-0.039^{\mathrm{a}}$ & -8.720 & $-0.031^{\mathrm{a}}$ & -3.162 & $-0.154^{\mathrm{a}}$ & -6.578 & $-0.080^{\mathrm{a}}$ & -3.827 & 0.004 & 0.073 & $-0.055^{\mathrm{b}}$ & -2.083 \\
\hline 0.15 & $-0.027^{\mathrm{a}}$ & -6.369 & $-0.021^{\mathrm{c}}$ & -1.930 & $-0.139^{a}$ & -6.727 & $-0.074^{\mathrm{a}}$ & -3.674 & -0.036 & -0.757 & -0.052 & -1.640 \\
\hline 0.2 & $-0.019^{a}$ & -5.576 & $-0.017^{b}$ & -2.013 & $-0.123^{a}$ & -7.424 & $-0.055^{\mathrm{a}}$ & -2.763 & -0.005 & -0.124 & $-0.031^{b}$ & -2.284 \\
\hline 0.25 & $-0.013^{\mathrm{a}}$ & -3.947 & -0.013 & -1.412 & $-0.119^{a}$ & -7.427 & $-0.042^{b}$ & -2.099 & -0.027 & -0.719 & $-0.032^{b}$ & -2.589 \\
\hline 0.3 & $-0.008^{a}$ & -2.934 & -0.012 & -1.510 & $-0.123^{a}$ & -8.014 & $-0.044^{a}$ & -2.629 & -0.049 & -1.281 & $-0.025^{\mathrm{a}}$ & -2.658 \\
\hline 0.35 & -0.002 & -0.683 & $-0.015^{b}$ & -2.008 & $-0.114^{\mathrm{a}}$ & -7.672 & $-0.032^{\mathrm{b}}$ & -2.488 & $-0.065^{b}$ & -2.074 & $-0.028^{\mathrm{a}}$ & -2.851 \\
\hline 0.4 & 0.000 & 0.158 & $-0.013^{\mathrm{c}}$ & -1.704 & $-0.116^{\mathrm{a}}$ & -7.655 & $-0.030^{b}$ & -2.381 & $-0.065^{\mathrm{b}}$ & -2.072 & $-0.030^{\mathrm{a}}$ & -2.897 \\
\hline 0.45 & $0.004^{\mathrm{c}}$ & 1.739 & -0.012 & -1.566 & $-0.112^{\mathrm{a}}$ & -7.435 & $-0.030^{b}$ & -2.346 & $-0.053^{c}$ & -1.674 & $-0.034^{\mathrm{a}}$ & -3.173 \\
\hline 0.5 & $0.008^{a}$ & 3.375 & -0.011 & -1.450 & $-0.110^{\mathrm{a}}$ & -7.817 & $-0.023^{\mathrm{c}}$ & -1.948 & $-0.064^{b}$ & -2.135 & $-0.028^{a}$ & -2.711 \\
\hline 0.55 & $0.012^{\mathrm{a}}$ & 5.091 & -0.010 & -1.304 & $-0.101^{\mathrm{a}}$ & -7.633 & $-0.029^{b}$ & -2.263 & $-0.055^{c}$ & -1.838 & $-0.029^{a}$ & -2.747 \\
\hline 0.6 & $0.017^{\mathrm{a}}$ & 6.976 & -0.012 & -1.442 & $-0.102^{\mathrm{a}}$ & -7.803 & $-0.029^{b}$ & -2.124 & $-0.053^{c}$ & -1.730 & $-0.033^{\mathrm{a}}$ & -3.156 \\
\hline 0.65 & $0.022^{\mathrm{a}}$ & 8.794 & -0.012 & -1.401 & $-0.103^{\mathrm{a}}$ & -8.358 & -0.020 & -1.471 & -0.037 & -1.170 & $-0.035^{\mathrm{a}}$ & -3.196 \\
\hline 0.7 & $0.026^{\mathrm{a}}$ & 10.191 & -0.006 & -0.593 & $-0.103^{\mathrm{a}}$ & -7.957 & -0.016 & -1.032 & -0.041 & -1.169 & $-0.030^{b}$ & -2.459 \\
\hline 0.75 & $0.029^{\mathrm{a}}$ & 11.211 & -0.006 & -0.554 & $-0.103^{a}$ & -8.284 & -0.016 & -0.936 & -0.016 & -0.468 & $-0.029^{b}$ & -2.176 \\
\hline 0.8 & $0.033^{\mathrm{a}}$ & 12.283 & -0.006 & -0.465 & $-0.101^{a}$ & -8.494 & -0.022 & -1.233 & 0.000 & -0.014 & $-0.030^{b}$ & -2.302 \\
\hline 0.85 & $0.040^{\mathrm{a}}$ & 12.938 & 0.003 & 0.231 & $-0.098^{\mathrm{a}}$ & -6.406 & -0.022 & -1.055 & -0.036 & -1.358 & -0.015 & -1.129 \\
\hline 0.9 & $0.051^{\mathrm{a}}$ & 13.771 & 0.006 & 0.540 & $-0.082^{\mathrm{a}}$ & -4.215 & -0.022 & -0.811 & -0.015 & -0.496 & $-0.024^{\mathrm{c}}$ & -1.941 \\
\hline 0.95 & $0.063^{\mathrm{a}}$ & 15.773 & 0.004 & 0.388 & $-0.067^{\mathrm{a}}$ & -3.159 & -0.018 & -0.741 & -0.007 & -0.177 & -0.020 & -1.139 \\
\hline \multicolumn{13}{|c|}{ Panel B. Asia-Pacific Travel \& Leisure Index } \\
\hline OLS & 0.002 & 0.794 & -0.006 & -0.819 & $-0.084^{\mathrm{a}}$ & -5.822 & $-0.035^{\mathrm{b}}$ & -2.200 & 0.006 & 0.194 & -0.016 & -1.483 \\
\hline 0.05 & $-0.082^{\mathrm{a}}$ & -9.692 & -0.004 & -0.274 & $-0.129^{\mathrm{a}}$ & -2.600 & $-0.040^{\mathrm{b}}$ & -2.028 & 0.039 & 0.183 & 0.010 & 0.428 \\
\hline 0.1 & $-0.056^{\mathrm{a}}$ & -9.809 & -0.013 & -0.670 & $-0.059^{c}$ & -1.768 & $-0.042^{\mathrm{b}}$ & -2.313 & -0.030 & -0.352 & -0.015 & -0.557 \\
\hline 0.15 & $-0.040^{\mathrm{a}}$ & -8.511 & -0.024 & -1.422 & $-0.066^{\mathrm{b}}$ & -2.514 & -0.014 & -0.582 & 0.018 & 0.258 & -0.015 & -0.809 \\
\hline 0.2 & $-0.029^{a}$ & -7.081 & -0.006 & -0.482 & $-0.075^{\mathrm{a}}$ & -3.283 & -0.010 & -0.441 & 0.028 & 0.504 & -0.013 & -0.952 \\
\hline
\end{tabular}




\begin{tabular}{|c|c|c|c|c|c|c|c|c|c|c|c|c|}
\hline 0.25 & $-0.020^{\mathrm{a}}$ & -5.746 & -0.012 & -1.298 & $-0.087^{\mathrm{a}}$ & -5.012 & -0.024 & -0.905 & 0.036 & 0.840 & -0.012 & -0.950 \\
\hline 0.3 & $-0.015^{\mathrm{a}}$ & -4.848 & -0.009 & -1.094 & $-0.097^{\mathrm{a}}$ & -7.064 & -0.022 & -1.052 & 0.011 & 0.294 & -0.011 & -0.934 \\
\hline 0.35 & $-0.010^{\mathrm{a}}$ & -3.398 & -0.005 & -0.620 & $-0.091^{\mathrm{a}}$ & -7.823 & -0.020 & -1.067 & 0.000 & 0.008 & -0.012 & -0.904 \\
\hline 0.4 & $-0.005^{\mathrm{c}}$ & -1.652 & 0.001 & 0.148 & $-0.092^{\mathrm{a}}$ & -7.720 & -0.025 & -1.304 & 0.000 & 0.003 & -0.016 & -1.142 \\
\hline 0.45 & -0.001 & -0.221 & 0.000 & 0.058 & $-0.093^{\mathrm{a}}$ & -7.911 & -0.018 & -0.990 & 0.029 & 0.809 & -0.015 & -1.189 \\
\hline 0.5 & 0.003 & 1.101 & 0.000 & 0.027 & $-0.089^{a}$ & -7.432 & -0.023 & -1.285 & 0.026 & 0.715 & -0.011 & -0.920 \\
\hline 0.55 & $0.008^{a}$ & 2.801 & 0.006 & 0.719 & $-0.085^{\mathrm{a}}$ & -6.346 & -0.020 & -1.145 & -0.010 & -0.301 & -0.009 & -0.755 \\
\hline 0.6 & $0.012^{\mathrm{a}}$ & 4.174 & 0.004 & 0.493 & $-0.087^{\mathrm{a}}$ & -5.964 & -0.023 & -1.256 & -0.018 & -0.567 & -0.011 & -1.005 \\
\hline 0.65 & $0.018^{a}$ & 6.077 & 0.009 & 0.908 & $-0.086^{\mathrm{a}}$ & -4.926 & -0.024 & -1.276 & 0.003 & 0.091 & -0.012 & -1.117 \\
\hline 0.7 & $0.023^{\mathrm{a}}$ & 7.720 & 0.008 & 0.768 & $-0.088^{\mathrm{a}}$ & -4.772 & -0.027 & -1.313 & 0.004 & 0.133 & -0.004 & -0.427 \\
\hline 0.75 & $0.028^{a}$ & 8.863 & 0.005 & 0.441 & $-0.088^{a}$ & -4.053 & -0.030 & -1.552 & -0.007 & -0.200 & -0.011 & -1.124 \\
\hline 0.8 & $0.035^{\mathrm{a}}$ & 9.511 & 0.011 & 0.855 & $-0.088^{\mathrm{a}}$ & -3.562 & -0.028 & -1.387 & -0.018 & -0.415 & -0.016 & -1.506 \\
\hline 0.85 & $0.044^{\mathrm{a}}$ & 10.615 & 0.012 & 1.031 & $-0.074^{\mathrm{a}}$ & -2.595 & -0.012 & -0.470 & -0.025 & -0.621 & $-0.021^{\mathrm{b}}$ & -2.065 \\
\hline 0.9 & $0.055^{\mathrm{a}}$ & 11.932 & 0.015 & 1.280 & $-0.075^{\mathrm{a}}$ & -2.603 & -0.011 & -0.427 & -0.026 & -0.694 & $-0.032^{\mathrm{a}}$ & -3.609 \\
\hline 0.95 & $0.077^{\mathrm{a}}$ & 11.035 & 0.011 & 0.563 & $-0.073^{\mathrm{b}}$ & -2.098 & -0.013 & -0.439 & -0.019 & -0.606 & $-0.053^{\mathrm{a}}$ & -5.673 \\
\hline \multicolumn{13}{|c|}{ Panel C. Europe Travel \& Leisure Index } \\
\hline OLS & $0.007^{\mathrm{a}}$ & 2.567 & $-0.016^{\mathrm{b}}$ & -2.155 & $-0.110^{\mathrm{a}}$ & -7.629 & $-0.068^{\mathrm{a}}$ & -4.273 & $-0.082^{\mathrm{b}}$ & -2.571 & $-0.049^{\mathrm{a}}$ & -4.555 \\
\hline 0.05 & $-0.067^{\mathrm{a}}$ & -10.095 & $-0.025^{\mathrm{b}}$ & -2.358 & $-0.176^{\mathrm{a}}$ & -8.532 & $-0.117^{\mathrm{a}}$ & -3.412 & $-0.151^{\mathrm{c}}$ & -1.891 & $-0.076^{c}$ & -1.777 \\
\hline 0.1 & $-0.047^{\mathrm{a}}$ & -10.528 & $-0.041^{\mathrm{a}}$ & -3.665 & $-0.143^{\mathrm{a}}$ & -6.501 & $-0.116^{\mathrm{a}}$ & -6.336 & -0.024 & -0.579 & $-0.046^{\mathrm{a}}$ & -3.473 \\
\hline 0.15 & $-0.036^{\mathrm{a}}$ & -7.227 & -0.017 & -0.955 & $-0.124^{\mathrm{a}}$ & -5.857 & $-0.108^{\mathrm{a}}$ & -5.625 & -0.061 & -1.219 & $-0.049^{\mathrm{a}}$ & -3.539 \\
\hline 0.2 & $-0.025^{\mathrm{a}}$ & -6.624 & -0.003 & -0.256 & $-0.119^{a}$ & -6.576 & $-0.085^{\mathrm{a}}$ & -4.265 & $-0.099^{c}$ & -1.981 & $-0.053^{\mathrm{a}}$ & -3.881 \\
\hline 0.25 & $-0.018^{\mathrm{a}}$ & -4.973 & -0.007 & -0.706 & $-0.124^{\mathrm{a}}$ & -6.711 & $-0.076^{\mathrm{a}}$ & -3.847 & $-0.119^{\mathrm{a}}$ & -2.927 & $-0.056^{\mathrm{a}}$ & -4.226 \\
\hline 0.3 & $-0.012^{\mathrm{a}}$ & -3.451 & -0.015 & -1.440 & $-0.106^{\mathrm{a}}$ & -6.484 & $-0.067^{\mathrm{a}}$ & -3.435 & $-0.110^{\mathrm{a}}$ & -2.697 & $-0.059^{\mathrm{a}}$ & -3.989 \\
\hline 0.35 & $-0.008^{b}$ & -2.291 & -0.010 & -1.039 & $-0.115^{\mathrm{a}}$ & -6.818 & $-0.056^{\mathrm{a}}$ & -2.833 & $-0.118^{\mathrm{a}}$ & -3.002 & $-0.056^{\mathrm{a}}$ & -3.939 \\
\hline 0.4 & 0.000 & -0.159 & -0.014 & -1.615 & $-0.105^{\mathrm{a}}$ & -7.201 & $-0.047^{\mathrm{a}}$ & -2.810 & $-0.105^{\mathrm{a}}$ & -2.810 & $-0.048^{\mathrm{a}}$ & -3.760 \\
\hline 0.45 & 0.004 & 1.335 & -0.008 & -0.992 & $-0.109^{\mathrm{a}}$ & -7.452 & $-0.041^{\mathrm{b}}$ & -2.501 & $-0.095^{\mathrm{b}}$ & -2.568 & $-0.049^{\mathrm{a}}$ & -3.735 \\
\hline 0.5 & $0.009^{\mathrm{a}}$ & 2.857 & -0.005 & -0.618 & $-0.102^{\mathrm{a}}$ & -7.327 & $-0.034^{\mathrm{b}}$ & -2.110 & $-0.100^{\mathrm{a}}$ & -2.714 & $-0.052^{\mathrm{a}}$ & -3.900 \\
\hline 0.55 & $0.013^{\mathrm{a}}$ & 4.179 & -0.007 & -0.888 & $-0.103^{\mathrm{a}}$ & -7.217 & $-0.031^{\mathrm{b}}$ & -1.933 & $-0.087^{b}$ & -2.390 & $-0.052^{\mathrm{a}}$ & -4.105 \\
\hline 0.6 & $0.017^{\mathrm{a}}$ & 5.421 & -0.006 & -0.731 & $-0.092^{\mathrm{a}}$ & -6.240 & -0.027 & -1.605 & $-0.085^{\mathrm{b}}$ & -2.364 & $-0.054^{\mathrm{a}}$ & -4.179 \\
\hline
\end{tabular}




\begin{tabular}{|c|c|c|c|c|c|c|c|c|c|c|c|c|}
\hline 0.65 & $0.023^{\mathrm{a}}$ & 7.089 & -0.005 & -0.606 & $-0.092^{\mathrm{a}}$ & -5.555 & -0.015 & -0.832 & -0.057 & -1.497 & $-0.049^{a}$ & -3.758 \\
\hline 0.7 & $0.028^{\mathrm{a}}$ & 8.849 & -0.010 & -1.081 & $-0.095^{\mathrm{a}}$ & -5.532 & -0.014 & -0.739 & -0.048 & -1.254 & $-0.052^{a}$ & -4.062 \\
\hline 0.75 & $0.035^{\mathrm{a}}$ & 10.796 & -0.003 & -0.294 & $-0.073^{\mathrm{a}}$ & -3.259 & -0.001 & -0.027 & -0.056 & -1.519 & $-0.056^{\mathrm{a}}$ & -4.558 \\
\hline 0.8 & $0.042^{\mathrm{a}}$ & 12.985 & -0.001 & -0.125 & $-0.062^{\mathrm{a}}$ & -2.711 & -0.015 & -0.621 & $-0.066^{c}$ & -1.770 & $-0.056^{\mathrm{a}}$ & -4.155 \\
\hline 0.85 & $0.050^{\mathrm{a}}$ & 14.863 & 0.000 & 0.006 & $-0.077^{\mathrm{a}}$ & -2.939 & -0.032 & -0.954 & -0.056 & -1.161 & $-0.048^{\mathrm{b}}$ & -2.363 \\
\hline 0.9 & $0.058^{\mathrm{a}}$ & 15.686 & 0.001 & 0.056 & $-0.075^{\mathrm{a}}$ & -2.885 & -0.008 & -0.222 & -0.024 & -0.542 & -0.041 & -1.630 \\
\hline 0.95 & $0.072^{\mathrm{a}}$ & 12.587 & 0.011 & 0.632 & -0.036 & -0.750 & -0.025 & -0.469 & -0.056 & -0.862 & -0.026 & -1.053 \\
\hline \multicolumn{13}{|c|}{ Panel D. North America Travel \& Leisure Index } \\
\hline OLS & $0.009^{\mathrm{a}}$ & 3.831 & $-0.019^{\mathrm{a}}$ & -2.782 & $-0.140^{\mathrm{a}}$ & -10.440 & -0.008 & -0.562 & -0.037 & -1.240 & $-0.032^{\mathrm{a}}$ & -3.239 \\
\hline 0.05 & $-0.066^{\mathrm{a}}$ & -10.138 & $-0.040^{\mathrm{a}}$ & -2.620 & $-0.147^{\mathrm{a}}$ & -5.109 & -0.018 & -1.185 & 0.020 & 0.318 & $-0.046^{b}$ & -2.372 \\
\hline 0.1 & $-0.044^{\mathrm{a}}$ & -5.752 & -0.017 & -1.135 & $-0.153^{\mathrm{a}}$ & -4.302 & $-0.034^{\mathrm{a}}$ & -2.688 & -0.016 & -0.255 & $-0.056^{\mathrm{b}}$ & -2.488 \\
\hline 0.15 & $-0.029^{a}$ & -7.890 & $-0.020^{c}$ & -1.803 & $-0.147^{\mathrm{a}}$ & -6.130 & $-0.028^{b}$ & -2.227 & -0.053 & -1.112 & $-0.079^{a}$ & -4.160 \\
\hline 0.2 & $-0.023^{\mathrm{a}}$ & -6.304 & $-0.021^{\mathrm{c}}$ & -1.951 & $-0.163^{a}$ & -6.915 & 0.003 & 0.113 & -0.051 & -1.136 & $-0.082^{\mathrm{a}}$ & -3.584 \\
\hline 0.25 & $-0.017^{\mathrm{a}}$ & -4.710 & $-0.026^{\mathrm{b}}$ & -2.386 & $-0.166^{\mathrm{a}}$ & -6.523 & -0.002 & -0.094 & -0.075 & -1.590 & -0.062 & -1.073 \\
\hline 0.3 & $-0.009^{\mathrm{a}}$ & -2.779 & $-0.020^{c}$ & -1.942 & $-0.149^{\mathrm{a}}$ & -6.718 & -0.015 & -0.693 & -0.074 & -1.759 & -0.044 & -1.243 \\
\hline 0.35 & -0.001 & -0.374 & -0.016 & -1.505 & $-0.127^{\mathrm{a}}$ & -6.086 & -0.017 & -0.959 & -0.069 & -1.618 & $-0.036^{c}$ & -1.896 \\
\hline 0.4 & 0.003 & 1.027 & -0.014 & -1.341 & $-0.129^{a}$ & -6.283 & -0.015 & -0.836 & -0.062 & -1.470 & $-0.035^{\mathrm{c}}$ & -1.820 \\
\hline 0.45 & $0.008^{a}$ & 2.800 & -0.008 & -0.827 & $-0.127^{\mathrm{a}}$ & -6.544 & -0.009 & -0.455 & $-0.080^{c}$ & -1.905 & -0.032 & -1.612 \\
\hline 0.5 & $0.011^{\mathrm{a}}$ & 3.801 & -0.008 & -0.823 & $-0.132^{\mathrm{a}}$ & -6.863 & -0.009 & -0.477 & $-0.087^{\mathrm{b}}$ & -2.116 & $-0.033^{c}$ & -1.789 \\
\hline 0.55 & $0.014^{\mathrm{a}}$ & 5.085 & -0.005 & -0.503 & $-0.136^{\mathrm{a}}$ & -7.888 & -0.002 & -0.116 & $-0.079^{\mathrm{b}}$ & -2.127 & -0.018 & -1.076 \\
\hline 0.6 & $0.020^{\mathrm{a}}$ & 7.228 & -0.007 & -0.737 & $-0.128^{a}$ & -8.627 & 0.001 & 0.044 & $-0.096^{\mathrm{a}}$ & -2.781 & -0.020 & -1.297 \\
\hline 0.65 & $0.025^{\mathrm{a}}$ & 8.741 & -0.013 & -1.232 & $-0.136^{\mathrm{a}}$ & -9.006 & 0.003 & 0.193 & $-0.075^{\mathrm{b}}$ & -2.134 & -0.018 & -1.113 \\
\hline 0.7 & $0.030^{\mathrm{a}}$ & 10.184 & -0.008 & -0.746 & $-0.122^{\mathrm{a}}$ & -8.709 & 0.001 & 0.058 & -0.053 & -1.423 & -0.020 & -1.131 \\
\hline 0.75 & $0.036^{\mathrm{a}}$ & 11.503 & -0.012 & -1.105 & $-0.128^{a}$ & -8.805 & 0.006 & 0.303 & -0.036 & -1.025 & -0.008 & -0.538 \\
\hline 0.8 & $0.044^{\mathrm{a}}$ & 13.444 & -0.010 & -0.849 & $-0.125^{\mathrm{a}}$ & -8.343 & -0.004 & -0.199 & -0.049 & -1.361 & -0.010 & -0.695 \\
\hline 0.85 & $0.051^{\mathrm{a}}$ & 14.624 & -0.006 & -0.502 & $-0.131^{\mathrm{a}}$ & -8.022 & 0.007 & 0.373 & -0.049 & -1.362 & -0.015 & -1.136 \\
\hline 0.9 & $0.059^{\mathrm{a}}$ & 16.319 & -0.013 & -0.911 & $-0.132^{\mathrm{a}}$ & -7.179 & 0.007 & 0.335 & 0.001 & 0.015 & -0.006 & -0.308 \\
\hline 0.95 & $0.074^{\mathrm{a}}$ & 16.913 & -0.015 & -1.062 & $-0.100^{\mathrm{a}}$ & -3.404 & -0.003 & -0.155 & 0.014 & 0.291 & -0.005 & -0.305 \\
\hline
\end{tabular}


Appendix 2. CLRM and QR models results including EPU (Geopolitical Threats)

Panel A. Global Travel \& Leisure Index

\begin{tabular}{|c|c|c|c|c|c|c|c|c|c|c|c|c|}
\hline & $\mathrm{c}$ & & GPR-THRE & & VIX & & EPU & & OIL & & CRISIS & \\
\hline OLS & $0.007^{\mathrm{a}}$ & 3.239 & $-0.013^{b}$ & -2.372 & $-0.110^{\mathrm{a}}$ & -9.373 & $-0.037^{a}$ & -2.869 & -0.019 & -0.723 & $-0.031^{\mathrm{a}}$ & -3.570 \\
\hline 0.05 & $-0.065^{\mathrm{a}}$ & -6.091 & -0.020 & -0.663 & $-0.150^{\mathrm{a}}$ & -3.716 & -0.078 & -0.864 & 0.030 & 0.340 & -0.029 & -0.861 \\
\hline 0.1 & $-0.040^{\mathrm{a}}$ & -8.230 & $-0.030^{\mathrm{a}}$ & -3.168 & $-0.155^{\mathrm{a}}$ & -6.221 & $-0.080^{\mathrm{a}}$ & -3.687 & 0.009 & 0.172 & $-0.053^{b}$ & -2.299 \\
\hline 0.15 & $-0.026^{a}$ & -6.793 & $-0.019^{b}$ & -1.987 & $-0.137^{\mathrm{a}}$ & -7.232 & $-0.070^{a}$ & -3.539 & -0.031 & -0.638 & $-0.058^{c}$ & -1.937 \\
\hline 0.2 & $-0.019^{a}$ & -5.684 & $-0.017^{b}$ & -2.070 & $-0.122^{\mathrm{a}}$ & -7.600 & $-0.054^{\mathrm{a}}$ & -2.785 & 0.000 & 0.007 & $-0.030^{\mathrm{b}}$ & -2.462 \\
\hline 0.25 & $-0.012^{\mathrm{a}}$ & -3.876 & $-0.016^{b}$ & -1.883 & $-0.117^{\mathrm{a}}$ & -7.328 & $-0.040^{a}$ & -2.158 & -0.030 & -0.817 & $-0.033^{\mathrm{a}}$ & -2.621 \\
\hline 0.3 & $-0.008^{a}$ & -2.883 & -0.010 & -1.292 & $-0.121^{a}$ & -7.735 & $-0.043^{a}$ & -2.660 & -0.054 & -1.401 & $-0.024^{\mathrm{b}}$ & -2.513 \\
\hline 0.35 & -0.003 & -1.032 & -0.005 & -0.712 & $-0.115^{\mathrm{a}}$ & -7.197 & $-0.032^{b}$ & -2.507 & $-0.070^{\mathrm{b}}$ & -2.100 & $-0.025^{\mathrm{b}}$ & -2.448 \\
\hline 0.4 & 0.001 & 0.362 & -0.007 & -1.125 & $-0.115^{\mathrm{a}}$ & -7.482 & $-0.030^{\mathrm{b}}$ & -2.399 & $-0.068^{b}$ & -2.145 & $-0.029^{a}$ & -2.769 \\
\hline 0.45 & $0.004^{\mathrm{c}}$ & 1.665 & -0.008 & -1.093 & $-0.110^{\mathrm{a}}$ & -7.347 & $-0.029^{b}$ & -2.355 & $-0.055^{c}$ & -1.731 & $-0.033^{a}$ & -3.004 \\
\hline 0.5 & $0.009^{\mathrm{a}}$ & 3.591 & -0.010 & -1.318 & $-0.108^{a}$ & -7.667 & $-0.023^{c}$ & -1.911 & $-0.058^{c}$ & -1.886 & $-0.029^{a}$ & -2.780 \\
\hline 0.55 & $0.013^{\mathrm{a}}$ & 5.264 & -0.010 & -1.407 & $-0.098^{a}$ & -7.356 & $-0.032^{b}$ & -2.436 & $-0.054^{c}$ & -1.802 & $-0.029^{a}$ & -2.764 \\
\hline 0.6 & $0.016^{\mathrm{a}}$ & 6.811 & -0.007 & -0.967 & $-0.099^{a}$ & -7.576 & $-0.029^{b}$ & -2.139 & -0.051 & -1.640 & $-0.033^{a}$ & -3.137 \\
\hline 0.65 & $0.021^{\mathrm{a}}$ & 8.677 & -0.009 & -1.066 & $-0.106^{\mathrm{a}}$ & -8.330 & -0.020 & -1.516 & -0.042 & -1.301 & $-0.035^{\mathrm{a}}$ & -3.447 \\
\hline 0.7 & $0.025^{\mathrm{a}}$ & 10.104 & -0.004 & -0.356 & $-0.103^{a}$ & -7.818 & -0.018 & -1.109 & -0.050 & -1.389 & $-0.028^{b}$ & -2.171 \\
\hline 0.75 & $0.029^{\mathrm{a}}$ & 11.231 & -0.004 & -0.354 & $-0.102^{a}$ & -8.270 & -0.019 & -1.060 & -0.017 & -0.473 & $-0.029^{b}$ & -2.150 \\
\hline 0.8 & $0.034^{\mathrm{a}}$ & 11.989 & 0.001 & 0.056 & $-0.097^{\mathrm{a}}$ & -7.545 & -0.026 & -1.329 & -0.008 & -0.220 & -0.027 & -1.634 \\
\hline 0.85 & $0.040^{\mathrm{a}}$ & 13.143 & 0.003 & 0.262 & $-0.098^{\mathrm{a}}$ & -6.512 & -0.021 & -1.009 & -0.035 & -1.376 & -0.015 & -1.128 \\
\hline 0.9 & $0.051^{\mathrm{a}}$ & 13.688 & 0.006 & 0.580 & $-0.083^{\mathrm{a}}$ & -4.082 & -0.019 & -0.626 & -0.014 & -0.463 & $-0.024^{c}$ & -1.909 \\
\hline 0.95 & $0.063^{\mathrm{a}}$ & 16.155 & 0.004 & 0.354 & $-0.070^{\mathrm{a}}$ & -3.328 & -0.016 & -0.659 & -0.005 & -0.111 & -0.019 & -1.046 \\
\hline \multicolumn{13}{|c|}{ Panel B. Asia-Pacific Travel \& Leisure Index } \\
\hline OLS & 0.002 & 0.791 & -0.005 & -0.656 & $-0.084^{\mathrm{a}}$ & -5.814 & $-0.035^{b}$ & -2.225 & 0.006 & 0.182 & -0.016 & -1.475 \\
\hline 0.05 & $-0.082^{\mathrm{a}}$ & -9.150 & -0.003 & -0.273 & $-0.128^{b}$ & -2.245 & $-0.079^{c}$ & -1.790 & 0.035 & 0.151 & -0.025 & -0.543 \\
\hline 0.1 & $-0.056^{a}$ & -10.033 & -0.008 & -0.492 & $-0.056^{c}$ & -1.748 & $-0.087^{b}$ & -2.125 & -0.031 & -0.360 & -0.016 & -0.562 \\
\hline 0.15 & $-0.038^{a}$ & -7.795 & -0.004 & -0.229 & $-0.064^{b}$ & -2.497 & -0.011 & -0.405 & 0.027 & 0.343 & -0.013 & -0.617 \\
\hline 0.2 & $-0.029^{a}$ & -6.907 & -0.004 & -0.316 & $-0.080^{a}$ & -3.200 & -0.023 & -0.757 & 0.018 & 0.316 & -0.012 & -0.926 \\
\hline
\end{tabular}




\begin{tabular}{|c|c|c|c|c|c|c|c|c|c|c|c|c|}
\hline 0.25 & $-0.020^{a}$ & -5.847 & -0.010 & -1.281 & $-0.088^{a}$ & -5.157 & -0.024 & -0.942 & 0.030 & 0.689 & -0.021 & -1.492 \\
\hline 0.3 & $-0.015^{\mathrm{a}}$ & -4.904 & -0.008 & -1.071 & $-0.096^{\mathrm{a}}$ & -7.233 & -0.021 & -1.026 & 0.002 & 0.059 & -0.014 & -1.069 \\
\hline 0.35 & $-0.009^{a}$ & -3.158 & -0.004 & -0.507 & $-0.094^{\mathrm{a}}$ & -8.087 & -0.024 & -1.274 & -0.011 & -0.301 & -0.017 & -1.202 \\
\hline 0.4 & $-0.005^{\mathrm{c}}$ & -1.693 & 0.001 & 0.123 & $-0.092^{\mathrm{a}}$ & -7.718 & -0.026 & -1.324 & 0.000 & 0.007 & -0.019 & -1.426 \\
\hline 0.45 & -0.001 & -0.224 & 0.000 & 0.062 & $-0.093^{a}$ & -7.890 & -0.018 & -0.996 & 0.029 & 0.821 & -0.015 & -1.190 \\
\hline 0.5 & 0.004 & 1.344 & 0.002 & 0.315 & $-0.091^{\mathrm{a}}$ & -7.565 & -0.023 & -1.319 & 0.020 & 0.561 & -0.011 & -0.901 \\
\hline 0.55 & $0.008^{\mathrm{a}}$ & 2.743 & 0.006 & 0.746 & $-0.082^{\mathrm{a}}$ & -6.033 & -0.023 & -1.341 & -0.008 & -0.213 & -0.009 & -0.764 \\
\hline 0.6 & $0.012^{\mathrm{a}}$ & 4.180 & 0.004 & 0.546 & $-0.088^{a}$ & -6.207 & -0.021 & -1.170 & -0.014 & -0.424 & -0.010 & -0.930 \\
\hline 0.65 & $0.017^{\mathrm{a}}$ & 6.099 & 0.006 & 0.703 & $-0.088^{a}$ & -5.098 & -0.026 & -1.368 & 0.004 & 0.122 & -0.010 & -1.016 \\
\hline 0.7 & $0.024^{\mathrm{a}}$ & 7.807 & 0.006 & 0.578 & $-0.089^{a}$ & -4.560 & -0.036 & -1.844 & -0.002 & -0.052 & -0.010 & -1.107 \\
\hline 0.75 & $0.028^{a}$ & 8.831 & 0.004 & 0.376 & $-0.089^{a}$ & -4.010 & -0.029 & -1.484 & -0.008 & -0.229 & -0.011 & -1.117 \\
\hline 0.8 & $0.035^{\mathrm{a}}$ & 9.452 & 0.011 & 0.913 & $-0.088^{a}$ & -3.611 & -0.032 & -1.567 & -0.011 & -0.252 & -0.015 & -1.314 \\
\hline 0.85 & $0.043^{\mathrm{a}}$ & 10.330 & 0.012 & 0.985 & $-0.076^{a}$ & -2.635 & -0.014 & -0.504 & -0.018 & -0.425 & $-0.021^{b}$ & -1.979 \\
\hline 0.9 & $0.055^{\mathrm{a}}$ & 11.873 & 0.014 & 1.187 & $-0.078^{a}$ & -2.719 & -0.011 & -0.412 & -0.030 & -0.802 & $-0.032^{\mathrm{a}}$ & -3.761 \\
\hline 0.95 & $0.078^{\mathrm{a}}$ & 11.258 & 0.012 & 0.654 & $-0.070^{b}$ & -2.168 & -0.008 & -0.263 & -0.030 & -0.920 & $-0.055^{\mathrm{a}}$ & -5.954 \\
\hline \multicolumn{13}{|c|}{ Panel C. Europe Travel \& Leisure Index } \\
\hline OLS & $0.007^{\mathrm{b}}$ & 2.555 & $-0.012^{\mathrm{c}}$ & -1.794 & $-0.110^{\mathrm{a}}$ & -7.596 & $-0.068^{\mathrm{a}}$ & -4.323 & $-0.083^{\mathrm{a}}$ & -2.595 & $-0.048^{\mathrm{a}}$ & -4.529 \\
\hline 0.05 & $-0.070^{\mathrm{a}}$ & -11.152 & $-0.031^{\mathrm{b}}$ & -2.494 & $-0.183^{a}$ & -8.932 & $-0.135^{\mathrm{a}}$ & -4.891 & $-0.186^{\mathrm{b}}$ & -2.237 & $-0.091^{\mathrm{b}}$ & -2.143 \\
\hline 0.1 & $-0.048^{a}$ & -10.160 & $-0.021^{\mathrm{b}}$ & -1.998 & $-0.144^{\mathrm{a}}$ & -6.280 & $-0.124^{\mathrm{a}}$ & -6.672 & -0.021 & -0.484 & $-0.043^{\mathrm{a}}$ & -3.199 \\
\hline 0.15 & $-0.037^{\mathrm{a}}$ & -7.773 & $-0.018^{c}$ & -1.808 & $-0.126^{\mathrm{a}}$ & -5.932 & $-0.107^{\mathrm{a}}$ & -5.483 & -0.072 & -1.471 & $-0.057^{\mathrm{a}}$ & -3.317 \\
\hline 0.2 & $-0.025^{a}$ & -6.646 & -0.003 & -0.279 & $-0.119^{a}$ & -6.567 & $-0.085^{\mathrm{a}}$ & -4.277 & $-0.097^{\mathrm{c}}$ & -1.919 & $-0.053^{a}$ & -3.836 \\
\hline 0.25 & $-0.019^{a}$ & -5.168 & -0.004 & -0.398 & $-0.124^{\mathrm{a}}$ & -6.697 & $-0.081^{\mathrm{a}}$ & -4.032 & $-0.130^{\mathrm{a}}$ & -3.272 & $-0.054^{\mathrm{a}}$ & -4.225 \\
\hline 0.3 & $-0.012^{a}$ & -3.524 & -0.008 & -0.917 & $-0.101^{\mathrm{a}}$ & -6.240 & $-0.065^{\mathrm{a}}$ & -3.298 & $-0.111^{\mathrm{a}}$ & -2.695 & $-0.056^{\mathrm{a}}$ & -3.826 \\
\hline 0.35 & $-0.006^{c}$ & -1.947 & -0.006 & -0.706 & $-0.114^{\mathrm{a}}$ & -6.923 & $-0.054^{\mathrm{a}}$ & -2.812 & $-0.123^{a}$ & -3.180 & $-0.059^{\mathrm{a}}$ & -4.038 \\
\hline 0.4 & -0.001 & -0.352 & -0.009 & -1.044 & $-0.108^{a}$ & -7.337 & $-0.047^{\mathrm{a}}$ & -2.760 & $-0.116^{\mathrm{a}}$ & -3.097 & $-0.052^{\mathrm{a}}$ & -3.932 \\
\hline 0.45 & 0.004 & 1.372 & -0.007 & -0.926 & $-0.109^{a}$ & -7.387 & $-0.040^{b}$ & -2.440 & $-0.095^{b}$ & -2.543 & $-0.049^{\mathrm{a}}$ & -3.775 \\
\hline 0.5 & $0.009^{\mathrm{a}}$ & 3.133 & -0.001 & -0.166 & $-0.104^{\mathrm{a}}$ & -7.325 & $-0.034^{\mathrm{b}}$ & -2.173 & $-0.099^{a}$ & -2.690 & $-0.053^{\mathrm{a}}$ & -4.007 \\
\hline 0.55 & $0.013^{\mathrm{a}}$ & 4.161 & -0.006 & -0.744 & $-0.105^{a}$ & -7.121 & $-0.031^{\mathrm{c}}$ & -1.910 & $-0.082^{b}$ & -2.231 & $-0.051^{\mathrm{a}}$ & -4.116 \\
\hline 0.6 & $0.017^{\mathrm{a}}$ & 5.294 & -0.005 & -0.576 & $-0.093^{\mathrm{a}}$ & -6.404 & $-0.028^{c}$ & -1.707 & $-0.082^{b}$ & -2.291 & $-0.053^{\mathrm{a}}$ & -4.214 \\
\hline
\end{tabular}




\begin{tabular}{|c|c|c|c|c|c|c|c|c|c|c|c|c|}
\hline 0.65 & $0.023^{\mathrm{a}}$ & 6.926 & 0.000 & -0.023 & $-0.093^{\mathrm{a}}$ & -5.717 & -0.015 & -0.811 & $-0.071^{\mathrm{c}}$ & -1.912 & $-0.053^{a}$ & -4.217 \\
\hline 0.7 & $0.029^{\mathrm{a}}$ & 9.028 & -0.007 & -0.860 & $-0.090^{\mathrm{a}}$ & -5.110 & -0.018 & -0.949 & $-0.047^{c}$ & -1.219 & $-0.053^{a}$ & -4.113 \\
\hline 0.75 & $0.037^{\mathrm{a}}$ & 11.123 & 0.003 & 0.273 & $-0.066^{\mathrm{a}}$ & -2.887 & -0.002 & -0.073 & $-0.068^{c}$ & -1.803 & $-0.054^{a}$ & -4.087 \\
\hline 0.8 & $0.042^{\mathrm{a}}$ & 12.939 & 0.001 & 0.051 & $-0.064^{\mathrm{a}}$ & -2.729 & -0.012 & -0.470 & $-0.072^{\mathrm{c}}$ & -1.686 & $-0.042^{b}$ & -2.328 \\
\hline 0.85 & $0.049^{\mathrm{a}}$ & 15.025 & 0.001 & 0.045 & $-0.077^{\mathrm{a}}$ & -2.970 & -0.030 & -0.919 & -0.057 & -1.197 & $-0.048^{b}$ & -2.352 \\
\hline 0.9 & $0.057^{\mathrm{a}}$ & 16.248 & 0.002 & 0.156 & $-0.078^{a}$ & -3.087 & -0.012 & -0.355 & -0.032 & -0.714 & $-0.043^{c}$ & -1.702 \\
\hline 0.95 & $0.073^{\mathrm{a}}$ & 14.701 & 0.021 & 1.514 & $-0.057^{c}$ & -1.764 & -0.001 & -0.036 & -0.010 & -0.229 & -0.030 & -1.266 \\
\hline \multicolumn{13}{|c|}{ Panel D. North America Travel \& Leisure Index } \\
\hline OLS & $0.009^{\mathrm{a}}$ & 3.813 & $-0.016^{\mathrm{b}}$ & -2.420 & $-0.140^{\mathrm{a}}$ & -10.385 & -0.009 & -0.622 & -0.038 & -1.270 & $-0.032^{\mathrm{a}}$ & -3.213 \\
\hline 0.05 & $-0.067^{\text {a }}$ & -10.040 & $-0.037^{\mathrm{b}}$ & -2.208 & $-0.155^{\mathrm{a}}$ & -5.179 & $-0.028^{\mathrm{b}}$ & -2.347 & 0.019 & 0.300 & $-0.045^{\mathrm{b}}$ & -2.260 \\
\hline 0.1 & $-0.044^{\mathrm{a}}$ & -6.117 & -0.013 & -0.864 & $-0.145^{\mathrm{a}}$ & -4.033 & $-0.023^{\mathrm{b}}$ & -2.171 & -0.014 & -0.235 & $-0.059^{\mathrm{a}}$ & -2.661 \\
\hline 0.15 & $-0.029^{a}$ & -8.070 & -0.017 & -1.599 & $-0.152^{a}$ & -6.634 & -0.018 & -1.605 & -0.076 & -1.604 & $-0.077^{\mathrm{a}}$ & -3.703 \\
\hline 0.2 & $-0.022^{a}$ & -6.367 & $-0.023^{\mathrm{b}}$ & -2.340 & $-0.167^{\mathrm{a}}$ & -7.001 & -0.013 & -1.123 & -0.073 & -1.522 & -0.057 & -0.884 \\
\hline 0.25 & $-0.017^{\mathrm{a}}$ & -4.816 & $-0.024^{b}$ & -2.415 & $-0.167^{\mathrm{a}}$ & -6.561 & -0.009 & -1.060 & -0.058 & -1.252 & -0.063 & -0.932 \\
\hline 0.3 & $-0.010^{a}$ & -2.960 & $-0.018^{\mathrm{c}}$ & -1.904 & $-0.149^{\mathrm{a}}$ & -6.441 & -0.006 & -0.918 & $-0.078^{\mathrm{c}}$ & -1.790 & -0.044 & -1.176 \\
\hline 0.35 & -0.002 & -0.569 & -0.015 & -1.493 & $-0.128^{a}$ & -6.080 & -0.009 & -1.043 & $-0.073^{c}$ & -1.722 & $-0.035^{\mathrm{c}}$ & -1.808 \\
\hline 0.4 & 0.004 & 1.288 & -0.012 & -1.270 & $-0.129^{a}$ & -6.339 & -0.015 & -1.432 & $-0.071^{\mathrm{c}}$ & -1.687 & $-0.034^{\mathrm{c}}$ & -1.758 \\
\hline 0.45 & $0.008^{\mathrm{a}}$ & 2.732 & -0.005 & -0.556 & $-0.128^{a}$ & -6.609 & -0.013 & -1.117 & $-0.082^{b}$ & -1.972 & $-0.033^{c}$ & -1.678 \\
\hline 0.5 & $0.011^{\mathrm{a}}$ & 3.787 & -0.004 & -0.525 & $-0.134^{\mathrm{a}}$ & -6.949 & -0.008 & -0.809 & $-0.088^{b}$ & -2.171 & $-0.032^{\mathrm{c}}$ & -1.796 \\
\hline 0.55 & $0.014^{\mathrm{a}}$ & 5.057 & -0.004 & -0.494 & $-0.135^{\mathrm{a}}$ & -7.857 & -0.002 & -0.100 & $-0.079^{b}$ & -2.129 & -0.018 & -1.044 \\
\hline 0.6 & $0.020^{\mathrm{a}}$ & 7.051 & -0.006 & -0.650 & $-0.129^{a}$ & -8.360 & 0.000 & -0.028 & $-0.088^{b}$ & -2.510 & -0.020 & -1.241 \\
\hline 0.65 & $0.026^{\mathrm{a}}$ & 8.839 & -0.009 & -0.984 & $-0.132^{\mathrm{a}}$ & -9.067 & 0.002 & 0.097 & $-0.083^{b}$ & -2.381 & -0.019 & -1.142 \\
\hline 0.7 & $0.030^{\mathrm{a}}$ & 10.132 & -0.008 & -0.828 & $-0.123^{\mathrm{a}}$ & -8.736 & 0.001 & 0.082 & -0.052 & -1.390 & -0.019 & -1.100 \\
\hline 0.75 & $0.036^{\mathrm{a}}$ & 11.542 & -0.011 & -1.139 & $-0.129^{a}$ & -8.877 & 0.009 & 0.466 & -0.036 & -1.039 & -0.009 & -0.571 \\
\hline 0.8 & $0.044^{\mathrm{a}}$ & 13.494 & -0.008 & -0.686 & $-0.126^{\mathrm{a}}$ & -8.336 & -0.006 & -0.332 & -0.057 & -1.566 & -0.009 & -0.647 \\
\hline 0.85 & $0.050^{\mathrm{a}}$ & 14.760 & -0.005 & -0.471 & $-0.131^{\mathrm{a}}$ & -8.214 & 0.007 & 0.353 & -0.057 & -1.604 & -0.017 & -1.241 \\
\hline 0.9 & $0.060^{\mathrm{a}}$ & 15.635 & -0.011 & -0.754 & $-0.123^{a}$ & -7.262 & -0.005 & -0.271 & 0.031 & 0.908 & 0.003 & 0.126 \\
\hline 0.95 & $0.074^{\mathrm{a}}$ & 16.398 & -0.008 & -0.495 & $-0.098^{\mathrm{a}}$ & -3.284 & -0.008 & -0.349 & 0.012 & 0.237 & -0.007 & -0.334 \\
\hline
\end{tabular}


Appendix 3. CLRM and QR models results including EPU (Geopolitical Acts)

Panel A. Global Travel \& Leisure Index

\begin{tabular}{|c|c|c|c|c|c|c|c|c|c|c|c|c|}
\hline & $\mathrm{c}$ & & GPR-ACTS & & VIX & & EPU & & OIL & & CRISIS & \\
\hline OLS & $0.007^{\mathrm{a}}$ & 3.273 & $-0.013^{\mathrm{a}}$ & -3.126 & $-0.111^{\mathrm{a}}$ & -9.555 & $-0.038^{\mathrm{a}}$ & -2.946 & -0.019 & -0.724 & $-0.031^{\mathrm{a}}$ & -3.596 \\
\hline 0.05 & $-0.062^{\mathrm{a}}$ & -6.752 & $-0.017^{\mathrm{a}}$ & -2.782 & $-0.135^{\mathrm{a}}$ & -4.148 & $-0.106^{\mathrm{a}}$ & -2.666 & -0.036 & -0.271 & -0.042 & -0.910 \\
\hline 0.1 & $-0.038^{a}$ & -7.662 & $-0.020^{\mathrm{b}}$ & -2.490 & $-0.155^{\mathrm{a}}$ & -5.590 & $-0.085^{\mathrm{a}}$ & -3.870 & 0.015 & 0.311 & -0.022 & -1.384 \\
\hline 0.15 & $-0.028^{a}$ & -4.982 & -0.016 & -1.583 & $-0.137^{a}$ & -4.176 & $-0.069^{\mathrm{a}}$ & -2.958 & -0.004 & -0.069 & -0.039 & -0.885 \\
\hline 0.2 & $-0.018^{a}$ & -5.426 & -0.008 & -1.390 & $-0.121^{\mathrm{a}}$ & -7.249 & $-0.058^{\mathrm{a}}$ & -2.982 & -0.010 & -0.254 & $-0.029^{\mathrm{b}}$ & -2.302 \\
\hline 0.25 & $-0.011^{\mathrm{a}}$ & -3.756 & $-0.010^{c}$ & -1.783 & $-0.123^{\mathrm{a}}$ & -7.812 & $-0.043^{b}$ & -2.427 & -0.037 & -1.000 & $-0.034^{b}$ & -2.431 \\
\hline 0.3 & $-0.007^{\mathrm{a}}$ & -2.615 & $-0.011^{\mathrm{b}}$ & -1.984 & $-0.121^{\mathrm{a}}$ & -7.571 & $-0.034^{\mathrm{b}}$ & -2.432 & -0.059 & -1.424 & $-0.025^{\mathrm{a}}$ & -2.768 \\
\hline 0.35 & -0.004 & -1.434 & $-0.009^{c}$ & -1.684 & $-0.124^{\mathrm{a}}$ & -7.783 & $-0.030^{\mathrm{b}}$ & -2.304 & $-0.066^{c}$ & -1.865 & $-0.027^{\mathrm{a}}$ & -2.831 \\
\hline 0.4 & 0.002 & 0.965 & -0.008 & -1.521 & $-0.117^{\mathrm{a}}$ & -7.784 & $-0.024^{\mathrm{b}}$ & -2.010 & $-0.064^{\mathrm{c}}$ & -1.935 & $-0.032^{\mathrm{a}}$ & -3.158 \\
\hline 0.45 & $0.005^{\mathrm{b}}$ & 2.090 & $-0.010^{c}$ & -1.962 & $-0.112^{\mathrm{a}}$ & -7.809 & $-0.026^{c}$ & -2.268 & $-0.064^{b}$ & -2.034 & $-0.030^{\mathrm{a}}$ & -2.949 \\
\hline 0.5 & $0.010^{\mathrm{a}}$ & 4.196 & $-0.013^{\mathrm{b}}$ & -2.472 & $-0.111^{\mathrm{a}}$ & -8.217 & $-0.021^{\mathrm{c}}$ & -1.752 & $-0.057^{\mathrm{c}}$ & -1.837 & $-0.031^{\mathrm{a}}$ & -3.045 \\
\hline 0.55 & $0.013^{\mathrm{a}}$ & 5.307 & $-0.015^{\mathrm{a}}$ & -2.776 & $-0.117^{\mathrm{a}}$ & -8.429 & $-0.020^{c}$ & -1.659 & $-0.055^{\mathrm{c}}$ & -1.800 & $-0.032^{\mathrm{a}}$ & -3.143 \\
\hline 0.6 & $0.017^{\mathrm{a}}$ & 7.079 & $-0.011^{\mathrm{c}}$ & -1.880 & $-0.109^{a}$ & -8.845 & -0.018 & -1.422 & $-0.054^{\mathrm{c}}$ & -1.801 & $-0.034^{\mathrm{a}}$ & -3.330 \\
\hline 0.65 & $0.021^{\mathrm{a}}$ & 8.719 & $-0.011^{\mathrm{c}}$ & -1.776 & $-0.109^{\mathrm{a}}$ & -8.669 & -0.020 & -1.408 & -0.025 & -0.806 & $-0.029^{b}$ & -2.477 \\
\hline 0.7 & $0.024^{\mathrm{a}}$ & 9.853 & -0.010 & -1.480 & $-0.101^{\mathrm{a}}$ & -8.130 & -0.019 & -1.147 & -0.037 & -1.224 & $-0.030^{\mathrm{b}}$ & -2.441 \\
\hline 0.75 & $0.028^{\mathrm{a}}$ & 11.042 & -0.008 & -1.249 & $-0.098^{a}$ & -7.632 & -0.020 & -1.106 & -0.014 & -0.439 & -0.027 & -1.589 \\
\hline 0.8 & $0.033^{\mathrm{a}}$ & 12.203 & -0.003 & -0.473 & $-0.099^{\mathrm{a}}$ & -8.143 & -0.025 & -1.378 & -0.001 & -0.041 & -0.028 & -1.912 \\
\hline 0.85 & $0.041^{\mathrm{a}}$ & 12.939 & -0.007 & -0.964 & $-0.090^{\mathrm{a}}$ & -5.156 & -0.025 & -1.339 & -0.016 & -0.583 & -0.018 & -1.265 \\
\hline 0.9 & $0.050^{\mathrm{a}}$ & 13.432 & -0.007 & -1.028 & $-0.086^{\mathrm{a}}$ & -4.310 & $-0.036^{c}$ & -1.699 & 0.001 & 0.033 & -0.011 & -0.665 \\
\hline 0.95 & $0.061^{\mathrm{a}}$ & 16.412 & -0.002 & -0.230 & $-0.070^{\mathrm{a}}$ & -3.604 & -0.022 & -0.875 & -0.001 & -0.014 & -0.015 & -0.899 \\
\hline \multicolumn{13}{|c|}{ Panel B. Asia-Pacific Travel \& Leisure Index } \\
\hline OLS & 0.002 & 0.792 & -0.004 & -0.812 & $-0.085^{\mathrm{a}}$ & -5.852 & $-0.036^{\mathrm{b}}$ & -2.257 & 0.006 & 0.175 & -0.016 & -1.474 \\
\hline 0.05 & $-0.083^{\mathrm{a}}$ & -8.082 & -0.011 & -0.389 & $-0.139^{b}$ & -2.319 & $-0.080^{c}$ & -1.751 & 0.080 & 0.327 & -0.030 & -0.550 \\
\hline 0.1 & $-0.057^{\mathrm{a}}$ & -9.463 & -0.005 & -0.345 & -0.054 & -1.545 & $-0.041^{b}$ & -2.409 & -0.013 & -0.161 & -0.007 & -0.285 \\
\hline 0.15 & $-0.040^{\mathrm{a}}$ & -8.508 & -0.012 & -1.271 & $-0.063^{b}$ & -2.493 & -0.018 & -0.663 & 0.011 & 0.167 & -0.011 & -0.676 \\
\hline 0.2 & $-0.031^{\mathrm{a}}$ & -6.909 & -0.009 & -1.129 & $-0.083^{a}$ & -3.000 & -0.031 & -0.930 & 0.022 & 0.389 & -0.010 & -0.728 \\
\hline 0.25 & $-0.020^{\mathrm{a}}$ & -5.587 & -0.007 & -0.971 & $-0.081^{\mathrm{a}}$ & -4.837 & -0.020 & -0.834 & 0.052 & 1.167 & -0.012 & -1.000 \\
\hline
\end{tabular}




\begin{tabular}{|c|c|c|c|c|c|c|c|c|c|c|c|c|}
\hline 0.3 & $-0.014^{\mathrm{a}}$ & -4.630 & -0.002 & -0.275 & $-0.086^{a}$ & -6.733 & -0.017 & -0.864 & 0.002 & 0.052 & -0.012 & -0.956 \\
\hline 0.35 & $-0.009^{\mathrm{a}}$ & -3.223 & -0.003 & -0.560 & $-0.093^{\mathrm{a}}$ & -7.693 & -0.026 & -1.353 & -0.009 & -0.227 & -0.012 & -0.901 \\
\hline 0.4 & $-0.005^{\mathrm{c}}$ & -1.655 & 0.002 & 0.323 & $-0.092^{\mathrm{a}}$ & -7.757 & -0.025 & -1.296 & 0.002 & 0.048 & -0.015 & -1.079 \\
\hline 0.45 & -0.001 & -0.250 & 0.000 & 0.077 & $-0.094^{\mathrm{a}}$ & -7.930 & -0.018 & -1.000 & 0.028 & 0.818 & -0.015 & -1.166 \\
\hline 0.5 & 0.003 & 1.243 & 0.001 & 0.163 & $-0.091^{a}$ & -7.490 & -0.024 & -1.317 & 0.033 & 0.911 & -0.012 & -0.970 \\
\hline 0.55 & $0.008^{a}$ & 2.780 & 0.002 & 0.385 & $-0.083^{a}$ & -6.161 & -0.021 & -1.215 & -0.002 & -0.058 & -0.011 & -0.955 \\
\hline 0.6 & $0.012^{\mathrm{a}}$ & 4.203 & 0.003 & 0.571 & $-0.083^{a}$ & -5.433 & -0.027 & -1.496 & -0.030 & -0.957 & -0.011 & -1.156 \\
\hline 0.65 & $0.017^{\mathrm{a}}$ & 5.902 & 0.006 & 1.096 & $-0.080^{\mathrm{a}}$ & -4.722 & -0.028 & -1.590 & -0.002 & -0.054 & -0.012 & -1.075 \\
\hline 0.7 & $0.023^{\mathrm{a}}$ & 7.694 & 0.006 & 0.968 & $-0.079^{\mathrm{a}}$ & -4.352 & -0.029 & -1.507 & 0.006 & 0.174 & -0.009 & -0.886 \\
\hline 0.75 & $0.028^{\mathrm{a}}$ & 8.825 & 0.001 & 0.184 & $-0.089^{a}$ & -3.943 & -0.026 & -1.345 & -0.008 & -0.215 & -0.012 & -1.317 \\
\hline 0.8 & $0.035^{\mathrm{a}}$ & 9.465 & 0.003 & 0.460 & $-0.089^{a}$ & -3.399 & -0.018 & -0.883 & -0.033 & -0.779 & $-0.018^{b}$ & -2.011 \\
\hline 0.85 & $0.043^{\mathrm{a}}$ & 10.855 & 0.004 & 0.484 & $-0.079^{a}$ & -2.871 & -0.010 & -0.433 & -0.039 & -1.060 & $-0.026^{\mathrm{a}}$ & -3.124 \\
\hline 0.9 & $0.056^{\mathrm{a}}$ & 11.469 & 0.010 & 0.984 & $-0.090^{\mathrm{a}}$ & -3.339 & -0.020 & -0.802 & -0.021 & -0.537 & $-0.033^{\mathrm{a}}$ & -3.241 \\
\hline 0.95 & $0.079^{\mathrm{a}}$ & 12.238 & -0.012 & -0.953 & $-0.082^{\mathrm{a}}$ & -2.738 & -0.001 & -0.020 & -0.009 & -0.268 & $-0.046^{\mathrm{a}}$ & -3.590 \\
\hline \multicolumn{13}{|c|}{ Panel C. Europe Travel \& Leisure Index } \\
\hline OLS & $0.007^{\mathrm{b}}$ & 2.587 & $-0.015^{\mathrm{a}}$ & -2.931 & $-0.111^{\mathrm{a}}$ & -7.766 & $-0.069^{\mathrm{a}}$ & -4.402 & $-0.083^{\mathrm{a}}$ & -2.616 & $-0.049^{\mathrm{a}}$ & -4.579 \\
\hline 0.05 & $-0.068^{\mathrm{a}}$ & -10.451 & $-0.014^{\mathrm{b}}$ & -2.048 & $-0.166^{\mathrm{a}}$ & -8.054 & $-0.134^{\mathrm{a}}$ & -4.909 & $-0.182^{b}$ & -1.995 & $-0.094^{\mathrm{b}}$ & -2.107 \\
\hline 0.1 & $-0.047^{\mathrm{a}}$ & -9.991 & $-0.015^{c}$ & -1.889 & $-0.137^{\mathrm{a}}$ & -5.808 & -0.120 & -7.375 & -0.017 & -0.378 & $-0.050^{\mathrm{a}}$ & -3.367 \\
\hline 0.15 & $-0.037^{\mathrm{a}}$ & -8.482 & -0.007 & -0.806 & $-0.125^{\mathrm{a}}$ & -6.278 & $-0.119^{a}$ & -6.355 & -0.053 & -1.096 & $-0.051^{\mathrm{a}}$ & -3.370 \\
\hline 0.2 & $-0.026^{\mathrm{a}}$ & -6.238 & -0.006 & -0.753 & $-0.117^{\mathrm{a}}$ & -6.356 & $-0.091^{\mathrm{a}}$ & -4.204 & -0.077 & -1.370 & $-0.047^{\mathrm{a}}$ & -3.442 \\
\hline 0.25 & $-0.018^{\mathrm{a}}$ & -4.824 & -0.009 & -1.172 & $-0.126^{a}$ & -6.812 & $-0.079^{a}$ & -3.924 & $-0.116^{\mathrm{a}}$ & -2.798 & $-0.055^{\mathrm{a}}$ & -4.459 \\
\hline 0.3 & $-0.012^{\mathrm{a}}$ & -3.326 & -0.009 & -1.218 & $-0.119^{a}$ & -6.914 & $-0.060^{a}$ & -2.969 & $-0.105^{\mathrm{b}}$ & -2.509 & $-0.057^{\mathrm{a}}$ & -4.102 \\
\hline 0.35 & $-0.008^{b}$ & -2.526 & -0.010 & -1.225 & $-0.111^{a}$ & -6.919 & $-0.054^{a}$ & -2.763 & $-0.106^{a}$ & -2.630 & $-0.056^{\mathrm{a}}$ & -3.961 \\
\hline 0.4 & 0.001 & 0.383 & -0.011 & -1.228 & $-0.111^{a}$ & -7.825 & $-0.039^{a}$ & -2.444 & $-0.099^{a}$ & -2.603 & $-0.062^{\mathrm{a}}$ & -4.372 \\
\hline 0.45 & $0.005^{\mathrm{a}}$ & 1.801 & -0.011 & -1.231 & $-0.110^{\mathrm{a}}$ & -7.607 & $-0.045^{a}$ & -2.838 & $-0.105^{a}$ & -2.865 & $-0.052^{\mathrm{a}}$ & -3.768 \\
\hline 0.5 & $0.008^{a}$ & 2.750 & $-0.012^{\mathrm{b}}$ & -2.135 & $-0.108^{a}$ & -7.540 & $-0.042^{a}$ & -2.716 & $-0.102^{a}$ & -2.805 & $-0.053^{\mathrm{a}}$ & -3.746 \\
\hline 0.55 & $0.012^{\mathrm{a}}$ & 4.180 & $-0.013^{\mathrm{b}}$ & -2.374 & $-0.101^{a}$ & -7.503 & $-0.037^{\mathrm{b}}$ & -2.305 & $-0.099^{a}$ & -2.818 & $-0.052^{\mathrm{a}}$ & -3.353 \\
\hline 0.6 & $0.019^{\mathrm{a}}$ & 6.182 & $-0.012^{\mathrm{b}}$ & -2.163 & $-0.102^{a}$ & -7.020 & -0.018 & -1.045 & $-0.080^{\mathrm{b}}$ & -2.296 & $-0.049^{\mathrm{a}}$ & -3.612 \\
\hline 0.65 & $0.022^{\mathrm{a}}$ & 7.201 & $-0.013^{\mathrm{b}}$ & -2.332 & $-0.094^{\mathrm{a}}$ & -6.402 & -0.025 & -1.428 & $-0.074^{b}$ & -2.131 & $-0.049^{\mathrm{a}}$ & -3.758 \\
\hline 0.7 & $0.027^{\mathrm{a}}$ & 8.742 & $-0.013^{\mathrm{b}}$ & -2.225 & $-0.092^{a}$ & -6.073 & -0.011 & -0.602 & -0.061 & -1.716 & $-0.049^{\mathrm{a}}$ & -3.949 \\
\hline
\end{tabular}




\begin{tabular}{|c|c|c|c|c|c|c|c|c|c|c|c|c|}
\hline 0.75 & $0.034^{\mathrm{a}}$ & 10.983 & $-0.013^{\mathrm{b}}$ & -2.124 & $-0.086^{a}$ & -4.480 & -0.009 & -0.480 & -0.057 & -1.486 & $-0.053^{a}$ & -4.646 \\
\hline 0.8 & $0.041^{\mathrm{a}}$ & 12.640 & -0.009 & -1.418 & $-0.063^{a}$ & -2.793 & -0.016 & -0.668 & -0.059 & -1.687 & $-0.056^{a}$ & -4.846 \\
\hline 0.85 & $0.048^{\mathrm{a}}$ & 13.952 & -0.008 & -0.971 & $-0.084^{a}$ & -2.803 & -0.030 & -0.818 & -0.077 & -1.586 & $-0.046^{\mathrm{b}}$ & -2.394 \\
\hline 0.9 & $0.057^{\mathrm{a}}$ & 15.604 & -0.008 & -0.741 & $-0.076^{\mathrm{b}}$ & -2.534 & -0.020 & -0.514 & -0.032 & -0.668 & $-0.043^{b}$ & -1.975 \\
\hline 0.95 & $0.075^{\mathrm{a}}$ & 9.097 & -0.003 & -0.211 & -0.025 & -0.386 & -0.030 & -0.378 & -0.073 & -0.825 & -0.029 & -0.982 \\
\hline \multicolumn{13}{|c|}{ Panel D. North America Travel \& Leisure Index } \\
\hline OLS & $0.009^{\mathrm{a}}$ & 3.842 & $-0.015^{\mathrm{a}}$ & -3.123 & $-0.141^{\mathrm{a}}$ & -10.578 & -0.010 & -0.708 & -0.039 & -1.300 & $-0.032^{\mathrm{a}}$ & -3.229 \\
\hline 0.05 & $-0.065^{a}$ & -10.975 & $-0.023^{\mathrm{a}}$ & -3.010 & $-0.158^{a}$ & -6.532 & $-0.056^{\mathrm{a}}$ & -2.953 & 0.049 & 0.686 & $-0.044^{b}$ & -2.024 \\
\hline 0.1 & $-0.042^{a}$ & -6.779 & $-0.016^{\mathrm{c}}$ & -1.632 & $-0.170^{a}$ & -4.543 & $-0.020^{\mathrm{b}}$ & -1.824 & -0.019 & -0.352 & $-0.046^{\mathrm{c}}$ & -1.821 \\
\hline 0.15 & $-0.031^{a}$ & -6.725 & $-0.014^{\mathrm{c}}$ & -1.688 & $-0.166^{\mathrm{a}}$ & -5.783 & -0.005 & -0.150 & -0.034 & -0.575 & $-0.062^{\mathrm{a}}$ & -3.009 \\
\hline 0.2 & $-0.025^{a}$ & -6.287 & -0.010 & -1.371 & $-0.160^{\mathrm{a}}$ & -6.159 & 0.006 & 0.231 & -0.071 & -1.498 & $-0.073^{\mathrm{a}}$ & -3.510 \\
\hline 0.25 & $-0.015^{\mathrm{a}}$ & -4.204 & $-0.014^{\mathrm{c}}$ & -1.719 & $-0.161^{\mathrm{a}}$ & -6.388 & -0.001 & -0.045 & $-0.123^{\mathrm{b}}$ & -2.540 & -0.068 & -1.631 \\
\hline 0.3 & $-0.007^{b}$ & -2.198 & -0.011 & -1.486 & $-0.153^{a}$ & -6.448 & -0.010 & -0.482 & -0.070 & -1.516 & -0.045 & -1.293 \\
\hline 0.35 & -0.002 & -0.690 & -0.010 & -1.426 & $-0.136^{\mathrm{a}}$ & -6.448 & -0.011 & -0.567 & -0.068 & -1.553 & -0.031 & -1.615 \\
\hline 0.4 & 0.004 & 1.248 & -0.010 & -1.514 & $-0.134^{\mathrm{a}}$ & -6.533 & -0.008 & -0.434 & $-0.068^{c}$ & -1.586 & -0.026 & -1.487 \\
\hline 0.45 & $0.009^{a}$ & 3.044 & -0.006 & -1.049 & $-0.131^{a}$ & -6.925 & -0.017 & -0.969 & $-0.088^{a}$ & -2.274 & -0.027 & -1.677 \\
\hline 0.5 & $0.011^{\mathrm{a}}$ & 3.748 & -0.007 & -1.121 & $-0.132^{a}$ & -7.501 & -0.012 & -0.725 & $-0.080^{a}$ & -2.265 & -0.014 & -0.850 \\
\hline 0.55 & $0.015^{\mathrm{a}}$ & 5.461 & -0.008 & -1.432 & $-0.132^{a}$ & -8.069 & -0.003 & -0.186 & $-0.083^{a}$ & -2.393 & -0.018 & -1.162 \\
\hline 0.6 & $0.020^{\mathrm{a}}$ & 7.042 & $-0.011^{\mathrm{b}}$ & -1.778 & $-0.133^{a}$ & -8.499 & 0.000 & -0.027 & $-0.077^{\mathrm{b}}$ & -2.221 & -0.024 & -1.528 \\
\hline 0.65 & $0.025^{\mathrm{a}}$ & 8.961 & $-0.009^{\mathrm{c}}$ & -1.694 & $-0.125^{\mathrm{a}}$ & -8.900 & -0.004 & -0.265 & $-0.069^{b}$ & -2.063 & -0.021 & -1.167 \\
\hline 0.7 & $0.030^{\mathrm{a}}$ & 10.299 & -0.008 & -1.554 & $-0.126^{a}$ & -8.986 & -0.011 & -0.590 & $-0.071^{\mathrm{b}}$ & -2.123 & -0.020 & -1.135 \\
\hline 0.75 & $0.037^{\mathrm{a}}$ & 11.844 & $-0.013^{b}$ & -2.121 & $-0.124^{\mathrm{a}}$ & -8.571 & 0.000 & -0.011 & -0.043 & -1.326 & -0.005 & -0.350 \\
\hline 0.8 & $0.042^{\mathrm{a}}$ & 13.172 & -0.010 & -1.470 & $-0.120^{a}$ & -8.298 & -0.002 & -0.112 & -0.025 & -0.726 & -0.006 & -0.442 \\
\hline 0.85 & $0.051^{\mathrm{a}}$ & 14.745 & -0.012 & -1.493 & $-0.129^{a}$ & -8.347 & -0.003 & -0.163 & -0.033 & -0.929 & -0.015 & -1.122 \\
\hline 0.9 & $0.060^{\mathrm{a}}$ & 16.021 & -0.010 & -1.032 & $-0.124^{a}$ & -7.672 & -0.010 & -0.506 & 0.022 & 0.622 & -0.004 & -0.260 \\
\hline 0.95 & $0.075^{\mathrm{a}}$ & 15.685 & -0.008 & -0.788 & $-0.101^{a}$ & -3.162 & 0.015 & 0.579 & 0.027 & 0.557 & -0.009 & -0.449 \\
\hline
\end{tabular}

Notes. (a), (b) and (c) denote the statistical significance at the $1 \%, 5 \%$ and $10 \%$ levels. 